\title{
Interdiffusion und Interreaktion in epitaktischen metallischen Schichtsystemen unter dem Einfluß diffusionsinduzierter Spannungen
}

\author{
Dissertation \\ zur Erlangung des Doktorgrades \\ der Mathematisch-Naturwissenschaftlichen Fakultäten \\ der Georg-August-Universität zu Göttingen
}

vorgelegt von

Frank Hartung aus Fulda

Göttingen 2000 
Hartung, Frank (fh@nanocube.de):

Interdiffusion und Interreaktion in epitaktischen metallischen Schichtsystemen unter dem Einfluß diffusionsinduzierter Spannungen / vorgelegt von Frank Hartung / Universität Göttingen / Dissertation, 2000

Elektronische Veröffentlichung:

Niedersächsische Staats- und Universitätsbibliothek Göttingen

URL: http://webdoc.sub.gwdg.de/diss/2000/hartung_frank/index.htm

D7

Referent: $\quad$ Prof. Dr. R. Kirchheim

Korreferent: PD Dr. M. Seibt

Tag der mündlichen Prüfung: $\quad$ 21.06.2000 


\section{Inhaltsverzeichnis}

$\begin{array}{ll}\text { 1. Einleitung } & 1\end{array}$

2. Untersuchte Systeme 4

2.1. Silber-Gold . . . . . . . . . . . . . . . . . . . . . . . 4

2.2. Kupfer-Gold . . . . . . . . . . . . . . . . . 5

$\begin{array}{lr}\text { 3. Experimentelle Methoden } & 8\end{array}$

3.1. Präparation und thermische Behandlung . . . . . . . . . 8

3.2. Methoden und Instrumente . . . . . . . . . . . . . . . . 10

3.2.1. Sekundärionenmassenspektrometrie . . . . . . . . . . . 10

3.2.2. Elektronenmikroskopie . . . . . . . . . . . . . 11

4. Quantitative Auswertung der chemischen Analysetechniken 14

4.1. Analytische Z-Kontrastabbildung . . . . . . . . . . . . . . . . . 14

4.1.1. Bildintensitäten der reinen Elemente . . . . . . . . . . 15

4.1.2. Inkohärenz der HCDF Abbildung . . . . . . . . . . . . 17

4.1.3. Quantitative Beschreibung der Bildintensitäten . . . . . 20

4.1.4. Anpassung eines Streuquerschnitts . . . . . . . . . . . . 24

4.1.5. Mehrkomponentige Systeme . . . . . . . . . . . . 27

4.1.6. Auswerteverfahren . . . . . . . . . . . . . . . . . . . 29

4.1.7. Genauigkeit der chemischen Analyse . . . . . . . . . . . 29

4.1.8. Diskussion . . . . . . . . . . . . . . . . . . . . 31

4.2. Sekundärneutralteilchenmassenspektrometrie . . . . . . . . . . 33

4.2.1. TRIM-Simulation . . . . . . . . . . . . . . . 34

4.2.2. Kalibrierung der Meßtiefe . . . . . . . . . . . . . 35

4.2.3. Konzentrationsbestimmung . . . . . . . . . . . 36

4.2.4. Einfluß von Grenzflächen . . . . . . . . . . . . . . . . . . 39

4.2.5. Diskussion . . . . . . . . . . . . . . . . . . . 40 
$\begin{array}{ll}\text { 5. Charakterisierung der Schichtsysteme } & 43\end{array}$

5.1. Silber/Gold . . . . . . . . . . . . . . . . . . . . 43

5.2. Kupfer/Gold . . . . . . . . . . . . . . . . . . . . 44

5.2.1. Mikroskopische Charakterisierung der Grenzfläche . . . . . 45

5.2.2. Epitaktisches Schichtwachstum bei großer Gitterfehlpassung 48

6. Interdiffusion 51

6.1. Silber/Gold . . . . . . . . . . . . . . . . . . . 51

6.1.1. Experimentelle Ergebnisse . . . . . . . . . . . 51

6.1.2. Volumendiffusion in einem Schichtpaket . . . . . . . . . 54

6.1.3. Einfluß der Korngrenzdiffusion . . . . . . . . . . . . . 56

6.2. Kupfer/Gold . . . . . . . . . . . . . . . . . . . . 59

6.2.1. Experimentelle Ergebnisse . . . . . . . . . . . . . . 59

6.2.2. Frühstadium der Interdiffusion . . . . . . . . . . . . . . 61

6.2.3. Charakterisierung der Defektstruktur . . . . . . . . 62

6.2.4. Modellbildung für die Frühstadien der Diffusion . . . . . . 69

6.2.5. Diffusionsinduzierte Neubildung des Gefüges . . . . . . . 75

6.2.6. Aktivierungsenergie und Bildungsenthalpie für die Neubildung des Gefüges . . . . . . . . . . . . . 80

6.2.7. Zusammenfassung des Reaktionsverlaufs . . . . . . . . . . 82

6.3. Diskussion und Vergleich mit anderen Experimenten . . . . . . . . 83

7. Interreaktion in Kupfer/Gold 85

7.1. Experimentelle Ergebnisse . . . . . . . . . . . . . 85

7.2. Ordnungseinstellung . . . . . . . . . . . . . . . 88

7.3. Phasenbildungsmodelle . . . . . . . . . . . . . . . . . . 89

7.4. Diskussion . . . . . . . . . . . . . . . . . . . . . . 90 90

$\begin{array}{lr}\text { 8. Zusammenfassung } & 92\end{array}$

A. SNMS-Eichungen zum System Silber-Gold 94

$\begin{array}{ll}\text { Literaturverzeichnis } & 95\end{array}$ 


\section{Einleitung}

Metallische Schichtsysteme sind aufgrund ihrer ungewöhnlichen physikalischen Eigenschaften (elastische, thermodynamische, elektronische, magnetische, supraleitende) und ihres technologischen Potentials Gegenstand intensiver Forschung. Nach der Herstellung befinden sich die Schichten in einem extremen Nichtgleichgewichtszustand. Für die technische Anwendung stellt sich daher die Frage nach der Stabilität der Schichtstrukturen. In einem elektronischen Bauelement führt eine Veränderung der Schichtstrukturen möglicherweise zum unerwünschten Versagen des Bauelements. Kennt man die Reaktionsverläufe in den verwendeten Schichtsystemen genau, so kann man daraus im Herstellungsprozeß eine Mikrostruktur mit optimierten Eigenschaften erzeugen. Für den Physiker bieten Schichtsysteme noch weitere Möglichkeiten fundamentale Eigenschaften von Festkörpern zu untersuchen. Man kann sehr niedrige Diffusionskoeffizienten ohne den Einfluß schneller Transportwege messen und ungewöhnliche Effekte aufgrund sehr steiler Konzentrationsgradienten und während der Diffusion entstehende Spannungen beobachten. Auch kann sich der Weg des Systems in ein thermodynamisches Gleichgewicht durch die hohen treibenden Kräfte sehr stark von dem bekannten Verlauf in massiven Materialien unterscheiden.

Die Reaktionen in einem Dünnschichtsystem hängen von zahlreichen Parametern ab. Zum einen spielen thermodynamische Aspekte eine Rolle. Die Bildung möglicher Phasen und der damit verbundene Energiegewinn beeinflussen die Reaktion. Zum anderen ist die Kinetik des Systems zu beachten. Die Geschwindigkeit der einzelnen Prozesse bestimmt den Reaktionsverlauf. Zusätzlich beeinflussen die Mikrostruktur, reaktionsinduzierte Spannungen und deren Relaxation die Reaktion.

Der Transport in dünnen Schichten kann zu einem Spannungszustand in den Schichten führen, wenn in der Probe Volumen transportiert wird. Im Fall eines Schichtsystems mit zwei Elementen mit jeweils einer Mobilität $M_{i}$ und einem partiellen Molvolumen $\bar{V}_{i}$ führt ein Unterschied des Produkts $M_{i} \bar{V}_{i}$ zu einem Volumentransport. Als Folge eines Diffusionsprozesses schrumpft die eine Schicht während die andere schwillt. Die so erzeugten Spannungen können zum Beispiel durch Prozesse wie plastische Verformung relaxiert werden. Ob die erzeugten Spannungen für den Reaktionsverlauf bedeutend sind, ergibt sich aus einem Vergleich der Diffusionsgeschwindigkeit mit der Relaxationszeit zum Abbau der Spannungen. Ab einer genügend großen Diffusionsweite wird die Diffusion so langsam, daß die entstehenden Spannungen jederzeit durch Relaxationsprozesse auf einen unbedeutenden Wert reduziert werden können. Ist die Diffusionsweite dagegen kurz, so erfolgt die Diffusion so schnell, daß die erzeugten Spannungen nicht relaxiert 


\section{Einleitung}

werden können. In diesem Fall können bedeutende Spannung enstehen, die die Diffusion selbst beeinflussen.

Die Diffusion unter der Berücksichtigung von elastischen Spannungen wurde bereits von Larché und Cahn $[58,59]$ in einem Kontinuumsmodell beschrieben. Zusätzlich zu den elastischen Effekten wird von Stephenson die plastische Verformung durch die aufgebauten Spannungen berücksichtigt [102]. Diese Beschreibung ist jedoch so aufwendig, daß eine Verknüpfung mit experimentellen Ergebnissen schwierig ist. In beiden Modellen wird von einem Gleichgewicht der Leerstellenkonzentration ausgegangen, d.h. entsprechende Quellen zum Ausgleich der Leerstellenkonzentration müssen in ausreichender Zahl im System vorhanden sein. Die Modellvoraussetzungen sind in amorphen Schichtpaketen erfüllt. So konnte eine Beschreibung der Interdiffusion in amorphen Nickel-Zirkon Schichten mit dem Modell von Stephenson erfolgreich durchgeführt werden [34]. Problematisch ist die Anwendung dieser Modelle auf die Diffusion in dünnen, kristallinen Schichtsystemen, da die Modellvoraussetzung nicht mehr gegeben sind. In diesem Fall beginnt die lokale Mikrostruktur eine wichtige Rolle zu spielen.

Ein zentrales Thema dieser Arbeit ist die Untersuchung des Einflusses von diffusionsinduzierten Spannungen auf die Diffusion in dünnen Schichten. Zur Untersuchung wurden die Modellsysteme Kupfer-Gold und Silber-Gold ausgewählt, deren Gitterfehlpassung sich sehr stark unterscheidet. Kupfer und Gold weisen einen Unterschied der Gitterparameter von etwa 12\% auf. Zusätzlich unterscheiden sich die einzelnen intrinsischen Diffusionskoeffizienten erheblich, so daß sich die Mobilität in den Schichten unterscheidet. Im Gegensatz dazu besitzen Silber und Gold praktisch das gleiche Atomvolumen, jedoch unterscheidet sich die Mobilität der beiden Elemente wie im Kupfer-Gold System. Zusätzlich werden die Ergebnisse mit denen der Interdiffusion in Silber-Palladium Schichtsystemen verglichen $[52,53,54,55]$. Dieses System ist voll mischbar, die intrinsischen Diffusionskoeffizienten unterscheiden sich sehr deutlich und die Gitterfehlpassung ist im Vergleich zum Kupfer-Gold System mit etwa 5\% nur halb so groß. Aufgrund der großen Gitterfehlpassung wird der stärkste Einfluß der Spannungen auf die Diffusion in System Kupfer-Gold erwartet.

Sofern im Phasendiagramm Mischphasen auftauchen, werden sich diese im Verlauf der Interreaktion bilden. An der Grenzfläche zweier Schichten jedoch findet die Phasenbildung nicht gleichzeitig, sondern in einer für das System typischen Reihenfolge statt. Deshalb ist es interessant neben der Interdiffusion auch die Phasenbildungssequenz im System Kupfer-Gold bei tieferen Temperaturen zu untersuchen. Zur Erklärung dieser Reihenfolge werden in der Literatur verschiedene Modelle diskutiert. Tu und Gösele [32] stellen in ihrem Modell kinetische Überlegungen in den Vordergrund. Aufgrund der Wachstumsgeschwindigkeit der einzelnen Phasen wird auf deren Stabilität und die Reihenfolge der Phasenbildung geschlossen. Erweiterungen dieses Modells schließen auch den Einfluß von Spannungen auf 


\section{Einleitung}

die Phasenbildungssequenz ein [46]. Die im Modell verwendeten Parameter sind jedoch experimentell sehr schwer zugänglich, so daß Aussagen über reale Schichtsysteme nur schwer möglich sind. Außerdem wird eine eindimensionale Schichtstruktur der Phasen gefordert, die nicht immer gegeben ist. In der empirischen Regel von Pretorius $[60,85,86]$ werden thermodynamische Argumente berücksichtigt. Aufgrund der effektiven Bildungsenthalpie $\Delta H^{\prime}=\Delta H^{\text {Phase }} \cdot c_{\text {Liquidus }} / c_{\text {Phase }}$ bei der Konzentration $c_{\text {Liquidus }}$ der niedrigsten Liquidustemperatur wird hier auf die Phasenbildungssequenz geschlossen. Die erste Phase, die gebildet wird, ist die mit der höchsten effektiven Bildungsenthalpie. Im Gegensatz zum Modell von Tu und Gösele sind die verwendeten Parameter experimentell gut zugänglich. Die Regel von Pretorius liefert für eine große Zahl von Systemen eine richtige Vorhersage der Phasensequenz. In keinem der Modelle wird jedoch der Einfluß der Mikrostruktur auf die Phasenbildung berücksichtigt. Im System Kupfer-Gold liefert das Modell von Pretorius ohne Modifikationen eine falsche Phasensequenz. Ein Ziel dieser Arbeit ist die Klärung der Gründe, die in diesem Fall zu einer Abweichung von der empirischen Regel von Pretorius führt. Weiter wird untersucht, ob das Modell von Tu und Gösele oder eine weiterentwickelte Variante im System Kupfer-Gold eine bessere Beschreibung der Phasenbildung erlaubt.

Zur Untersuchung dieser Fragestellungen ist es notwendig, hochauflösende chemische Analysemethoden einzusetzen. Im Rahmen dieser Arbeit wurde zu diesem Zweck die Z-Kontrast Elektronenmikroskopie, die weitwinkelgestreute Elektronen zur Abbildung verwendet, weiterentwickelt. Die Entwicklung einer auf Mehrfachstreuung von Elektronen basierenden Beschreibung der Bildintensitäten ermöglichte ein hochauflösende chemische Analyse in defekt- und verzerrungsreichen Gebieten durchzuführen. Diese konnte im folgenden direkt mit einer strukturellen Analyse kombiniert werden. Zusätzlich wurde die Auswertung der Meßergebnisse, die mit der Sekundärneutralteilchenmassenspektroskopie mit Lasernachionisierung (SNMS) gewonnen wurden, so weiterentwickelt, daß eine quantitative chemische Analyse der ausgewählten Schichtsysteme erstmals durchgeführt werden konnte.

Die vorliegende Arbeit gliedert sich in sieben Kapitel. Nachdem im folgenden Kapitel auf die untersuchten Systeme Kupfer-Gold und Silber-Gold eingegangen wird, folgt die Beschreibung der verwendeten experimentellen Methoden. Die chemische Charakterisierung der Proben auf einer Nanometerskala mit der ZKontrast Elektronenmikroskopie und die SNMS ist wesentlicher Bestandteil dieser Arbeit. Eine Beschreibung und Erweiterungen dieser Techniken wird in Kapitel 4 behandelt. In Kapitel 5 werden die für diese Arbeit hergestellten Schichten charakterisiert. Die Ergebnisse der Interdiffusionsexperimente mit den Kupfer/Gold und den Silber/Gold Schichten sind in Kapitel 6 dargestellt. Abschließend wird in Kapitel 7 die Interreaktion in Kupfer/Gold Schichten behandelt. Die Ergebnisse aus den Kapiteln 6 und 7 werden jeweils mit Modellen verglichen, um die verschiedenen ablaufenden Prozesse zu identifizieren. 


\section{Untersuchte Systeme}

Im folgenden sollen die bekannten grundlegenden Eigenschaften der betrachten Schichtsysteme dargelegt werden. Es wird auf die Thermodynamik, die Kinetik und die Ordnungseinstellung in den untersuchten Systemen eingegangen.

\subsection{Silber-Gold}

Die Elemente Silber und Gold sind oberhalb Raumtemperatur vollständig mischbar. Das Phasendiagramm weist keine intermetallischen Phasen auf [67]. Beide Elemente wie auch alle Mischkristalle haben eine kubisch-flächenzentrierte Struktur. In Silber findet man einen Gitterparameter a von $a_{C u}=0.40857 \mathrm{~nm}$, in Gold von $a_{A u}=0.4078 \mathrm{~nm}$ [80]. Damit liegt der Unterschied der Gitterparameter bei $0.2 \%$ und kann im Folgenden vernachlässigt werden.

Das System Silber-Gold eignet sich aufgrund seiner kompletten Mischbarkeit und der geringen Gitterfehlpassung sehr gut für die Untersuchung der Interdiffusion. Die Volumen- und die Korngrenzdiffusion sowie die Druckabhängigkeit der Diffusion sind in der Vergangenheit ausführlich untersucht worden [14,62,63]. Aufgrund der gut bekannten Diffusionsdaten wurden neue Verfahren, wie zum Beispiel die Bestimmung von Diffusionsdaten aus Messungen an dünnen Schichten, an diesem System getestet. Daher sind Diffusionsdaten bis zu sehr tiefen Temperaturen bekannt [99, 121].

Daneben eignet sich das System zur Untersuchung des Einflusses unterschiedlicher Mobilitäten auf das Diffusionsverhalten. Der Tracerdiffusionskoeffizient von Silber ist dabei im Durchschnitt bei hohen Temperaturen um den Faktor 2.5 größer als der von Gold. Durch die Erzeugung und Vernichtung von Leerstellen in der Diffusionszone werden die unterschiedlichen Beweglichkeiten der Elemente ausgeglichen. Diese nichtkonservativen Prozesse ändern das Volumen und erzeugen Spannungen in der Diffusionszone. In entsprechenden Experimenten, die sich bislang dieser Fragestellung widmeten, wurde dabei eine Diffusionszone in der Größenordnung von einigen zehn Mikrometern bis zu einem Millimeter bei Auslagerungstemperturen oberhalb der halben Schmelztemperatur untersucht $[10,17,104]$.

In Experimenten mit dünnen Schichten von wenigen Nanometern Dicke finden Yang et al. eine kurzreichweitige Ordnung an der Grenzfläche bei Auslagerungstemperaturen zwischen 140 und $230{ }^{\circ} \mathrm{C}$ [123]. So finden die Autoren eine Hemmung der Interdiffusion durch die eingestellte kurzreichweitige Ordnungsstruktur. Bei sehr tiefen Temperaturen werden 3 Ordnungsphasen mit den Strukturen $\mathrm{L}_{2}$ 


\section{Untersuchte Systeme}

für $\mathrm{Ag}_{25} \mathrm{Au}_{75}$ und $\mathrm{Ag}_{75} \mathrm{Au}_{25}$ und $\mathrm{L1}_{0}$ für $\mathrm{Ag}_{50} \mathrm{Au}_{50}$ mit kritischen Temperaturen zwischen 100 und $200 \mathrm{~K}$ gefunden [74,95]. Bei höheren Temperaturen findet sich in der Literatur jedoch kein Hinweis auf die Bildung von Ordnungsphasen. Bei sehr kleinen Abmessungen der Diffusionszone wurde der Einfluß der anderer Effekten wie zum Beispiel Gradientenenergien auf die Diffusion sichtbar [14,123]. Da diese Effekte nur bei Diffusionswegen von wenigen Atomabständen eine Rolle spielen, können sie in dieser Arbeit aufgrund der vergleichsweise langen Diffusionswege vernachlässigt werden.

Die einfache Struktur und das Fehlen einer Gitterfehlpassung waren die Gründe für die Auswahl des Systems Silber-Gold. In dieser Arbeit wird darauf eingegangen, welchen Einfluß die unterschiedlichen Mobilitäten bei den untersuchten dünnen Schichten und niedrigen Temperaturen auf den Diffusionsverlauf haben. Die Auslagerungstemperatur wurde mit $350{ }^{\circ} \mathrm{C}$ aufgrund der bekannten Diffusionsdaten so gewählt, daß nach 10 Minuten eine wenige Nanometer breite Diffusionszone entsteht. Es wird auch untersucht, ob die von Yang et al. gefundene kurzreichweitige Ordnung die Diffusion in $100 \mathrm{~nm}$ dicken Schichten behindert.

\subsection{Kupfer-Gold}

Abbildung 2.1 zeigt einen Auschnitt aus dem Kupfer-Gold Phasendiagramm [67]. Unterhalb von $410{ }^{\circ} \mathrm{C}$ findet man drei intermetallische Ordnungsphasen mit einem breiten Existenzgebiet, oberhalb dieser Temperatur wird über das gesamte

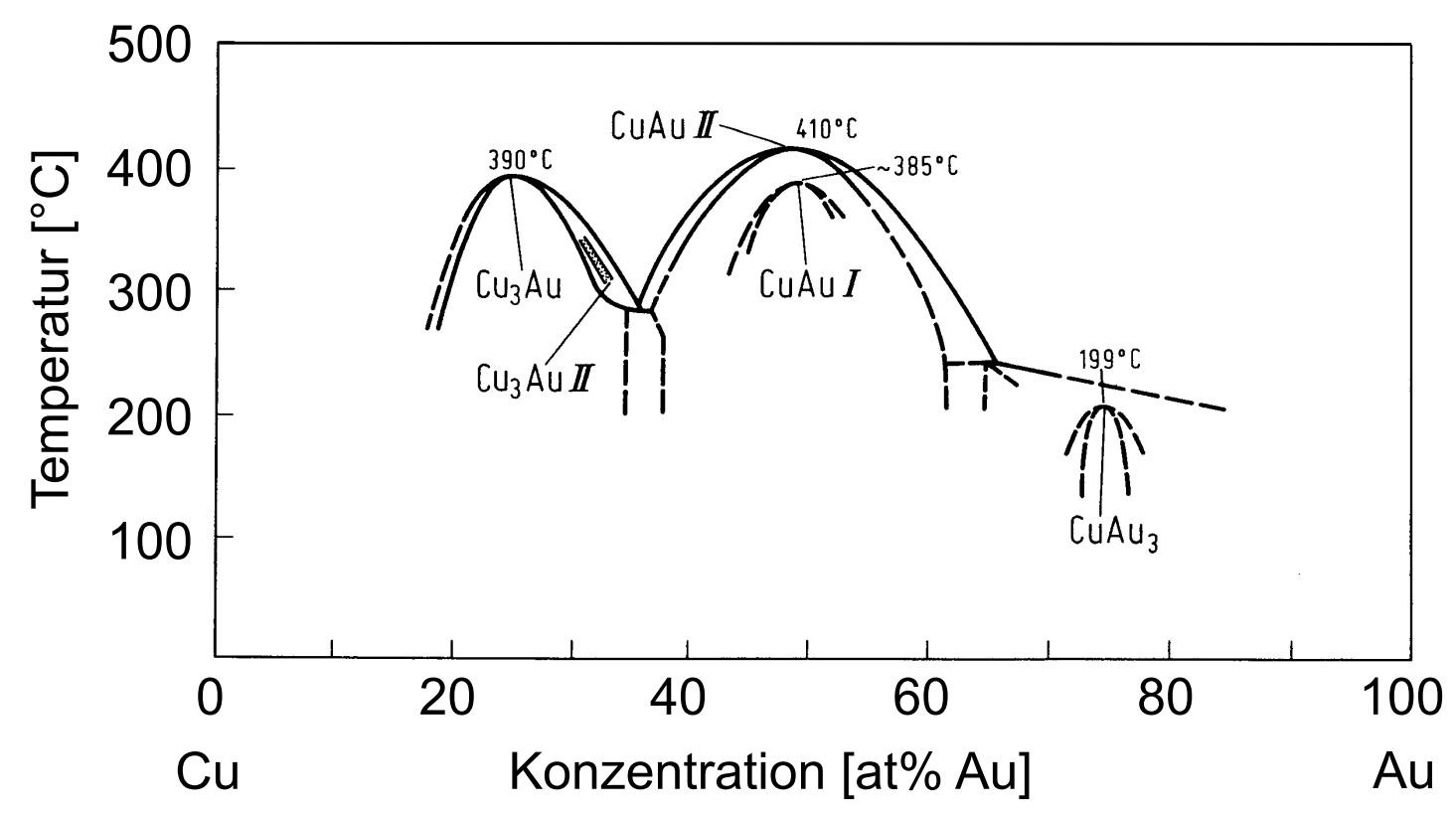

Abb. 2.1. Auschnitt des Kupfer-Gold Phasendiagramm nach Massalski [67]. 


\section{Untersuchte Systeme}

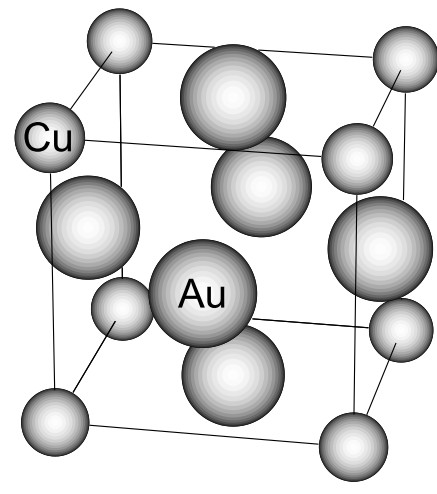

(a) $\mathrm{CuAu}_{3}-\mathrm{L}_{2}$

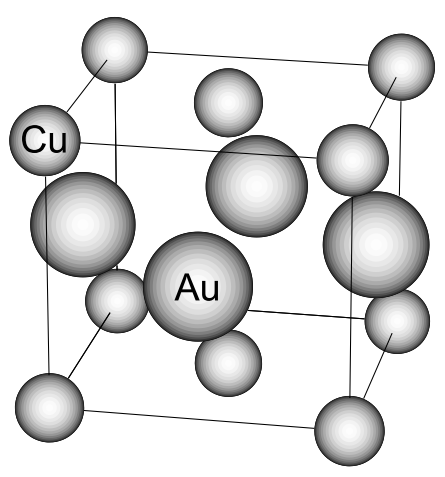

(b) $\mathrm{CuAu}-\mathrm{L} 1_{0}$

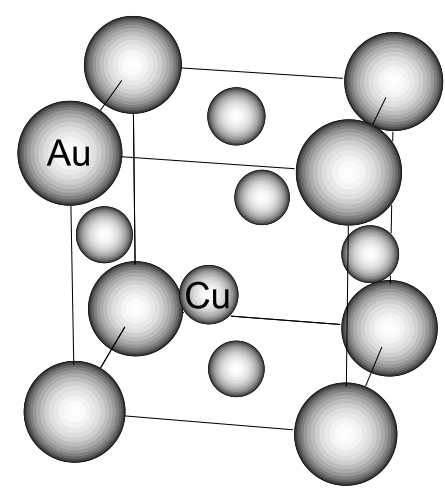

(c) $\mathrm{Cu}_{3} \mathrm{Au}-\mathrm{L}_{2}$

Abb. 2.2. Ordnungstrukturen des $\mathrm{Cu}-\mathrm{Au}$-Systems.

Konzentrationsgebiet ein kubisch-flächenzentrierter Mischkristall gebildet. Der Gitterparameter von Kupfer beträgt $0.36146 \mathrm{~nm}$, der von Gold 0.4078 nm [80]. Damit ergibt sich eine große Gitterfehlpassung von $12 \%$.

Bei dem System Kupfer-Gold handelt es sich um ein schwach ordnendes System, da der Ordnungsübergang weit unterhalb des Schmelzpunktes stattfindet. Die auftretenden Ordnungsphasen sind in Abbildung 2.2 zusammengestellt. Obwohl in einigen Arbeiten untersucht $[6,67,75,76]$, ist die Struktur der geordneten goldreichen Phase noch nicht sicher bestimmt. Die Annahme, daß die Phase $\mathrm{CuAu}_{3}$ eine $\mathrm{L}_{2}$ Struktur hat, konnte in Experimenten aufgrund der niedrigen Beweglichkeit bei den erforderlichen niedrigen Temperaturen und der noch stark vorhandenen Unordnung nicht eindeutig nachgewiesen werden. Neue First-Principle Untersuchungen ergeben sogar, daß eine $\mathrm{L}_{2}$ Struktur der $\mathrm{CuAu}_{3}$ Phase nicht stabil ist [79]. Man findet auf der goldreichen Seite im Grundzustand verschiedene komplexe Strukturen, die sich in ihrer Energie nur wenig unterscheiden. Dazu gehört zum Beispiel die $\beta 2\left(\mathrm{CuAu}_{2}\right)$ Phase. Die niedrigste in dieser Arbeit untersuchte Auslagerungstemperatur für die Phasenbildung liegt mit $230{ }^{\circ} \mathrm{C}$ nur wenig unter der Ordnungstemperatur von $240{ }^{\circ} \mathrm{C}$ [67] für die goldreiche Phase. Neben der langreichweitigen Ordnung ist auch die kurzreichweitige Ordnung von Bedeutung. Cowley gibt an, daß der Energiegewinn der geordneten Struktur aus zweitnächsten Nachbarn nur noch 10\% der der nächsten Nachbarn ausmacht [15]. Eine Nahordnung wurde später im System Kupfer-Gold in mehreren Arbeiten nachgewiesen $[6,15,72,88]$. Durch theoretischen Rechnungen wurde eine energetische Bevorzugung von ordnungseinstellenden Fluktuationen gefunden [120]. Ein Einfluß der Nahordnung auf die Diffusion in dünnen Schichten, wie er schon im Silber-Gold System berichtet wurde, ist damit nicht auszuschließen. In dieser Arbeit wird die niedrige Ordnungstemperatur ausgenutzt, um oberhalb der 


\section{Untersuchte Systeme}

Ordnungstemperatur unabhängig von der Ordnungseinstellung die Diffusion zu untersuchen und die gewonnenen Erkenntnisse bei der Untersuchung der Phasenbildung anzuwenden.

Schon 1939 wurden ersten Untersuchung zur Diffusion in Kupfer-Gold Schichtsystemen von DuMond et al. durchgeführt [20]. Später wurde unter anderem von Martin [66] festgestellt, daß die Diffusion bei niedrigen Temperaturen schneller ist, als man dies nach den Hochtemperaturdaten erwarten kann. Dieses Verhalten wird durch eine schnelle Korngrenzdiffusion erklärt. Dazu stellt Borders [9] fest, daß Kupfer schneller als Gold durch die Korngrenzen diffundiert und daß beide Elemente an der Korngrenze angereichert werden können. Aleshin [1] findet ein vom Bulkverhalten stark abweichendes Verhalten für Kupfer/Gold Dünnschichtsysteme. Die angegebenen Aktivierungsenergien für die Korngrenz- und Volumendiffusion liegen demnach sehr nahe beieinander. Aleshin stellt fest, daß die Ergebnisse durch hohe Defektdichten in den dünnen Schichten beeinflußt werden. Bei Experimenten mit extrem dünnen Schichten, in denen die Diffusionswege nur wenige Atomabstände betrugen, findet man auch im System Kupfer-Gold eine nichtlineares Diffusionsverhalten [71].

Im System Kupfer-Gold können Spannungen aufgrund von unterschiedlichen Mobilitäten der einzelnen Komponenten und dem stark unterschiedlichen Atomvolumen auftreten. So finden zum Beispiel Austin et. al. in Experimenten zur Korngrenzdiffusion an der Oberfläche in der Nähe der Korngrenzen Erhebungen [2]. Diese Erhebungen kompensieren die durch Volumendiffusion von der Korngrenze in das angrenzende Korn entstehenden Spannungen. Bei den auftretenden Spannungen spielen die elastischen Eigenschaften der Materialien eine wichtige Rolle. Aus den elastischen Konstanten der reinen Elemente ergibt sich, daß Kupfer $\left(\mathrm{E}=1.1 \cdot 10^{11} \mathrm{~N} / \mathrm{m}^{2}, \nu=0.356\right.$ bei $\left.700 \mathrm{~K}\right)$ härter als Gold $\left(\mathrm{E}=0.698 \cdot 10^{11} \mathrm{~N} / \mathrm{m}^{2}\right.$, $\nu=0.428$ bei $700 \mathrm{~K})$ ist [98]. Für die Mischkonzentrationen beschreibt eine lineare Näherung der elastischen Konstanten das Verhalten gut.

Während in späteren Interreaktionsstadien in massiven Proben grundsätzlich alle nach dem Gleichgewichtsphasendiagramm möglichen Phasen beobachtet werden, bildet sich in dünnen Schichten zu Beginn oft nur eine der nach dem Phasendiagramm möglichen Mischphasen. In Röntgenbeugungsexperimenten wird im System Kupfer-Gold zuerst die $\mathrm{Cu}_{3} \mathrm{Au}$ Phase gefunden, gefolgt von der $\mathrm{CuAu}_{3}$ Phase $[85,113]$. Erst sehr viel später findet man die $\mathrm{CuAu}$ Phase. Bei RBS-Messungen wird dagegen ein kontinuierlicher Konzentrationsverlauf gefunden, aus dem nicht direkt auf die Existenz einer neu gebildeten Phase geschlossen werden kann [9]. Auffallend ist auch die Veränderung der Mikrostruktur während der Phasenbildung. Mehrere Autoren finden in Röntgenbeugungsuntersuchungen eine Abnahme der Korngröße [9,113]. Aus jüngeren Experimenten gibt es konkrete Hinweise auf die Bildung eines neuen Korngefüges während der Interreaktion [38]. 


\section{Experimentelle Methoden}

Im Rahmen dieser Arbeit wurden Schichtsysteme aus den in Kapitel 2 aufgeführten Elementen präpariert und charakterisiert. Die experimentellen Details der Herstellung und die verwendeten Geräte sollen im folgenden beschrieben werden.

\subsection{Präparation und thermische Behandlung}

Die Schichtsysteme wurden auf verschiedene Substrate deponiert. Für die elektronenmikroskopischen Untersuchungen und die SNMS-Messungen wurden einkristalline, metallische Substrate verwendet, die selbst Teil der untersuchten Reaktionspaare waren. Im Fall von Kupfer-Gold wurden Kupfersubstrate, im Fall von Silber-Gold Silbersubstrate verwendet. Die Substrate wurden mittels Funkenerosion geschnitten. Für die Elektronenmikroskopie wurden sie zuerst mit dem Laueverfahren bezüglich ihrer Orientierung charakterisiert und so geschnitten, daß sie in der Querschnittspräparation in (110) Orientierung zum Elektronenstrahl lagen. Anschließend wurden die Substrate vorsichtig mechanisch poliert. Die Kupfersubstrate wurden elektrolytisch in einer verdünnten Phosphorsäure (2 Teile $\mathrm{H}_{2} \mathrm{O}, 3$ Teile $\mathrm{H}_{3} \mathrm{PO}_{4}$ ) bei 10 Volt poliert. Die Silbersubstrate wurden in einer Lösung aus $25 \mathrm{ml}$ destilliertem Wasser, $15 \mathrm{ml} \mathrm{H}_{2} \mathrm{O}_{2}$ und $60 \mathrm{ml}$ Ammoniaklösung bei $25^{\circ} \mathrm{C}$ poliert. Da die Herstellung einkristalliner, metallischer Substrate mit einem hohen Aufwand verbunden ist, wurden für die Röntgendiffraktometrie Glassubstrate verwendet. Dadurch wird auch das Problem umgangen, daß das Röntgenspektrum des metallischen Substrats das der Schichten überlagert. Durch Probemessungen wurde sichergestellt, daß die Glassubstrate selbst im Röntgenspektrum ein definiertes Untergrundspektrum von nur geringer Intensität liefern.

Die Schichtpakete wurden in verschiedenen Sputteranlagen hergestellt. Die Substrate wurden vor der Beschichtung mit einem Ionenstrahl gereinigt. Die Umschaltzeit zwischen den verschiedenen Targets betrug wenige Sekunden. Bei den elektronenmikroskopischen Untersuchungen und Messungen mit der SNMS konnten an den Grenzflächen keine Verunreinigungen nachgewiesen werden. Die Daten der Anlagen und der Beschichtungsparameter für die hergestellten Schichtsysteme sind in Tabelle 3.1 zusammengefaßt.

Die Auslagerungen der Proben erfolgte in einem Salzbadofen. Die Temperatur des Salzbads wurde mit einem Thermoelement direkt am Ort der ausgelagerten Probe gemessen. Dadurch kann die Temperatur bis auf $2{ }^{\circ} \mathrm{C}$ genau eingestellt werden. Nach der Auslagerungszeit wurden die Proben in einem Wasserbad abgeschreckt. 


\section{Experimentelle Methoden}

Tab. 3.1. Eigenschaften der verwendeten Beschichtungsanlagen und die dort hergestellten Schichtpakete.

\begin{tabular}{|c|c|c|}
\hline Schichten & $\begin{array}{c}\text { Rate } \\
{\left[\frac{\mathrm{nm}}{\mathrm{min}}\right]}\end{array}$ & $\begin{array}{c}\text { Restgasdruck } \\
{[\mathrm{mbar}]}\end{array}$ \\
\hline \hline $\mathrm{Cu}(50 \mathrm{~nm}) / \mathrm{Au}(50 \mathrm{~nm}) / \mathrm{Cu}(\mathrm{TEM})$ & 10 & $<1 \cdot 10^{-6}$ \\
\hline $\mathrm{Cu}(150 \mathrm{~nm}) / \mathrm{Au}(150 \mathrm{~nm}) / \mathrm{Cu}(\mathrm{TEM})$ & 20 & $<1 \cdot 10^{-8}$ \\
$\mathrm{Au}(200 \mathrm{~nm}) / \mathrm{Cu}(\mathrm{TEM})$ & & \\
{$[\mathrm{Cu}(25 \mathrm{~nm}) / \mathrm{Au}(25 \mathrm{~nm})]_{50} / \mathrm{Glas}(\mathrm{DSC})$} & & \\
{$[\mathrm{Cu}(50 \mathrm{~nm}) / \mathrm{Au}(50 \mathrm{~nm})]_{25} / \mathrm{Glas}(\mathrm{DSC})$} & & \\
$\mathrm{Au}(200 \mathrm{~nm}) /[\mathrm{Ag}(200 \mathrm{~nm}) / \mathrm{Au}(200 \mathrm{~nm})]_{2} / \mathrm{Ag}(\mathrm{TEM})$ & & \\
\hline $\mathrm{Cu}(150 \mathrm{~nm}) / \mathrm{Au}(150 \mathrm{~nm}) / \mathrm{Cu}(\mathrm{TEM})$ & 20 & $<1 \cdot 10^{-7}$ \\
$\mathrm{Au}(60 \mathrm{~nm}) / \mathrm{Cu}(60 \mathrm{~nm}) / \mathrm{Au}(60 \mathrm{~nm}) / \mathrm{Cu}(\mathrm{SNMS})$ & & \\
$\mathrm{Au}(60 \mathrm{~nm}) /[\mathrm{Cu}(60 \mathrm{~nm}) / \mathrm{Au}(60 \mathrm{~nm})]_{2} / \mathrm{Cu}(\mathrm{SNMS})$ & & \\
$\mathrm{Au}(100 \mathrm{~nm}) /[\mathrm{Ag}(100 \mathrm{~nm}) / \mathrm{Au}(100 \mathrm{~nm})]_{2} / \mathrm{Ag}(\mathrm{SNMS})$ & & \\
\hline
\end{tabular}

Die elektronenmikroskopischen Proben, die schon für die Querschnittspräparation vorbereitet waren, wurden ohne Schutzhülle in das Salzbad getaucht. Dadurch konnten die teilweise notwendigen, kurzen Auslagerungszeiten ohne eine Zeitkorrektur erreicht werden. In diesem Fall bewegen sich die Aufheiz- und Abkühlzeiten im Millisekundenbereich, so daß der Transport der Probe ins Wasserbad den zeitbegrenzenden Schritt darstellt. Es ließen sich Zeiten von wenigen Sekunden realisieren. In einem späteren Präparationsschritt wurde die vom Salz beeinflußte Schicht entfernt, so daß Beeinträchtigungen der Probe durch das Salz auszuschließen sind. Die Querschnittspräparation der elektronenmikroskopischen Proben wurden mit einer Gatan Duomill 600 Ionendünnungsanlage durchgeführt. Weitere Einzelheiten zu den verschiedenen Präparationsschritten sind in $[38,89]$ aufgeführt.

Bei allen anderen Proben für die SNMS-Messungen und Röntgenbeugungsuntersuchungen wurde durch geeignete Schutzhüllen eine Beeinträchtigung der Schichten durch das Salzbad verhindert. In einem Fall wurde eine Kupfer- und Aluminiumfolie verwendet, im anderen eine Tauchglocke aus Kupfer. Auch wurde bei der Herstellung der Schichten für diese Untersuchungen darauf geachtet, daß das Schichtpaket mit einer Golddecklage endet. Diese schützt das Schichtpaket während der Auslagerung. Durch Vergleich mit den Ergebnissen der querschnittspräparierten Proben wurde eine Korrektur für die Auslagerungszeiten aufgrund der schlechteren Wärmeankoppelung bestimmt. 


\section{Experimentelle Methoden}

\subsection{Methoden und Instrumente}

Für Struktur- und Texturmessungen wurde ein Vierkreisdiffraktometer X-Pert von Philips verwendet. Das Gerät ist mit einer Kobaltröntgenröhre ausgestattet. Zur Messung werden die beiden Linien $k_{\alpha 1}=0.178897 \mathrm{~nm}$ und $k_{\alpha 2}=0.179285$ $\mathrm{nm}$ verwendet, die im Intensitätsverhältniss $1 \mathrm{zu} 1$ auftreten. Weitere Experimente wurden an einem Zweikreisdiffraktometer mit einer Kupferröhre durchgeführt. In diesem Fall wurden die beiden Linien $k_{\alpha 1}=0.154051 \mathrm{~nm}$ und $k_{\alpha 2}=0.154433$ nm verwendet, die im Verhältnis $2 \mathrm{zu} 1$ auftreten. Die für die Strukturuntersuchung verwendeten Glassubstrate lieferten ein intensitätsschwaches, amorphes Untergrundspektrum, daß bei der Interpretation vernachlässigt werden kann.

Die Umwandlungswärme wurde mit einem Differential Scanning Calorimeter (DSC) Modell 7 der Firma Perkin Elmer bestimmt. Vor der Untersuchung wurde eine sorgfältige Eichung des Geräts für alle verwendeten Heizraten durchgeführt. Für die Untersuchungen mußten freistehende Schichtpakete von mindestens $2 \mathrm{mg}$ Gesamtgewicht hergestellt werden. Dazu wurde auf einen Glasträger eine 200 nm dicke, wasserlösliche Schicht aufgedampft. Auf die so vorbereiteten Substrate wurde im folgenden Präparationsschritt das metallische Schichtpaket aufgebracht. Das vom Substrat abgelöste Schichtpaket konnte anschließend im DSC untersucht werden.

\subsubsection{Sekundärionenmassenspektrometrie}

Als eine chemische Analysemethode wurde die Sekundärionenmassenspektrometrie (SIMS) eingesetzt. Dazu wird im allgemeinen eine Probe mittels Ionenbestrahlung abgetragen und die dabei entstehenden Ionen werden analysiert. Die für diese Arbeit verwendete Anlage ist schematisch in Abbildung 3.1 dargestellt. Zum Abtrag der Probe wurde die Argonionenquelle verwendet, die mit Beschleunigungsspannungen zwischen 0.7 und $10 \mathrm{kV}$ betrieben werden kann. Zwischen dem Abtrag durch die Argonionenquelle wird mit einer gepulsten Cäsium- oder Galliumionenquelle die aktuelle Oberfläche abgetragen und die entstehenden Ionen in einem Flugzeitmassenspektrometer analysiert. Als Startsignal für die Flugzeitmessung wird der Puls der Ionenquelle verwendet. Bei jedem Puls wird das gesamte Massenspektrum aufgezeichnet. Für die zu analysierenden Ionen werden Zeitfenster entsprechend ihrer Flugzeit gesetzt. Die Massenauflösung $m / \Delta m$ des Geräts hängt von der Pulslänge ab und beträgt unter normalen Bedingungen über 5000. Die Cäsiumionenquelle kann mit Beschleunigungsspannungen zwischen 0.7 und $10 \mathrm{kV}$, die Galliumionenquelle mit Beschleunigungsspannungen zwischen 13 und $25 \mathrm{kV}$ betrieben werden. Ein minimaler Strahldurchmesser von $100 \mathrm{~nm}$, der die laterale Aufösung des Geräts bestimmt, kann mit der Galliumquelle erreicht 


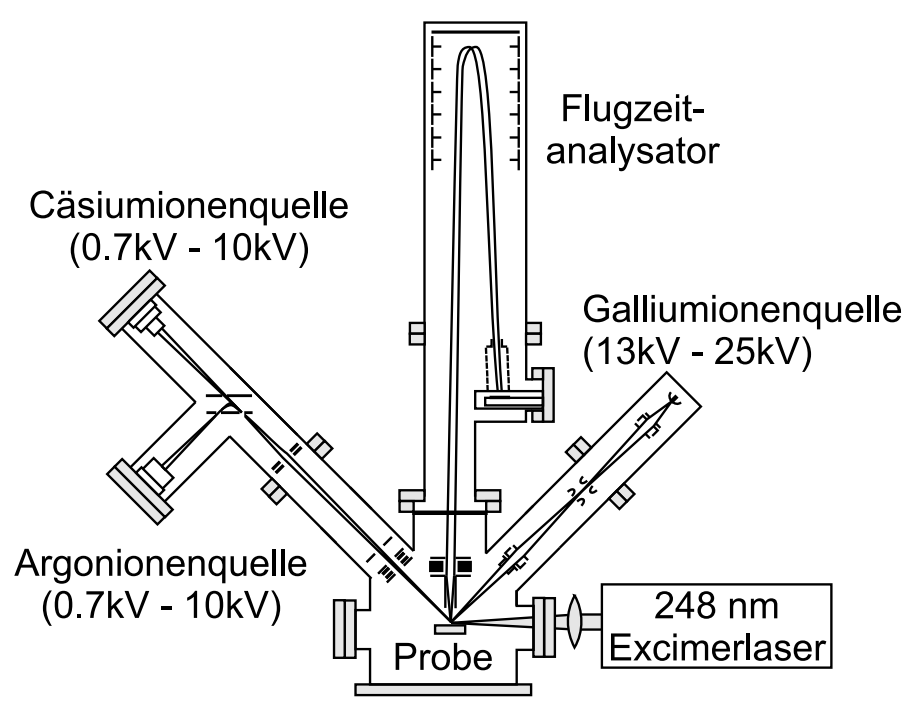

\begin{abstract}
Abb. 3.1. Schematische Darstellung des SIMS bzw. SNMS mit Lasernachionisierung. In dieser Arbeit wurde die Argon Ionenquelle für den Schichtabtrag und die Gallium Ionenquelle zur Analyse verwendet. Bei dem Laser zur Nachionisation handelt es sich um einen $248 \mathrm{~nm}$ Excimerlaser.
\end{abstract}

werden. In dieser Meßkonfiguration beträgt die Ionenausbeute jedoch nur wenige Prozent und ist von den zu analysierenden Elementen abhängig. Zusätzlich erschweren Matrixeffekte die quantitative Interpretation, wobei eine kleine Konzentrationsänderung eine starke Änderung der Ionenausbeute verursacht.

Daher wurde das Gerät mit einer Lasernachionisation erstmals eingesetzt. In dieser Konfiguration wird weiterhin Material mit einer gepulsten Analyseionenquelle von der Oberfläche abgetragen. Die entstehenden Ionen verlassen sehr schnell durch elektrostatische Abstoßung die oberflächennahen Bereiche. Die verbleibenden Neutralteilchen werden mit einem kurzen Laserpuls ionisiert und mit dem Flugzeitmassenspektrometer analysiert. Die Massenauflösung $m / \Delta m$ des Geräts beträgt in diesem Modus über 1000. Der Laserpuls dient nun als Startsignal für die Flugzeitmessung. An der verwendeten Anlage wird ein Excimerlaser mit einer Wellenlänge von $248 \mathrm{~nm}$ eingesetzt. Die zeitliche Abfolge ist so gewählt, daß nur nachionisierte Teilchen ausgewertet werden. Matrixeffekte sind in dieser Anordnung vernachlässigbar, da diese den Anteil der Neutralteilchen kaum verändern. Bei dieser Meßkonfiguration handelt es sich damit um eine Sekundärneutralteilchenmassenspektroskopie mit Lasernachionisierung (SNMS). Die für diese Arbeit für die verschiedenen Systeme verwendeten Analyseparameter sind in Tabelle 3.2 zusammengefaßt.

\subsubsection{Elektronenmikroskopie}

Für die Arbeiten standen ein Philips EM 420 ST Transmissionselektronenmikroskop mit $120 \mathrm{kV}$ Beschleunigungsspannung und ein Philips CM 200 FEG Transmissionselektronenmikroskop mit $200 \mathrm{kV}$ Beschleunigungsspannung zur Verfügung. Die Strukturanalyse mittels konventioneller Hellfeldabbildung wurde 


\section{Experimentelle Methoden}

Tab. 3.2. Zusammenstellung der Analyseparameter der SNMS.

\begin{tabular}{|c|c|c|c|}
\hline System & $\begin{array}{c}\text { Argonquelle } \\
\text { Dosis } \\
\text { Fläche }\end{array}$ & $\begin{array}{c}\text { Galliumquelle } \\
\text { Dosis } \\
\text { Fläche }\end{array}$ & $\begin{array}{c}\text { analysierte } \\
\text { Ionen }\end{array}$ \\
\hline $\mathrm{Cu}-\mathrm{Au}$ & $0.7 \mathrm{kV} / 8 \mathrm{nA}$ & $25 \mathrm{kV} / 0.01 \mathrm{pA}$ & ${ }^{197} \mathrm{Au},{ }^{63} \mathrm{Cu}$ \\
& $1 \cdot 10^{18}$ Ionen $/ \mathrm{cm}^{2}$ & $1 \cdot 10^{12}$ Ionen $/ \mathrm{cm}^{2}$ & ${ }^{65} \mathrm{Cu}$ \\
& $300 \times 300 \mu m^{2}$ & $78 \times 78 \mu m^{2}$ & \\
\hline $\mathrm{Ag}-\mathrm{Au}$ & $0.7 \mathrm{kV} / 8 \mathrm{nA}$ & $25 \mathrm{kV} / 0.01 \mathrm{pA}$ & ${ }^{197} \mathrm{Au},{ }^{107} \mathrm{Ag}$, \\
& $1 \cdot 10^{18}$ Ionen $/ \mathrm{cm}^{2}$ & $1 \cdot 10^{12}$ Ionen $/ \mathrm{cm}^{2}$ & ${ }^{109} \mathrm{Ag},{ }^{107} \mathrm{Ag}_{2}$ \\
& $300 \times 300 \mu m^{2}$ & $78 \times 78 \mu m^{2}$ & ${ }^{107} \mathrm{Ag}{ }^{109} \mathrm{Ag},{ }^{109} \mathrm{Ag}_{2}$ \\
\hline
\end{tabular}

Tab. 3.3. Daten der verwendeten Transmissionselektronenmikroskope.

\begin{tabular}{|l|l|l|}
\hline & Philips EM 420 ST & Philips CM 200 FEG \\
\hline Kathode & $\mathrm{LaB}_{6}$ Kathode & Feldemissionskathode \\
Beschleunigungsspannung U & $120 \mathrm{kV}$ & $200 \mathrm{kV}$ \\
Wellenlänge $\lambda$ & $3.349 \mathrm{pm}$ & $2.508 \mathrm{pm}$ \\
Sphärische Aberation $c_{s}$ & $1.35 \mathrm{~mm}$ & $0.49 \mathrm{~mm}$ \\
Punktauflösungsvermögen $\delta \mathrm{x}$ & $0.33 \mathrm{~nm}$ & $0.18 \mathrm{~nm}$ \\
Instabilitäten $\delta$ & $12.0 \mathrm{~nm}$ & $2.0 \mathrm{~nm}$ \\
Strahldivergenz $\theta_{1 / 2}$ & $1.5 \mathrm{mrad}$ & $0.2 \mathrm{mrad}$ \\
max. Kegelwinkel $\theta_{\max }$ & $150 \mathrm{mrad}$ & $60 \mathrm{mrad}(120 \mathrm{mrad})^{1}$ \\
\hline
\end{tabular}

mit beiden Mikroskopen durchgeführt. Die hochaufösenden Gitterabbildungen (HREM) wurden am Philips CM 200 Elektronenmikroskop aufgenommen. Dabei erfolgte die Abbildung mit einer [110] Zonenachse im symmetrischen 7-Strahlfall. Das Rauschen aus den HREM-Aufnahmen wurde mit einem Fourierfilter zur Verbesserung der Darstellungsqualität der in dieser Arbeit dargestellten Aufnahmen entfernt. Ein Vergleich mit den ungefilterten Aufnahmen stellt sicher, daß die wesentlichen Elemente der dargestellte Struktur durch die Filterung nicht verändert wurden. Die analytische Z-Kontrastabbildung wurde am Philips EM 420 ST durchgeführt. Die Daten der beiden Mikroskope sind in Tabelle 3.3 zusammengefaßt.

Für die analytische elektronenmikroskopische Probencharakterisierung wird in dieser Arbeit die analytische Z-Kontrastabbildung verwendet. Diese Abbildung wird häufig in Rasterelektronenmikroskopen mittels großflächiger Ringdetektoren verwirklicht. Hier wurde jedoch die Realisierung in einem Transmissionselektronenmikroskop über weitwinkelgestreute Elektronen mittels Hollow Cone Beleuchtung eingesetzt. Dabei wird der einfallende Elektronenstrahl entlang eines

\footnotetext{
${ }^{1}$ Spätere Versionen der Steuerungssoftware des Mikroskops lassen einen Kegelwinkel von
} 120 mrad zu, jedoch wurde dies nicht weiter überprüft. 


\section{Experimentelle Methoden}

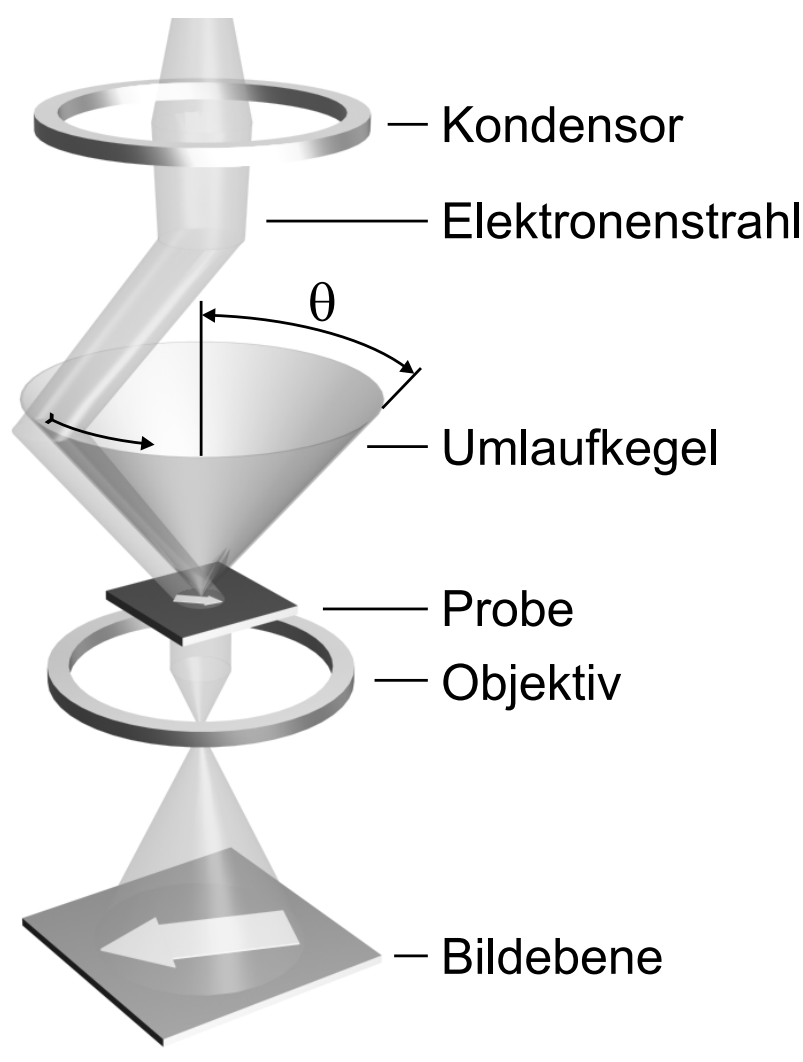

\begin{abstract}
Abb. 3.2. Schematische Darstellung des Strahlengang der Hollow Cone Dunkelfeldabbildung (HCDF) im konventionellen Transmissionselektronenmikroskop. Die Rotation des Strahls entlang des Kegels wurde durch die Ansteuerung mit der im Mikroskop vorhandenen Strahlablenkeinrichtung erreicht.
\end{abstract}

Kegelmantels mit dem Öffnungswinkel $2 \theta$ um die optische Achse des Instruments bewegt. Mit einer Objektivblende, die auf die optische Achse zentriert ist, werden nur Streuanteile aus dem Raumwinkel $\theta$ zur Abbildung verwendet. Die Integration über die verschiedenen Raumwinkel wird bei der Belichtung der Fotoplatte durchgeführt, wobei die Belichtungszeit deutlich größer als die Umlaufzeit des Elektronenstrahls gewählt werden muß. Der geschilderte Strahlengang ist zur Verdeutlichung in Abbildung 3.2 dargestellt.

Die technische Umsetzung erfolgt am EM 420 ST durch eine externe Computersteuerung. Das CM 200 besitzt von vornherein die Möglichkeit zur Hollow Cone Dunkelfeldabbildung. Um die Belichtungszeiten auf unter 20 Sekunden zu halten, wurden große Kondensorblenden $(\emptyset=14 \mathrm{mrad})$ und Objektivblenden $(\emptyset=12 \mathrm{mrad})$ verwendet. Um die für die Auswertung benötigte Elektronendichte $\mathrm{I}_{0}$ einfacher bestimmen zu können, wurde das EM 420 ST um die Möglichkeit erweitert, den abfließenden Strom des Leuchtschirms zu messen. Bei gleichmäBig ausgeleuchtetem Schirm kann die Nullstrahlintensität $I_{0}$ dann leicht ermittelt werden. Da keine CCD Kamera mit entsprechend hoher Pixelanzahl oder ein Bildplattensystem zur Verfügung stand, wurde normales Fotomaterial verwendet. Die Bilder wurden mit einem CCD Scanner digitalisiert und um die logarithmische Übertragungsfunktion des Films korrigiert. 


\section{Quantitative Auswertung der chemischen Analysetechniken}

\subsection{Analytische Z-Kontrastabbildung}

Zur Untersuchung der ausgewählten Fragestellungen ist es wichtig, hochaufösende Methoden zur lokalen Konzentrations- und Strukturbestimmung zu kombinieren. Bei der in dieser Arbeit relevanten Größenskala bietet sich dazu das Transmissionselektronenmikroskop an. Hier können Konzentrations- und Strukturbestimmung direkt kombiniert werden. Jedoch ist eine chemische Analyse mit der geforderten hohen Auflösung unter Berücksichtigung der großen Zahl von Defekten und Verzerrungen in der Analysezone eine sehr anspruchsvolle Aufgabe.

Die klassischen Verfahren der analytischen Elektronenmikroskopie, die Energieverlustspektroskopie (EELS) und die energiedispersive Röntgenspektroskopie (EDS), sind beide mit einem hohen apparativen Aufwand verbunden sind. Es sind spezielle Detektorsysteme und meist auch Feldemissionskathoden erforderlich, um die hohen Stromdichten in den Elektronensonden zu erreichen. Werden die spektroskopischen Techniken im Rasterverfahren eingesetzt, so limitiert der Strahldurchmesser die laterale Auflösung des Verfahrens. Dieser liegt bei Berücksichtigung der für die analytische Messung erforderlichen Strahlintensität bei etwa $1 \mathrm{~nm}$. Mit Energiefiltern ist zudem auch eine energiegefilterte und damit chemisch selektive Abbildung möglich.

Daneben hat sich eine neue Klasse von Techniken etabliert, die aufgrund der Bildintensität unter speziellen Abbildungsbedingungen direkt auf die lokale chemische Zusammensetzung schließen läßt. Die quantitative Bestimmung der lokalen Konzentration ist prinzipiell auf der Basis von hochaufösenden Strukturabbildungen möglich $[77,96,103]$. Jedoch stellen diese Methoden hohe Anforderungen an die Probenqualität und beschränken sich auf die Auswertung von defekt- und verzerrungsfreien Gebieten. Bei den untersuchten Proben sind diese Bedingungen jedoch nicht erfüllt. Daher wurde in dieser Arbeit die Z-Kontrastabbildung verwendet. Sie erfordert keine Strukturabbildung und ist damit auf eine größere Klasse von Fragestellungen anwendbar. Bei der hier vorgestellten Realisierung beträgt das laterale Aufösungsvermögen etwa $1 \mathrm{~nm}$.

Die Hollow Cone Dunkelfeldabbildung (HCDF) wurde seit ihrer ersten Realisierung von Crewe et al. [16] als Ringdetektor im Rasterelektronenmikroskop als aussichtsreicher Kandidat für die Z-Kontrastabbildung gehandelt. Mit aktuellen STEM Geräten wird heutzutage atomare Auflösung erreicht [81]. Jedoch ist der inkohärente Charakter und der Kontrastmechanismus der Z-Kontrastabbildung 


\section{Quantitative Auswertung der chemischen Analysetechniken}

immer noch Diskussionsgegenstand, zum Beispiel bleiben Versetzungen in der HCDF Abbildung sichtbar [82,117]. Die HCDF Abbildung ist somit unter bestimmten Bedingungen keine vollständig inkohärente Abbildung.

Im Rahmen dieser Arbeit wurde deshalb ein Kontrastmodell auf der Basis der Mehrfachstreuung von Elektronen entwickelt, mit der eine quantitative Beschreibung der Bildintensitäten möglich wurde. Dabei wurde besonderer Wert auf die genaue Bestimmung der notwendigen Abbildungsbedingungen gelegt. Im folgenden werden zunächst grundlegende Eigenschaften der Z-Kontrastabbildung untersucht. Aufgrund dieser Erkenntnisse wird dann eine Beschreibung der Bildintensitäten für Systeme aus mehreren Elementen entwickelt und die Genauigkeit der chemischen Analyse überprüft.

\subsubsection{Bildintensitäten der reinen Elemente}

In der Abbildung 4.1 sind vier mit der HCDF Beleuchtung unter verschiedenen Kegelwinkeln gewonnene Z-Kontrastabbildungen eines Kupfer/Gold Schichtpakets auf einem Kupfereinkristall zu sehen. Es ist erkennbar, wie mit steigendem Kegelradius, der links unten in jedem Teilbild angegeben ist, der Spannungsund Orientierungskontrast reduziert wird. Auch nähern sich die Bildintensitäten der Kupferschicht und des Kupfereinkristalls einander an. Mit zunehmender Probendicke, die in jedem Teilbild von rechts nach links zunimmt, erreicht die Bildintensität ein Maximum und wird mit weiter zunehmender Probendicke wieder kleiner. Dieses Maximum verschiebt sich mit größeren Kegelwinkeln zu größeren

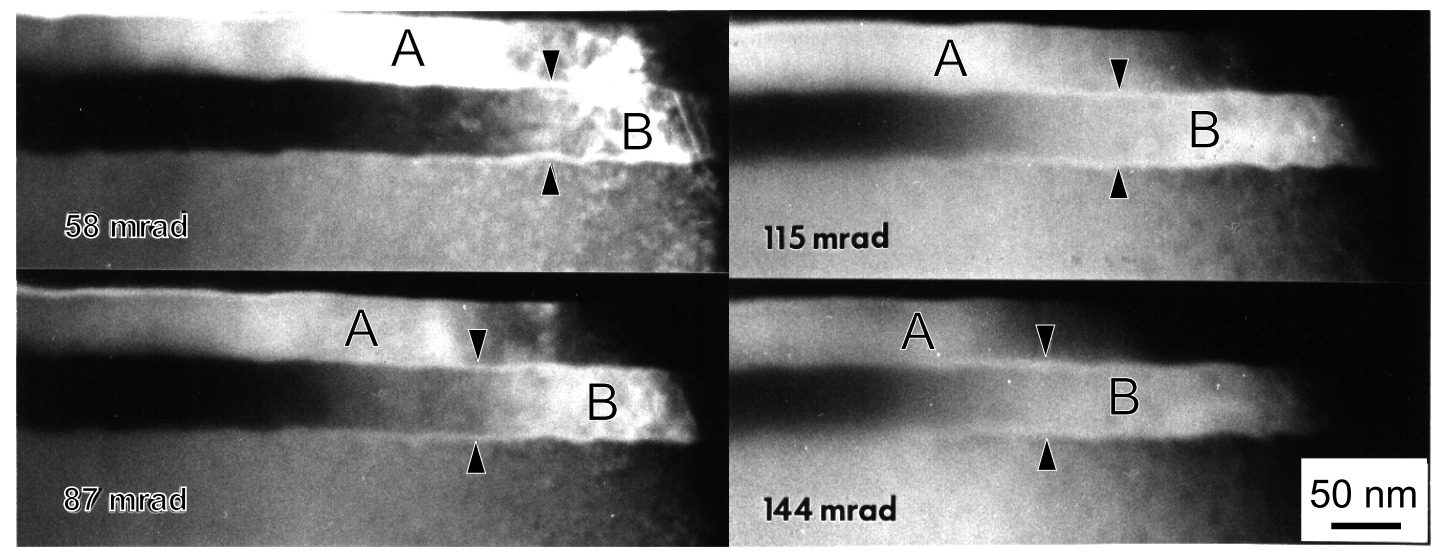

Abb. 4.1. Z-Kontrastabbildung eines $\mathrm{Cu} / \mathrm{Au} / \mathrm{Cu}$ Schichtsystems im Querschnitt. Die vier Bilder stellen dieselben Probenstellen bei unterschiedlichem Kegelradius, der links unten in jedem Teilbild angegeben ist, dar. Die Probendicke nimmt von rechts nach links zu. An der Grenzfläche ist, durch die Pfeile gekennzeichnet, eine Überhöhung der Intensität zu beobachten. 


\section{Quantitative Auswertung der chemischen Analysetechniken}

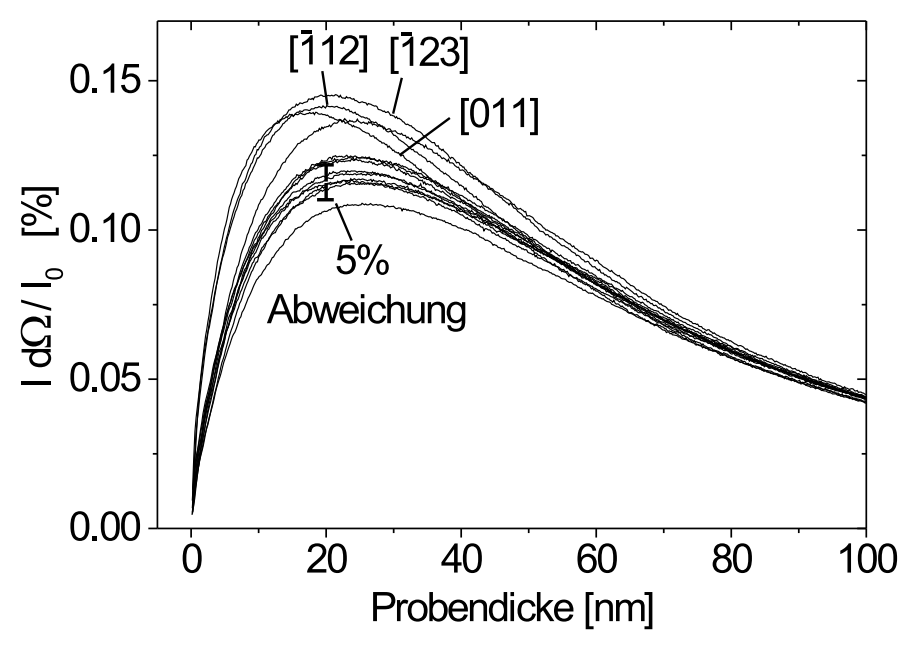

Abb. 4.2. Intensität der HCDF Abbildung für verschiedene Probenorientierungen einer einkristallinen Goldprobe bei einem Kegelwinkel von $144 \mathrm{mrad}$. Die Probe wurde von einer [011] $\mathrm{zu}$ einer [1112] Zonenachse um eine [111] Drehachse verkippt. Eine genauere Diskussion ist in [92] zu finden.

Probendicken. Damit ergibt sich ein kleiner Kontrast zwischen dem Maximum der Bildintensität in Kupfer (A) und in Gold (B) und eine Kontrastinversion zu noch größeren Probendicken. Die durch Pfeile gekennzeichneten Probenstellen weisen eine erhöhte Bildintensität an der Grenzfläche auf. Diese Eigenschaft der Z-Kontrastabbildung wird bei $[22,38,92]$ eingehend diskutiert. Für eine chemische Analyse sollten aufgrund des reduzierten Spannungs- und Orientierungskontrasts möglichst große Kegelwinkel verwendet werden.

Um den Einfluß verschiedener Probenorientierungen auf die Bildintensitäten in der Z-Kontrastabbildung zu untersuchen, wurde eine einkristalline Eichprobe aus Gold hergestellt. Diese Probe wurde in 15 Schritten von [011] nach [112] gekippt und die Bildintensität vermessen. Die Veränderung der Probendicke aufgrund der Kippung wurde korrigiert. Die Probendicke wurde aus Extinktionskonturen im Vergleich mit Blochwellenrechnungen an niedrigindizierten, experimentell gut einstellbaren Zonenachsen ermittelt. Bei der Auswahl der Probenstelle für die Eichmessung wurde auf eine definierte Keilform geachtet, um die Probendicke über den Bereich der Extinktionskonturen extrapolieren zu können. Wie in Abbildung $4.2 \mathrm{zu}$ sehen ist, in der die 15 Kurven der einzelnen Orientierungen dargestellt sind, ergibt sich eine außerordentliche Erhöhung der Bildintensität für einige Orientierungen. Für die anderen Probenorientierungen liegt die Variation unterhalb von 5\%. Wie Abbildung 4.3 zeigt, findet man diese Überhöhungen der Bildintensität speziell bei Orientierungen, die mit niedrig indizierten Lauezonen verknüpft sind. Bei einer quantitativen Auswertung sollten daher diese speziellen Orientierungen vermieden und kinematische Anregungsbedingungen gewählt werden, da eine leichte Variation der Orientierung sonst zu einer starken Änderung der Bildintensität und damit zu einer Beeinträchtigung der chemischen Analyse führt. 
4. Quantitative Auswertung der chemischen Analysetechniken

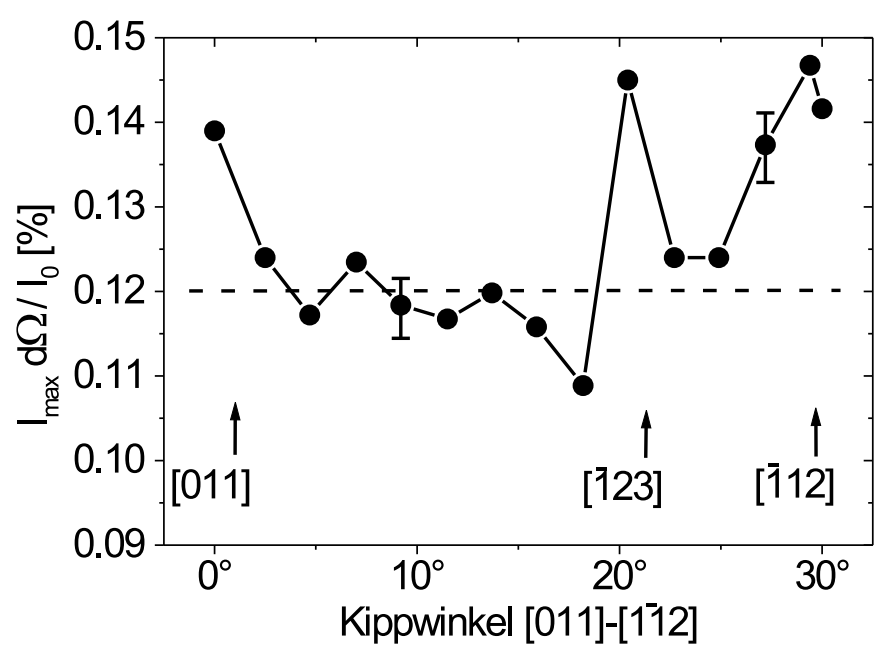

Abb. 4.3. Die Bildintensität der Maxima der HCDF Abbildung in Abhängigkeit von der Probenorientierung. Die Bildintensität ist bei den niedrig indizierten Zonenachsen deutlich erhöht.

\subsubsection{Inkohärenz der HCDF Abbildung}

Wie gezeigt, kann die HCDF Abbildung bei Verwendung großer Kegelwinkel und ausgewählter Probenorientierungen dynamische Kontraste wirksam unterdrücken. Für die Beschreibung im Rahmen eines Kontrastmodells ist es nun von entscheidender Bedeutung, ob es sich bei der HCDF Abbildung tatsächlich um eine inkohärente Abbildung handelt. Ist dies der Fall, kann die Bildintensität additiv aus den von den einzelnen Streuzentren gestreuten Intensitäten zusammengesetzt werden, was eine Modellierung erheblich vereinfacht. Treacy und Gibson [111,112] und Hartel et al. [37] haben den Bildentstehungsprozeß beim Z-Kontrast mit der Hollow Cone Beleuchtung und einem Ringdetektor im STEM untersucht. Diese Arbeiten zeigen, daß die Inkohärenz der Abbildung von der der Beleuchtung bzw. der Detektorgeometrie wie auch durch inkohärente Streuprozesse verursacht werden kann. Je nach Abbildungsbedingungen kann der eine oder andere Prozeß dominieren. Folgt man Treacy und Gibson [111,112], so erzeugt eine Probe die folgende Bildintensität

$$
I(\theta)=2 \pi|f(\theta)|^{2}\left\{\sum_{j}\left|a_{j}\right|^{2}+\sum_{j} \sum_{l>j} a_{j} a_{l}^{*} V_{c} \cdot e^{-2 M}\right\},
$$

wobei $f(\theta) \cdot a_{i}$ für die Bildamplitude des Atoms i steht und die Summe über alle Atome, die zur Bildentstehung beitragen, zu bilden ist. Die thermischen Schwingungen der Atome werden durch den Debye-Waller Faktor $e^{-2 M}, M=$ $\left(8 \pi^{2} / \lambda^{2}\right) \sin ^{2}(\theta / 2)<u_{x}^{2}>$ berücksichtigt, wobei $<u_{x}^{2}>$ das mittlere Verschiebungsquadrat bezeichnet. Damit kann ein Kohärenzvolumen $V_{c}$ wie folgt definiert werden

$$
V_{c}\left(x_{j l}, z_{j l}, \theta\right)=\int_{\theta_{1}}^{\theta_{2}} J_{0}\left(2 \pi x_{j l} \sin (\theta) / \lambda\right) \cdot \cos \left(4 \pi z_{j l} \sin ^{2}(\theta / 2) / \lambda\right) d \theta
$$


4. Quantitative Auswertung der chemischen Analysetechniken
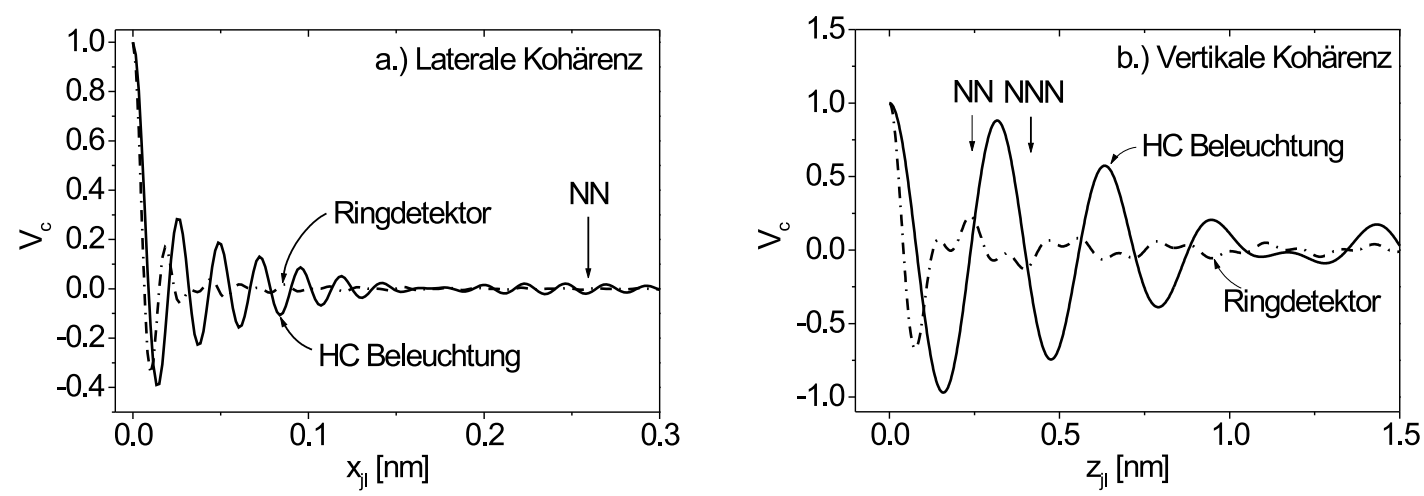

Abb. 4.4. Kohärenzvolumen $V_{c}$ aus Gleichung 4.2 berechnet für (a) eine laterale und (b) eine vertikalen Richtung parallel zur optischen Achse. Die durchgezogene Linie entspricht der Hollow Cone Beleuchtung $\left(\theta_{1}=135 \mathrm{mrad}, \theta_{2}=155 \mathrm{mrad}\right)$. Zum Vergleich wird ein typischer Ringdetektor eines STEM $\left(\theta_{1}=150 \mathrm{mrad}, \theta_{2}=250 \mathrm{mrad}\right) \mathrm{mit}$ der gestrichelten Linie angegeben. Der nächste $(\mathrm{NN})$ und übernächste Nachbarabstand (NNN) in Kupfer ist durch Pfeile markiert.

wobei $x_{j l}$ und $z_{j l}$ der laterale und vertikale Abstand zwischen zwei streuenden Atomen $\mathrm{j}$ und $\mathrm{l}$, und $J_{0}$ die Besselfunktion nullter Ordnung bezeichnen. Die Bildamplituden zweier Atome $\mathrm{j}$ und $\mathrm{l}$ interferieren nur dann, wenn für ihren Abstand $x_{j l}$ und $z_{j l}$ das Kohärenzvolumen $V_{c}\left(x_{j l}\right)$ oder $V_{c}\left(z_{j l}\right)$ signifikant von Null abweicht. In Abbildung 4.4 ist das sich aus Gleichung 4.2 ergebende Kohärenzvolumen $V_{c}$ entlang einer lateralen Richtung (a) und der optischen Achse (b) aufgetragen. Die durchgezogene Linie entspricht jeweils den Bedingungen, wie sie in dieser Arbeit bei der HCDF Abbildung verwendet wurden, die gestrichelte Linie entspricht jeweils einem typischen Ringdetektor im STEM. Wie man sieht, ist die laterale Kohärenz für beide Abbildungsbedingungen am Ort des nächsten Nachbarn zerstört und diese können nicht mehr kohärent wechselwirken. Damit sind von der lateralen Kohärenz keine Oszillationen der Intensitäts-Dicken Beziehung zu erwarten. Dagegen ist die vertikale Kohärenz an den Stellen des nächsten und übernächsten Nachbarn für die HCDF Abbildung nahezu vollständig erhalten. Damit ergeben sich aus der Geometrie der HCDF Abbildung keine inkohärenten Abbildungsbedingungen.

Im Falle einer Zonenachse sollte die Gleichung 4.2 konstruktive und destruktive Interferenz mit zunehmendem Streuwinkel zur Folge haben. Die experimentell ermittelten Intensitätsdaten bei steigendem Kegelwinkel sind dazu in Abbildung 4.5 aufgetragen. Die Kegelwinkel, bei denen sich nach Gleichung 4.2 konstruktive und destruktive Interferenzen ergeben sollten, sind in der Grafik durch Pfeile markiert. Da keine Oszillationen bei den theoretisch erwarteten Winkeln auftreten, wird offensichtlich auch die vertikale Kohärenz in der HCDF Abbildung wirksam unterdrückt. 
4. Quantitative Auswertung der chemischen Analysetechniken

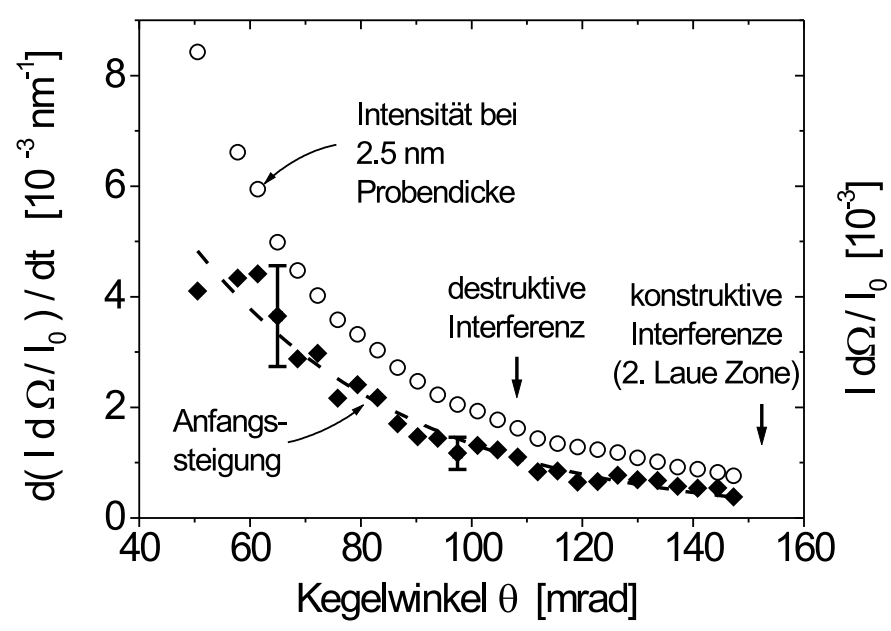

Abb. 4.5. Steigung der linearen Intensitäts-Dicken Beziehung in dünnem Gold mit zunehmendem Kegelwinkel. Zusätzlich ist die Intensität für eine konstante Probendicke von $2.5 \mathrm{~nm}$ angegeben. Die Pfeile markieren die Kegelwinkel, bei denen konstruktive und destruktive Interferenz auftreten könnte.

Tab. 4.1. Debye-Waller Faktoren für verschiedene Metalle, ermittelt aus der mittleren Bewegungsamplitude bei Raumtemperatur aus [87].

\begin{tabular}{|cc|c|c|c|c|}
\hline & & $\mathrm{Al}$ & $\mathrm{Cu}$ & $\mathrm{Ag}$ & $\mathrm{Au}$ \\
\hline \hline$\left.<u_{x}^{2}\right\rangle$ & {$\left[10^{-4} \mathrm{~nm}^{2}\right]$} & 1.05 & 0.69 & 0.84 & 0.74 \\
\hline$e^{-2 M}$ & {$\left[10^{-3}\right]$} & 0.48 & 6.5 & 2.2 & 4.6 \\
\hline
\end{tabular}

Die Kohärenz muß somit durch den Streuvorgang an sich zerstört werden. Dies wird durch inelastische Prozesse wie Anregung von Plasmonen und Elektronen auf inneren Schalen oder Comptonstreuung bzw. quasi-elastische thermisch diffuse Streuung erreicht. Die Debye-Waller Faktoren aus Tabelle 4.1, mit denen man den Grad der Kohärenz abschätzen kann, ergeben für verschiedene Metalle bei einem Kegelwinkel von $144 \mathrm{mrad}$ Werte unter 1\%. Damit wird der kohärente Anteil $e^{-2 M}$ der Bildintensität aus Gleichung 4.1 soweit reduziert, daß er keinen wesentlichen Beitrag mehr leisten kann.

Umgekehrt sind die Debye-Waller Faktoren aber so klein, daß sie die verbleibende Abhängigkeit der Intensitäten von der Probenorientierung bei niedrig indizierten Probenorientierungen nicht erklären können. Die Debye-Waller Faktoren aus Tabelle 4.1 scheinen die Unterdrückung der Kohärenz deutlich zu unterschätzen. Hier scheint die Mehrfachstreuung schon bei geringen Probendicken eine Rolle zu spielen. Spaltet man ein Streuereignis in zwei Streuereignisse kleineren Winkels auf, so erhält man für den Debye-Waller Faktor einen größeren Wert:

$$
e^{-2 M\left(\theta_{1}\right)} \times e^{-2 M\left(\theta_{2}\right)} \geq e^{-2 M\left(\theta_{1}+\theta_{2}\right)}
$$

Die Mehrfachstreuung sorgt demzufolge dafür, daß die kohärenten Beugungseffekte nicht mehr so gut unterdrückt werden können. Es zeigt sich, daß es sich 


\section{Quantitative Auswertung der chemischen Analysetechniken}

bei der HCDF Abbildung weder um eine kohärente noch eine vollständig inkohärente Abbildung handelt. Für eine möglichst ideale Z-Kontrastabbildung sollte bei der HCDF Beleuchtung eine Probenorientierung gewählt werden, bei der im komplementären Hellfeldbild kinematische Anregungsbedingungen vorliegen.

\subsubsection{Quantitative Beschreibung der Bildintensitäten}

Die HCDF Abbildung ist unter den in vorangegangenen Kapitel angegebenen Bedingungen in der Lage, dynamische Kontraste zu unterdrücken. Die gefundene Beziehung zwischen der Bildintensität und der Probendicke ist jedoch nicht linear, wie dies für eine ideale Z-Kontrastabbildung zu erwarten wäre. Die hier angewendete Beschreibung berücksichtigt keine Blochwellen. Mehrfachstreuung wie von Treacy et al. $[111,112]$ angenommen wird jedoch berücksichtigt.

Im folgenden wird die Bildentstehung in einem inkohärenten Mehrfachstreumodell formuliert. Das im Rahmen dieser Arbeit entwickelte Modell baut auf den Arbeiten von Goudsmit und Saunderson auf [33]. Mit dem differentiellen Streuquerschnitt $\mathrm{d} \sigma / \mathrm{d} \Omega$, dem totalen Streuquerschnitt $\sigma_{\text {tot }}$, dem normierten Streuquerschnitt $S_{1}(\theta)=1 / \sigma_{\text {tot }} \cdot d \sigma / d \Omega$, der mittleren freien Weglänge $\Lambda$ zwischen zwei Streuereignissen, $P_{n}$ einem Legendre Polynom der n-ten Ordnung und den Koeffizienten

$$
a_{n}=2 \pi \int_{0}^{\pi} S_{1}(\theta) P_{n}(\cos \theta) \sin \theta d \theta
$$

ergibt sich die Winkelverteilung im Raumsegment $d \Omega$ nach einer m-fachen Streuung zu

$$
S_{m}(\theta) d \Omega=\frac{d \Omega}{4 \pi} \sum_{n=0}^{\infty}(2 n+1) a_{n}^{m} \cdot P_{n}(\cos \theta)
$$

Die Wahrscheinlichkeit einer m-fach Streuung entlang eines Elektronenpfades mit der Länge t entspricht einer Poissonverteilung. Damit ergibt sich eine Bildintensität der mehrfachgestreuten Elektronen unter dem Kegelwinkel $\theta$ mit $\mathrm{p}=\mathrm{t} / \Lambda$ $\mathrm{zu}$

$$
\frac{I(t, \theta) d \Omega}{I_{0}}=\frac{d \Omega}{4 \pi} \sum_{n=0}^{\infty}(2 n+1) P_{n}(\cos \theta) \cdot e^{-p}\left(e^{p \cdot a_{n}}-1\right)
$$




\section{Quantitative Auswertung der chemischen Analysetechniken}

Die Anzahl der Koeffizienten $a_{n}$, die man für eine gute Näherung benötigt, ist aufgrund der langsamen Konvergenz der Summe mit 500-1000 recht hoch. Da dieses Modell weder Energieverlust noch Absorption berücksichtigt, ergibt sich für eine unendlich dicke Probe eine Gleichverteilung der Intensität in alle Raumrichtungen:

$$
\lim _{t \rightarrow \infty} \frac{I(t, \theta) d \Omega}{I_{0}}=\frac{d \Omega}{4 \pi}
$$

Im Grenzfall einer unendlich dünnen Probe kann der differentielle Streuquerschnitt für die jeweilige Abbildungsbedingung aus der Anfangssteigung der gemessenen Intensitäts-Probendicken Beziehung wie folgt ermittelt werden:

$$
\lim _{t \rightarrow 0} \frac{d}{d t} \frac{I(t, \theta) d \Omega}{I_{0}}=\frac{d \Omega}{4 \pi} \sum_{n=0}^{\infty}(2 n+1) P_{n}(\cos \theta) \cdot \frac{a_{n}}{\Lambda}=N_{v} \cdot \frac{d \sigma}{d \Omega} d \Omega
$$

Dabei bezeichnet $N_{v}$ die Streuzentrendichte. Für eine quantitative Auswertung müssen somit die Geometrie der experimentellen Abbildung und die Nullstrahlintensität $I_{0}$ genau bekannt sein.

Mit diesem Mehrfachstreumodell lassen sich die experimentell gemessenen Intensitätsverläufe und die Verschiebung der Intensitätsmaxima zu größeren Probendicken bei größeren Kegelwinkeln beschreiben. Als Parameter verwendet das Mehrfachstreumodell für ein Element die mittlere freie Weglänge $\lambda$ und den normierten Streuquerschnitt $S_{1}(\theta)$. Die Güte dieser Parameter beeinflußt die Genauigkeit, mit der die Bildintensitäten reproduziert werden können, deutlich. Aus der Literatur wurden die Streuquerschnitte von Doyle-Turner [19] und Wenzel [119] für das Element Gold entnommen. Aus diesen Daten wurden die Bildintensitäten, die sich aus den Streuquerschnitten im Rahmen des Mehrfachstreumodells ergeben, berechnet und in Abbildung 4.6 zusammen mit den experimentellen Daten aufgetragen. Es ergibt sich eine eher schlechte Übereinstimmung mit den experimentellen Daten. Beide Modelle ergeben ein Intensitätsmaximum bei 2-5 mal größeren Probendicken. Diese Abweichung ist zu groß, um sie mit Abweichungen bei der Eichung der Probendicken, die über Extinktionskonturen ermittelt wurden, erklären zu können. Da die Probendicke im Mehrfachstreumodell über den Faktor $p=t \cdot N_{v} \cdot \sigma_{t o t}$ eingeführt wird, kann man die Probendicke $t$ über den totalen Streuquerschnitt $\sigma_{\text {tot }}$ und damit der freien Weglänge $\Lambda$ anpassen. Entsprechende Werte sind in Tabelle 4.2 angegeben. Da die notwendigen freien Weglängen viel kleiner sind, als in der Literatur für Gold angegeben, muß der Verlauf des Streuquerschnitts in Frage gestellt werden. Dazu werden die Streuquerschnitte mit in dieser Arbeit bestimmten experimentellen Daten verglichen. Der normierte differentielle Streuquerschnitt wurde aus dünnen Probenstellen nach dem Zusammenhang aus Gleichung 4.8 für verschiedene Streuwinkel ermittelt und in 


\section{Quantitative Auswertung der chemischen Analysetechniken}

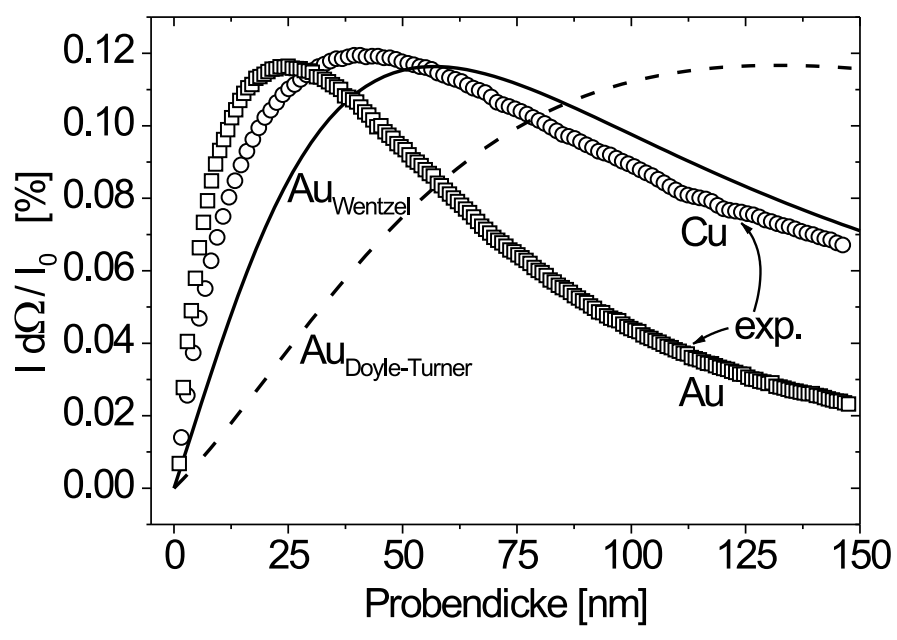

Abb. 4.6. Experimentelle Intensitätsdaten von reinem Gold und Kupfer als Funktion der Foliendicke. Die gestrichelte und die durchgezogene Linie wurden aus dem Doyle-Turner [19] und dem Wentzel [119] Streuquerschnitt im Rahmen des Mehrfachstreumodells für Gold errechnet. Die Modellparameter finden sich in Tabelle 4.2 .

Tab. 4.2. Im Rahmen des Mehrfachstreumodells verwendeten freien Weglängen und Parameter der Streuquerschnitte von Doyle-Turner [19], Wentzel [119] und dieser Arbeit. Die Parameter b und $\gamma$ des angepaßten Streuquerschnitts beziehen sich auf Gleichung 4.9 .

\begin{tabular}{|c|c|c|c|c|c|c|c|}
\hline \multirow[t]{2}{*}{ Element } & \multirow[t]{2}{*}{$\mathrm{Z}$} & \multirow{2}{*}{$\begin{array}{c}N_{v} \\
{\left[\mathrm{~nm}^{-3}\right]}\end{array}$} & \multicolumn{2}{|c|}{$\begin{array}{c}\text { Doyle-Turner [19] } \\
\text { Wentzel [119] }\end{array}$} & \multicolumn{3}{|c|}{$\begin{array}{c}\text { angepaßter } \\
\text { Streuquerschnitt }\end{array}$} \\
\hline & & & $\begin{array}{c}\Lambda \\
{[\mathrm{nm}]}\end{array}$ & $\begin{array}{c}\Lambda \text { (angepaßt) } \\
{[\mathrm{nm}]}\end{array}$ & $\mathrm{b}$ & $\gamma$ & $\begin{array}{c}\Lambda \\
{[\mathrm{nm}]}\end{array}$ \\
\hline $\mathrm{Cu}$ & 29 & 85 & $\begin{array}{l}34 \\
25\end{array}$ & $\begin{array}{l}2.8 \\
4.4\end{array}$ & 58.0 & 0.868 & 6.0 \\
\hline $\mathrm{Au}$ & 79 & 59 & $\begin{array}{l}11 \\
9.2\end{array}$ & $\begin{array}{l}1.9 \\
3.7\end{array}$ & 25.0 & 0.912 & 4.75 \\
\hline
\end{tabular}

Abbildung 4.7 aufgetragen. Dabei erkennt man, daß die Streuquerschnitte von Doyle-Turner und Wentzel die Streuung bei großen Winkeln unterschätzen. Im Fall von Doyle-Turner, die den Streuquerschnitt mit mehreren Gaussfunktionen beschreiben und deren Streuquerschnitt im Grenzfall großer Winkel nicht dem Verhalten eines Rutherfordstreuquerschnitts entspricht, ist dies zu erwarten. Im Fall des Streuquerschnitts von Wentzel, daß ein korrektes Verhalten im Grenzfall großer Winkel aufweist, ist die Abweichung zu den experimentellen Daten zwar kleiner, aber immer noch signifikant.

Es bleibt festzuhalten, daß die Streuquerschnitte, die in der Literatur zu finden sind und im wesentlichen bei kleinen Winkelbereichen der konventionellen Abbildung erprobt sind, die gemessenen Bildintensitäten nicht korrekt beschreiben können. Dies ist jedoch Voraussetzung für die quantitative, chemische Analyse 
4. Quantitative Auswertung der chemischen Analysetechniken

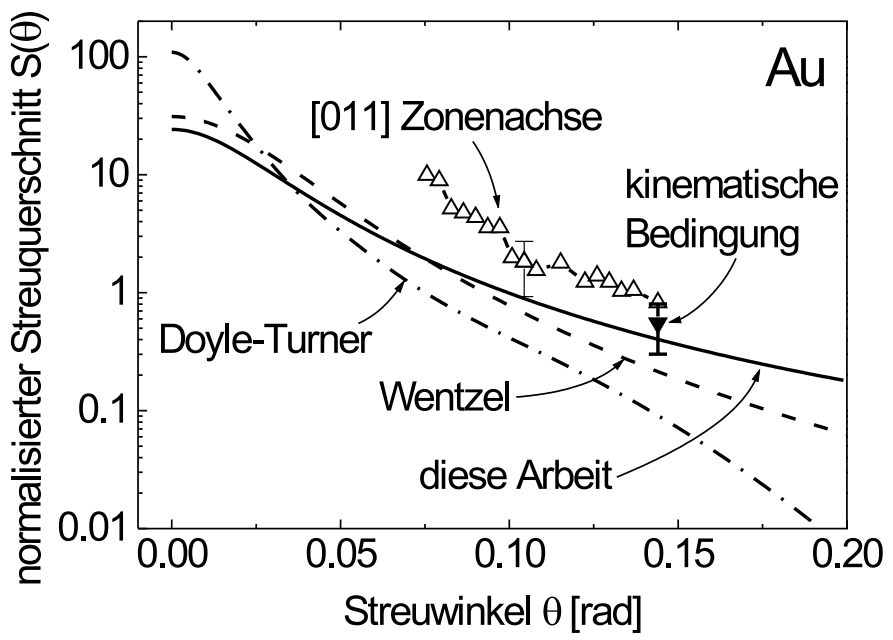

Abb. 4.7. Normierte differentielle Streuquerschnitte für Gold nach Doyle-Turner [19], Wentzel [119] und aus dieser Arbeit. Zum Vergleich sind experimentelle Daten aus Z-Kontrastaufnahmen $\mathrm{zu}$ sehen. Selbst im Fall von kinematischen Abbildungsbedingungen ist die Streuung bei großen Winkeln stärker als von der Literatur vorhergesagt.

von Aufnahmen in der Z-Kontrastabbildung. Deshalb soll im folgenden auf Basis eigener experimenteller Ergebnisse ein passender Streuquerschnitt angepaßt werden.

Prinzipiell könnte eine hinreichende Menge an Koeffizienten $a_{n}$ aus Gleichung 4.5 an die experimentellen Daten angepaßt werden. Wie in einer vorangegangenen Arbeit gezeigt wurde, ist dieses Konzept dazu geeignet, die Abhängigkeit der Intensität von der Probendicke bei unterschiedlichen Kegelwinkeln mit einem Parametersatz zu beschreiben [38,22]. Die Methode ist jedoch numerisch instabil und führt zu künstlich oszillierenden Streuquerschnitten.

In Abbildung 4.8 sind die Koeffizienten von Gold nach Gleichung 4.4 für die Streuquerschnitte von Doyle-Turner [19] und Wentzel [119] aufgetragen. Für beide Streuquerschnitte fallen die Werte der Koeffizienten $a_{n}$ mit zunehmendem n. Der

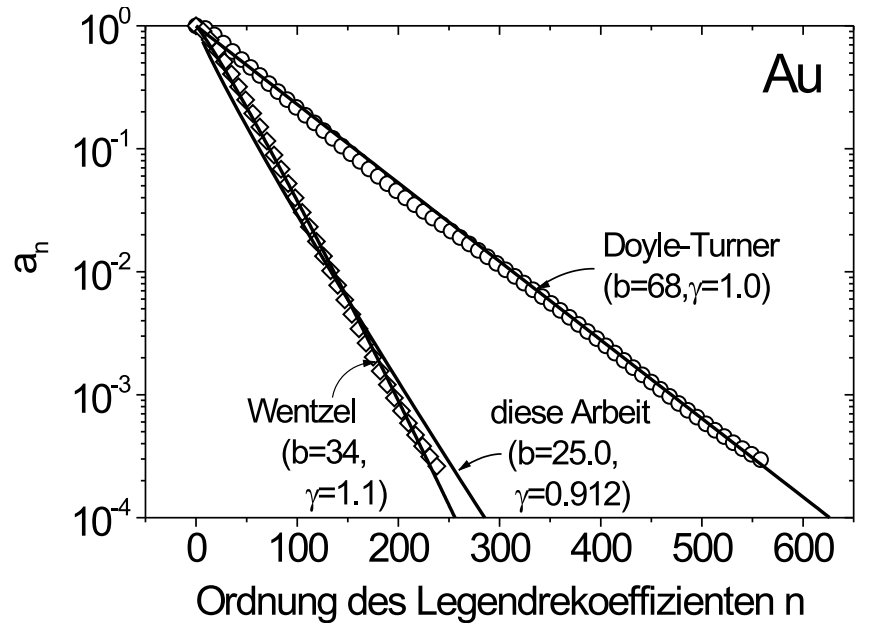

\begin{abstract}
Abb.
4.8. LegendreKoeffizienten $a_{n}$ für die Streuquerschnitte von DoyleTurner [19] und Wentzel [119] für Gold. Die Kreise sind aus den zugehörigen Streuquerschnitten nach Gleichung 4.4 ermittelt worden. Die durchgezogenen Linien entspricht einer Anpassung an die Gleichung 4.9. Zusätzlich wurde der in dieser Arbeit für Gold ermittelte Streuquerschnitt angegeben.
\end{abstract}


4. Quantitative Auswertung der chemischen Analysetechniken

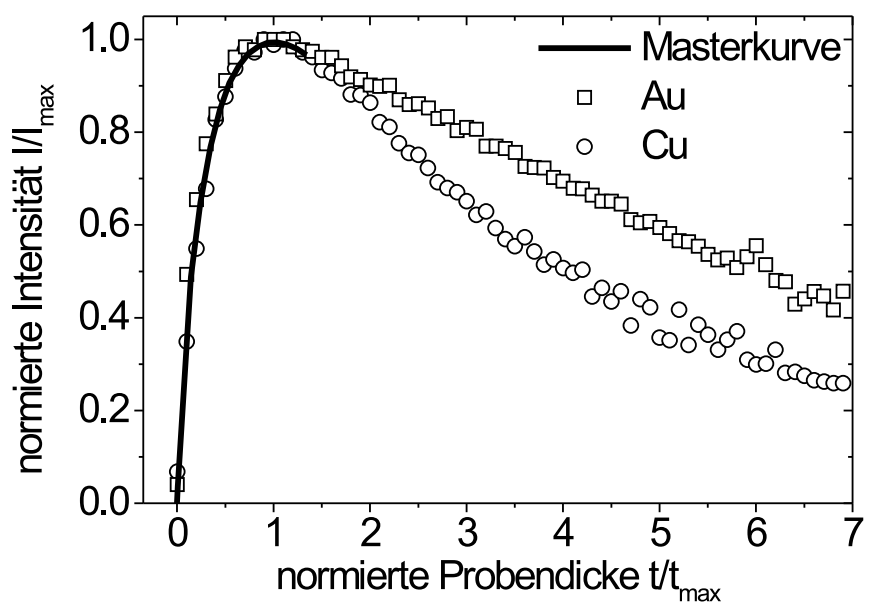

Abb. 4.9. Normierter Intensitätsverlauf von Kupfer und Gold bei Kegelwinkeln von 72 bis 144 mrad über der normierten Probendicke. Die verschiedenen Intensitätsverläufe liegen im Rahmen der experimentellen Genauigkeit vor dem Intensitätsmaximum auf einer universellen Masterkurve.

Koeffizient 0-ter Ordnung hat aufgrund der Normierung von $S_{1}$ den Wert von 1. Die Folge der $a_{n}$ und damit der Streuquerschnitt kann hierbei gut durch folgenden Zusammenhang approximiert werden:

$$
a_{n}=e^{-\left(\frac{n}{b}\right)^{\gamma}}
$$

Mit der Gleichung 4.9 wird die Zahl der Parameter zur Beschreibung des Streuquerschnitts auf zwei Parameter b und $\gamma$ reduziert. Dadurch ergeben sich physikalisch sinnvolle Streuquerschnitte mit einer Winkelabhängigkeit ähnlich zu dem von Wentzel angegebenen. Aufgrund dieser Parametrisierung eines Streuquerschnitts kann nun versucht werden, einen zu den experimentellen Ergebnissen passenden Streuquerschnitt zu finden.

\subsubsection{Anpassung eines Streuquerschnitts}

Zur Anpassung des Streuquerschnitts an die experimentellen Daten muß man zunächst die Merkmale des Intensitäts-Probendicken Zusammenhangs I(t) auswählen, an die ein Streuquerschnitt am besten angepaßt werden kann. Bei der Untersuchung von I(t) fällt auf, daß die Kurven immer eine ähnliche Form aufweisen. Normiert man die Kurven auf das Maximum, so ergibt sich eine Masterkurve wie in Abbildung 4.9 dargestellt. Eine Abweichung von dieser Kurve findet man für Probendicken, die über das Intensitätsmaximum hinausgehen. Die Kurvenform bis zum Intensitätsmaximum ist jedoch nahezu unabhängig vom Probenmaterial und dem bei der Abbildung verwendeten Kegelwinkel. Ein angepaßter Streuquerschnitt muß diesen Verlauf reproduzieren.

In Abbildung 4.10 sind (a) die Probendicke $t_{\max }$ und (b) die Intensität des Intensitätsmaximums $I_{\max }$ gegenüber den verschiedenen Kegelwinkeln aufgetragen. 
4. Quantitative Auswertung der chemischen Analysetechniken
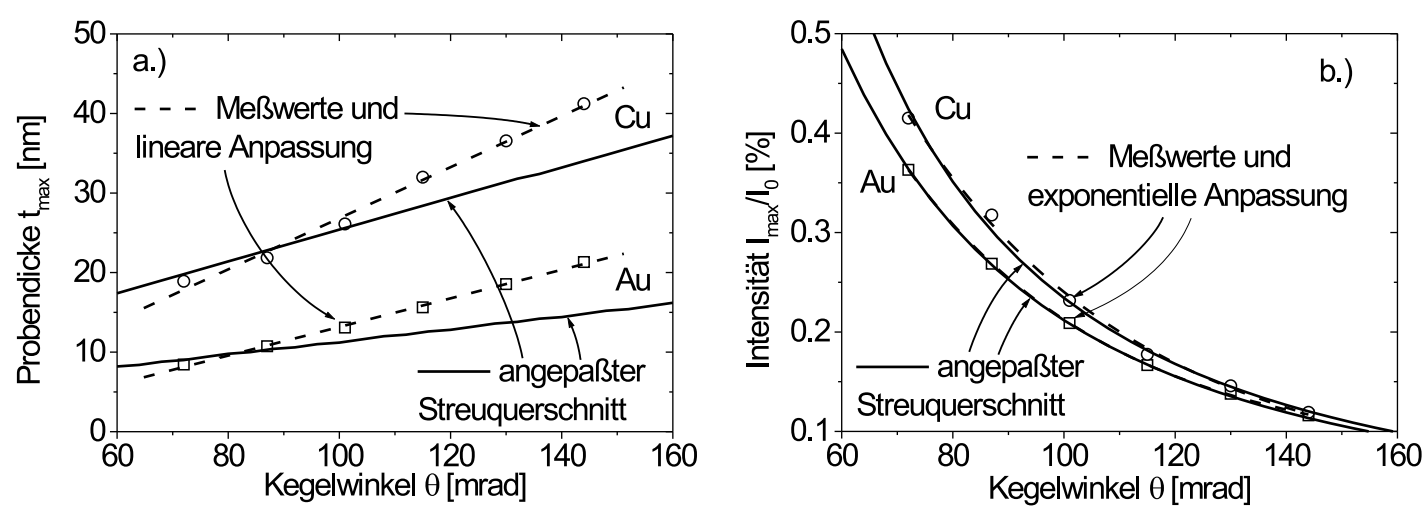

Abb. 4.10. Die Probendicke bei maximaler Intensität (a) und die maximale Intensität (b) bei verschiedenen Kegelwinkeln. Die Position des Intensitätsmaximums variiert linear mit dem Kegelwinkel im Bereich von 72 bis 144 mrad. Die maximale Intensität fällt exponentiell mit zunehmendem Kegelwinkel ab.

Die Probendicke bei maximaler Intensität $t_{\max }$ hängt dabei linear, die Intensität $I_{\text {max }}$ exponentiell vom Kegelwinkel ab. Auch diese Abhängigkeiten müssen durch einen korrekten Streuquerschnitt wiedergeben werden.

Wie die Abbildung 4.9 und 4.10 gezeigt haben, ist die Form des Intensitätsverlaufs für unterschiedliche Elemente sehr ähnlich, jedoch findet man eine stärkere Abhängigkeit beim Verlauf des Intensitätsmaximums bei Veränderung des Kegelwinkels. Deshalb hat es sich bewährt den Streuquerschnitt an den Verlauf des Maximums mit dem Kegelwinkel anzupassen. Dazu wurden für Streuquerschnitte mit den Parametern b von 3 bis 40 und $\gamma$ von 0.3 bis 5 aus dem Mehrfachstreumodell zwei typische Merkmale, die Änderung der Probendicke des Intensitätsmaximums $f 1 \sim d t_{\max } / d \theta$ und der exponentiellen Abbfall der Maximalintensität $I_{\text {max }} \sim e^{-\left(\theta-\theta_{0}\right) / f 2}$ mit steigendem Kegelwinkel $\theta$ bestimmt und in Abbildung 4.11 aufgetragen.

Einen passenden Streuquerschnitt ermittelt man aus beiden Graphen durch Überlagerung der Isokonturlinien der experimentell gefundenen Werte für f1 und f2. Der Schnittpunkt der beiden Kurven liefert die Parameter für den Streuquerschnitt. Im Fall von Gold ergibt sich aus Abbildung 4.12 ein Wert von b = 26 und $\gamma=0.87$.

Da der bestimmte Parametersatz die absoluten Werte für den linearen Anstieg der Probendicke bezüglich der Maximalintensität und des exponentiellen Abfalls der Maximalintensität mit zunehmendem Kegelwinkel $\theta$ nicht gleich gut beschreibt, wurde auf eine optimale Anpassung der maximalen Intensitätswerte geachtet. Diese können experimentell viel genauer bestimmt werden als die Probendicke, die aus den Dickenkonturen im Hellfeldbild unter der Annahme einer Probe mit konstantem Probenkeil in den Bereich großer Dicken extrapoliert werden mußte. 


\section{Quantitative Auswertung der chemischen Analysetechniken}
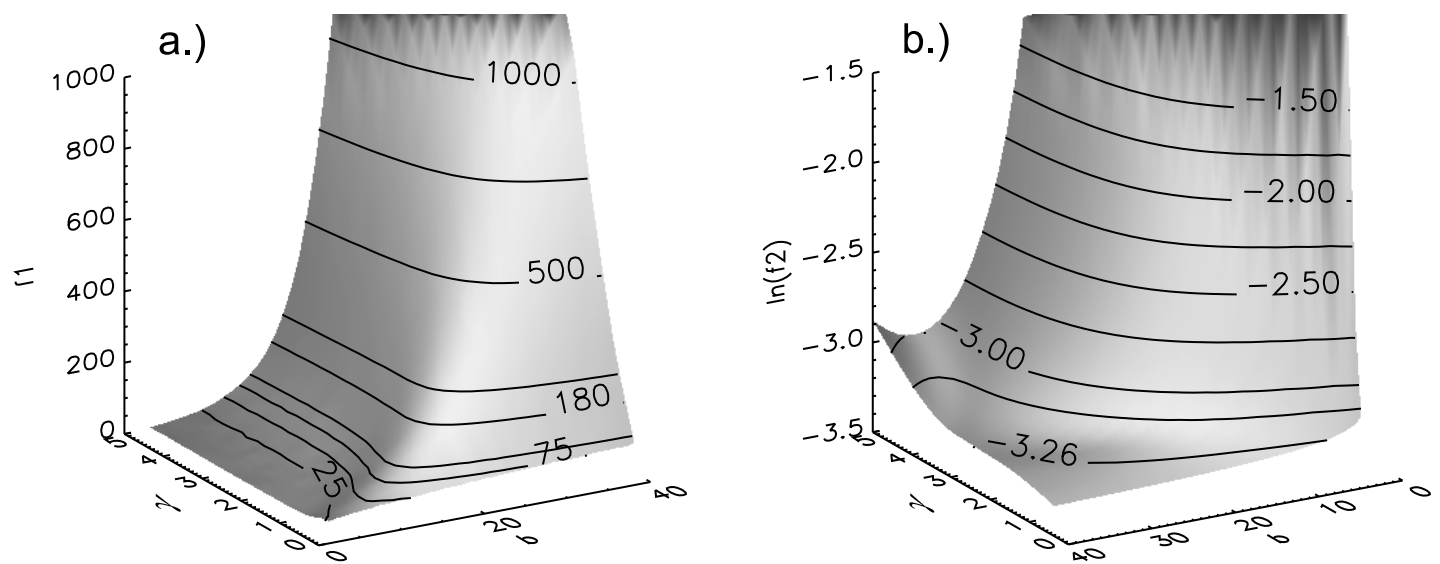

Abb. 4.11. Die Änderung der Probendicke des Intensitätsmaximums $f 1 \sim d t_{\max } / d \gamma$ (a) und der exponentielle Abfall der Maximalintensität $I_{\max } \sim e^{-\left(\theta-\theta_{0}\right) / f 2}$ (b) wurden für das Parameterfeld $\mathrm{b}$ von 3 bis 40 und $\gamma$ von 0.3 bis 5 bestimmt.

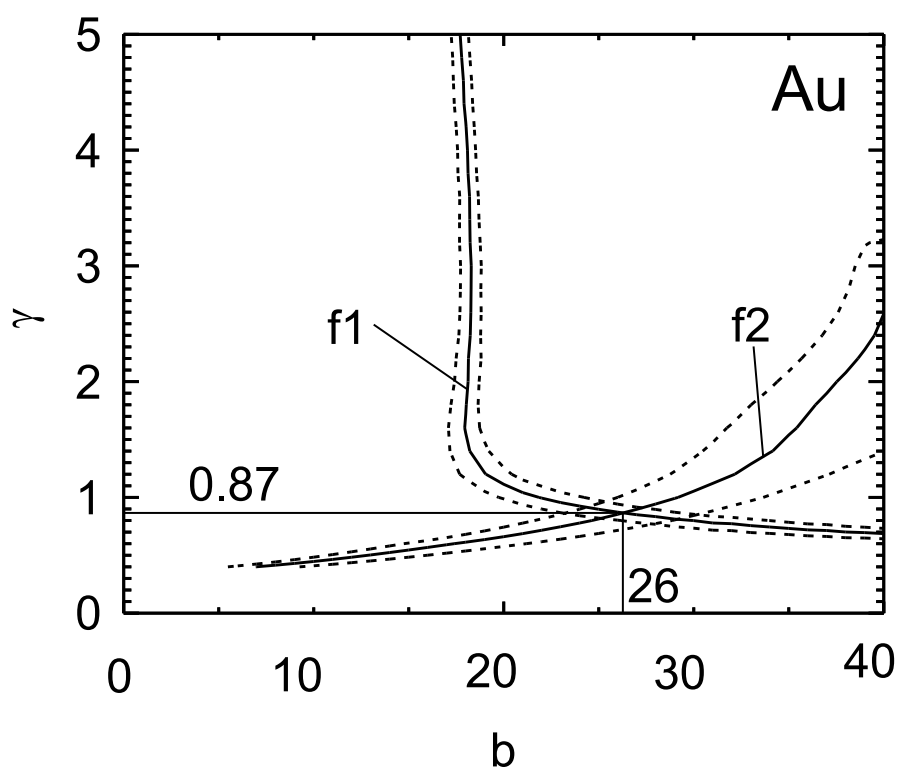

Abb. 4.12. Isokonzentrationslinien für die aus den experimentellen Daten bestimmten Parameter f1 und f2. Bei den gestrichelten Kurven ist f1 um $\pm 10 \%$ und $\mathrm{f} 2$ um $\pm 2 \%$ variiert. Am Schnittpunkt der beiden Kurven mit den Parametern $\mathrm{b}=26$ und $\gamma=0.87$ erfüllt der ermittelte Streuquerschnitt die experimentelle Vorgabe.

Das Ergebnis dieser Anpassung ist in Abbildung 4.10 und $4.13 \mathrm{zu}$ sehen. Die ermittelten Parameter b, g und $\Lambda$ der Streuquerschnitte für Kupfer und Gold sind in Tabelle 4.2 zusammengefaßt. Die Werte weichen aufgrund der optimalen Anpassung an den exponentiellen Abfall der Maximalintensität etwas von der in Abbildung 4.12 angegebenen Werten ab. Für Gold wurde der Streuquerschnitt und der Verlauf der Entwicklungskoeffizienten $a_{n}$ in Abbildung 4.7 und 4.8 aufgetragen. Der ermittelte Streuquerschnitt von Gold paßt gut zu den experimentell ermittelten normierten Streuquerschnitten unter kinematischen Bedingungen. Der experimentell ermittelte Streuquerschnitt hat außerdem große Ähnlichkeit mit dem Streuquerschnitt nach Wentzel, weicht jedoch bei größeren Streuwinkeln von diesem ab. Diese Abweichung ist nötig, um die erhöhte Streuung bei diesen 
4. Quantitative Auswertung der chemischen Analysetechniken

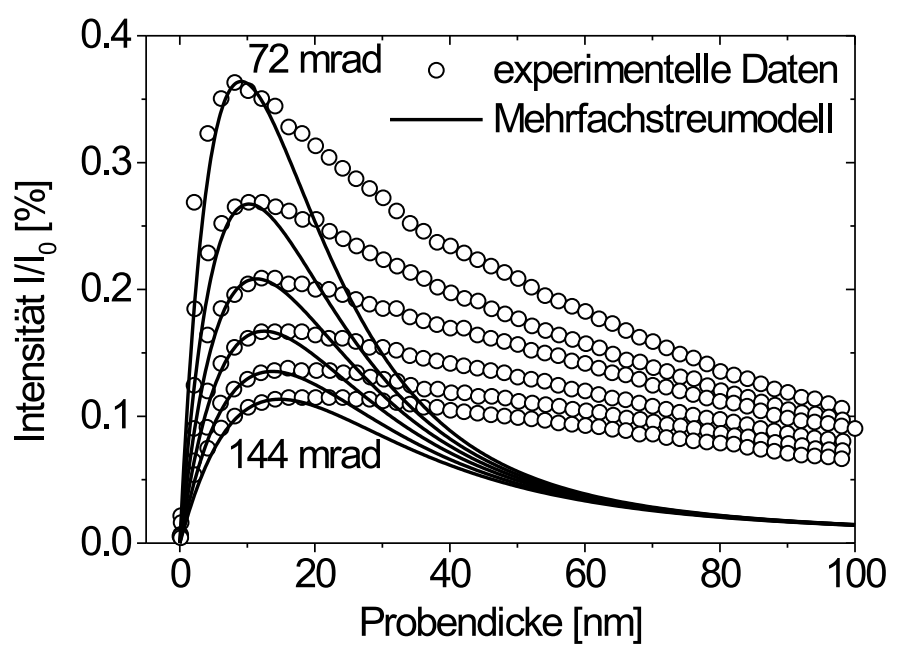

Abb. 4.13. Vergleich der nach dem Mehrfachstreumodell berechneten mit den experimentell ermittelten Intensitätswerten für Gold. Der dabei verwendete Streuquerschnitt ist nach dem in dieser Arbeit angegebenen Verfahren an die experimentellen Meßwerte angepaßt worden.

Winkeln beschreiben zu können. Die ermittelten freien Weglängen sind relativ klein, wobei die reskalierten Streuquerschnitte aus der Literatur noch kleinere Werte ergeben. Die für die verschiedenen Elemente aus den Intensitätsmaximas ermittelten Streuquerschnitte sind für Probendicken unterhalb des Maximums geeignet, die ermittelten Intensitätskurven zu beschreiben.

\subsubsection{Mehrkomponentige Systeme}

Da im Rahmen des Mehrfachstreumodells unabhängige Streuereignisse beschrieben werden, läßt sich das Modell sehr einfach auf mehrkomponentige Systeme erweitern. In einer Legierung ist die Wahrscheinlichkeit für ein Streuereignis an einem Atom einer Komponente proportional zum Atomanteil $\nu$. Damit ergibt sich der differentielle Streuquerschnitt einer Legierung aus N Elementen aus der gewichteten Summe der Streuquerschnitte der Elemente:

$$
\begin{aligned}
\sigma_{\text {tot }}^{\text {Leg }} & =\sum_{x=1}^{N} \nu_{x} \sigma_{\text {tot }}^{x} \\
\Lambda^{\text {Leg }} & =\frac{1}{N_{\nu}^{\text {Leg }} \cdot \sigma_{\text {tot }}^{\text {Leg }}} \\
S_{1}^{\text {Leg }}(\theta) & =\sum_{x=1}^{N} \frac{\nu_{x} \sigma_{\text {tot }}^{x}}{\sum_{y=1}^{N} \nu_{y} \sigma_{\text {tot }}^{y}} \cdot S_{1}^{x}(\theta)
\end{aligned}
$$

Damit können die Entwicklungskoeffizienten $a_{n}$ einer Legierung direkt aus den Koeffizienten der reinen Elemente berechnet werden: 


\section{Quantitative Auswertung der chemischen Analysetechniken}

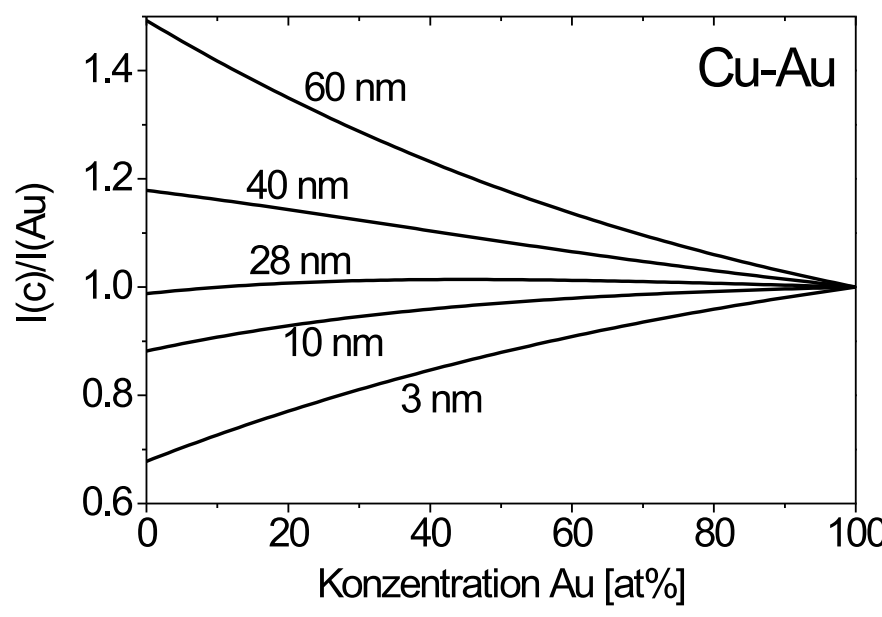

Abb. 4.14. Chemischer Kontrast aus dem inkohärenten Mehrfachstreumodell im System $\mathrm{Cu}-\mathrm{Au}$ für verschiedene Probendicken. Die Modellparameter wurden an die experimentellen Daten angepaßt.

$$
a_{n}^{\text {Leg }}=\sum_{x=1}^{N} \frac{\nu_{x} \sigma_{\text {tot }}^{x}}{\sum_{y=1}^{N} \nu_{y} \sigma_{t o t}^{y}} \cdot a_{n}^{x}
$$

Die Streuzentrendichte der Legierung muß zur Berechnung bekannt sein. Verhält sich die Gitterkonstante nach der Vegardschen Regel und geht man von einer nicht veränderten Struktur des Systems aus, so ergibt sich für eine Legierung:

$$
N^{L e g}=\frac{1}{\left(\sum_{x=1}^{N} \frac{\nu_{x}}{\sqrt[3]{N_{x}}}\right)^{3}}
$$

Eine besser Beschreibung als die Vegardsche Regel liefert der Ansatz nach Friedel [28], der die elastischen Eigenschaften des Systems berücksichtigt. Im allgemeinen Fall kann auch eine andere, dem betrachteten System angemessenere Beschreibung der Streuzentrendichte herangezogen werden. In Abbildung 4.14 ist eine Modellrechnung mit den angepaßten Parametern für das $\mathrm{Cu}-\mathrm{Au}$ System aufgeführt. Für kleine Probendicken steigt die Intensität mit zunehmendem Goldgehalt monoton an. Ab Probendicken von $30 \mathrm{~nm}$ ist eine Kontrastinversion zu verzeichnen, Kupfer liefert nun die höhere Elektronenintensität. Bei Probendicken im Bereich der Kontrastinversion liegt die Intensität von Mischkonzentrationen höher als die beider Elemente. Im Gegensatz zu Hellfeldaufnahmen findet bei der Z-Kontrastabbildung die Kontrastinversion nur einmal statt, was die Bildinterpretation erleichtert. Doch selbst bei kleinen Probendicken ist der Zusammenhang zwischen Konzentration und Intensität nicht linear. Die Z-Kontrastabbildung reagiert sehr viel empfindlicher auf kleine Goldkonzentrationen in einer Kupfermatrix verglichen mit kleinen Kupferkonzentrationen in einer Goldmatrix. Man findet somit keine einfache Beziehung zwischen der Intensität und der Konzentration.

Unter Kenntnis der Modellparameter läßt sich die Intensitätsfunktion I(c,t) in Abhängigkeit von der Konzentration und der Probendicke komplett berechnen. 


\section{Quantitative Auswertung der chemischen Analysetechniken}

Wenn eine der beiden Variablen bekannt ist, kann die andere durch Lösung der impliziten Gleichung $I_{\text {exp }}=I(c, t)$ bestimmt werden. Bei Probendicken unterhalb oder oberhalb der beiden Intensitätsmaxima ist die Lösung eindeutig. Dazwischen erhält man zwei Lösungen, wobei aufgrund der Randbedingungen in den meisten Fällen die richtige Lösung bestimmt werden kann. Um eine eindeutige Lösung, eine hohe Auflösung und einen großen Kontrast zu erhalten, sollten für eine quantitative Analyse möglichst dünne Probenstellen ausgewählt werden.

\subsubsection{Auswerteverfahren}

Nach den vorangegangenen Ausführungen über die Bildintensitäten stellt sich die Frage nach der praktischen Durchführung der chemischen Analyse. Aus einer elektronenmikroskopischen Aufnahme kann für jeden Punkt die lokale Elektronenintensität $I_{\text {exp }}$ bestimmt werden. Zur Bestimmung der lokalen Probenkonzentration durch Lösen der impliziten Gleichung $I_{e x p}=I(c, t)$ muß jedoch die Probendicke bekannt sein.

Ein mögliches Verfahren zur Bestimmung der Probendicke ist die Verwendung von Eichpunkten bekannter Konzentration außerhalb des Analysegebiets. An diesen Stellen kann mittels Lösung der impliziten Gleichung $I_{\text {exp }}=I\left(c_{\text {Eich }}, t\right)$ die Probendicke bestimmt werden. Von diesen Probenstellen aus wird die Probendicke in das Analysegebiet extrapoliert. Dort kann nun unter Verwendung der extrapolierten Probendicke die lokale Zusammensetzung ermittelt werden. Voraussetzung für die Anwendung dieses Verfahrens sind jedoch einen glattes Probenprofil und Eichpunkte bekannter Konzentration in der Nähe der Analyseregion. Für eine weitergehende Besprechung wird auf die Literatur verwiesen [38, 92].

\subsubsection{Genauigkeit der chemischen Analyse}

Die Anwendung einer Methode zur chemischen Analyse wirft die Frage nach ihrer räumlichen und chemischen Aufösung auf. In einer früheren Arbeit wurden von Schmitz $1 \mathrm{~nm}$ große, silberreiche Teilchen in einer Aluminium-Matrix mit einem scharfen Interface aufgelöst [91]. Zum anderen wurde von Hartung gezeigt, daß die Abbildung einer Grenzfläche mit einer Auflösung von besser als $2 \mathrm{~nm}$ möglich ist [38]. Die damit erreichte räumliche Auflösung entspricht damit denen der energiegefilterten Abbildungen mit aktuellen Geräten. Andererseits ist im STEM mit einem Ringdetektor eine atomare Auflösung erzielt worden. Nach dem Reziprozitätstheorem ${ }^{1}$ entspricht die kohärente Elektronenquelle des STEM der kohärenten Objektivlinse. Die Punktauflösung von 0.33 nm des verwendeten

\footnotetext{
${ }^{1}$ Auf das Konzept der Reziprozität zwischen Raster- und Transmissionselektronenmikroskop wird bei Schmitz [90] eingegangen.
} 


\section{Quantitative Auswertung der chemischen Analysetechniken}

Philips EM 420 ST Elektronenmikroskops kann damit nicht die Beschränkung der Aufösung auf einen Wert von etwa $1 \mathrm{~nm}$ erklären. Bis auf weiteres bleibt dieser Punkt ungeklärt.

Die Genauigkeit der chemischen Abbildung ist stark vom Streukontrast zwischen den beteiligten Elementen abhängig. Als eine erste Abschätzung kann die relative Differenz von $Z^{2}$ dienen, wie sie sich bei der nicht abgeschirmten Rutherford Streuung ergibt. Eine chemische Analyse mit der Z-Kontrastabbildung sollte möglich sein, wenn die Elemente in verschiedenen Reihen im Periodensystem stehen. Im folgenden werden einige quantitative Überlegungen zur Genauigkeit der chemischen Analyse in Kupfer-Gold, einem System mit mittlerem Kontrast, angestellt.

Die Intensität in der Z-Kontrastabbildung ist neben der Zusammensetzung noch von der Probendicke abhängig. Die Probendicke zwischen zwei Eichpunkten kann zufällig verursacht durch Rauhigkeiten oder systematisch durch bevorzugtes Ionendünnen während der Probenpräparation vom angenommenen Verlauf abweichen. Der zufällige Anteil wird aus der Messung an Eichstandards ermittelt. Hierbei zeigt sich, daß unter typischen Bedingungen eine Streuung von $\triangle I_{\text {noise }} / I \approx 2-3 \%$ beobachtet wird. Um den systematischen Fehler klein zu halten, werden nur Proben mit einem glatten Probenprofil verwendet. Bei einer Probe mit einer Abweichung durch selektives Ätzen von unter 10\%, was einer maximalen Dickenabweichung von $\triangle t / t<5 \%$ entspricht, ergibt sich ein Fehler bei der Bestimmung der Bildintensität von

$$
\frac{\triangle I}{I}=\sqrt{\left(\frac{\triangle I_{\text {noise }}}{I}\right)^{2}+\left(\frac{\triangle t}{t}\right)^{2}}=6 \% .
$$

Zusammen mit der Steigung der Intensitätskurve aus Abbildung 4.14 bei dünnen Probenstellen

$$
\left.I_{C u} \frac{d c}{d I}\right|_{C u} \approx 1.4 \quad,\left.I_{A u} \frac{d c}{d I}\right|_{A u} \approx 5
$$

ergibt sich der Fehler bei der chemischen Analyse zu

$$
\triangle c=I \cdot \frac{d c}{d I} \cdot \frac{\triangle I}{I}
$$

Im schlechtesten Fall folgt daraus ein Fehler in der Konzentrationsbestimmung von $8 \%$ für Kupfer und 30\% für Gold. Diese Werte können in der Regel deutlich unterschritten werden.

Selbst bei einem Kegelwinkel von 144 mrad waren noch meßbare Schwankungen der Bildintensität in Abbildung 4.2 in der Größenordnung von 5\% zu erkennen. Da das verwendete Kontrastmodell diese Orientierungsabhängigkeit nicht berücksichtigt, führen auch diese Schwankungen zu Fehlern bei der Konzentrationsbestimmungen in ähnlicher Größenordnung. 


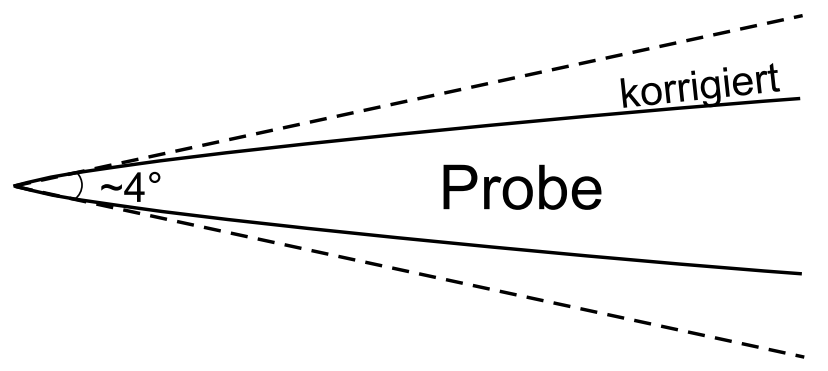

Abb. 4.15. Darstellung der Probenform der Goldeichprobe nach der Dickeneichung über Extinktionskonturen (gestrichelte Linie) und der hypothetischen Korrektur, die sich aus dem angepaßten Streuquerschnitt ergeben würde.

Zusätzlich muß man bei einer räumlich hochaufösenden Analyse das statistische Rauschen beachten. Ein Analysevolumen von $2 \times 2 \times 3 \mathrm{~nm}^{3}$ enthält nur noch $\mathrm{N}=$ 800 Atome, womit sich ein statistisches Rauschen von $\triangle c=\sqrt{c(1-c) / N}=2 a t \%$ ergibt.

\subsubsection{Diskussion}

Als Ergebnis ist festzuhalten, daß die HCDF Abbildung unter bestimmten Gegebenheiten die Bedingungen einer Z-Kontrastabbildung erfüllt. Das angegebene Mehrfachstreumodell ist bei geeignetem Streuquerschnitt in der Lage, die gemessenen Intensitätsprofile zu beschreiben. Die Übereinstimmung der damit errechneten Profile ist jedoch nicht so gut, um das Modell als endgültige Modellierung der Eigenschaften der HCDF Abbildung anzusehen.

Die schlechte Beschreibung der Beziehung zwischen Kegelwinkel und der zum Intensitätsmaximum gehörigen Probendicke in Abbildung 4.10 und die Abweichung der errechneten Intensitätskurven bei Probendicken hinter dem Intensitätsmaximum in Abbildung 4.13 ist besonders auffällig. Die Probendicke ist bei den verwendeten Eichkurven der Meßwert mit der größten Ungenauigkeit, da sie bei kleinen Probendicken aus dem Abstand der Extinktionskonturen in einer Zonenachsenorientierung bestimmt und zu größeren Probendicken extrapoliert wurde. Verfahren mit denen man die Probendicke z.B. über konvergente Elektronenbeugung [56] oder die Absorption einer charakteristischen Röntgenstrahlung [118] in einem größeren Dickenbereich gut bestimmen könnte, würden hier abhelfen. Nimmt man an, daß das Mehrfachstreumodell mit den ermittelten Streuquerschnitten bis zu großen Probendicken richtig ist, so kann man eine korrigierte Probenform berechnen. In Abbildung 4.15 wurde dies für die verwendete Goldeichprobe durchgeführt. Die resultierende Probenform ist durchaus glaubwürdig, zumal ein signifikantes Abflachen des Probenprofils erst im Bereich der extrapolierten Probendicke zu beobachten ist.

Zum anderen muß man in Betracht ziehen, daß das hier dargestellte Mehrfachstreumodell bei größeren Probendicken die experimentellen Daten prinzipiell nicht mehr beschreiben kann. Das Modell liefert nach Gleichung 4.7 bei einer beliebig 
4. Quantitative Auswertung der chemischen Analysetechniken

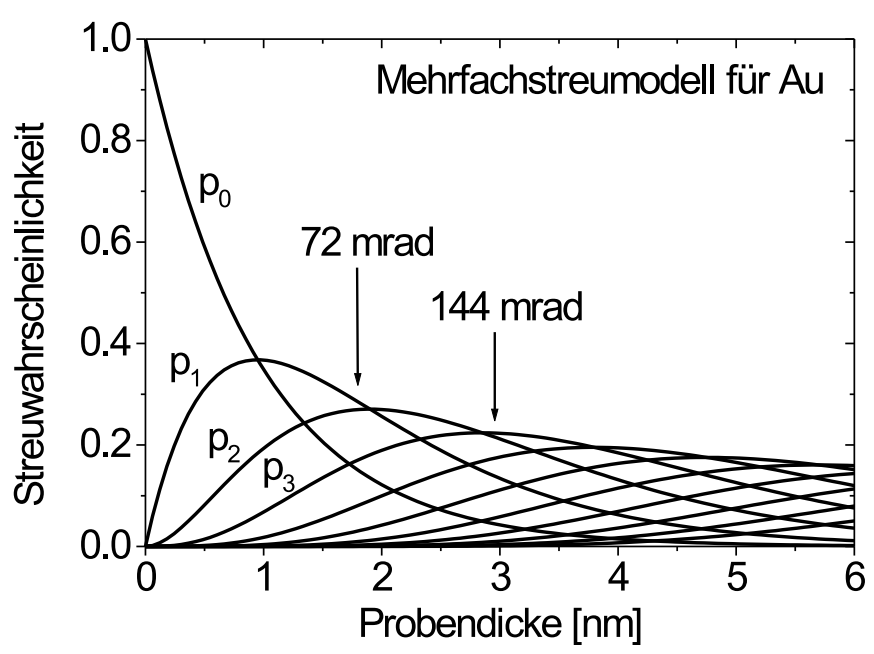

Abb. 4.16. Wahrscheinlichkeit $p_{n}$ eines n-fach Streuereignisses. Die Probendicke wurde über die mittlere freie Weglänge von $\mathrm{Au}$ festgelegt. Die Probendicken, bei denen das Intensitätsmaximum für verschiedene Kegelwinkel auftritt, sind mit Pfeilen bezeichnet.

dicken Probe eine konstante Intensität. Dies steht mit der Erfahrungstatsache in Konflikt, daß eine entsprechend dicke Probe für Elektronen undurchlässig ist. Das Elektron wird in diesem Fall abgebremst, von der Probe absorbiert und fließt über den Probenhalter ab. Verliert jedoch ein Elektron bei der Wechselwirkung mit der Probe Energie, ändert sich auch der zugehörige Streuquerschnitt und es würde sich bei den typischen Streuwinkeln der HCDF Abbildung eine erhöhte Intensität ergeben. Ein abgebremstes Elektron wechselwirkt stärker mit der Probe. Dies wird bei unseren Messungen z.B. in Abbildung 4.13 für Au im Vergleich zu den Rechnungen mit konstantem Streuquerschnitt auch beobachtet. Damit ergibt sich der Streuquerschnitt nach n Stößen $S_{n}$ nicht durch n-fache Faltung des Einfachstreuquerschnitts $S_{1}$, sondern müßte entsprechend modifiziert werden. Um hier Klarheit zu schaffen sind grundlegende Rechnungen zum Streuprozeß bei der HCDF Abbildung notwendig.

Um die Grenzen des Mehrfachstreumodells bestimmen zu können, betrachtet man die Verteilung der Anzahl der Streuprozesse bei unterschiedlichen Probendicken. Diese ist in Abbildung 4.16 für Au aufgetragen. Bei kleinen Probendicken bis zum Intensitätsmaximum wird der größte Teil der Bildintensität durch Elektronen geliefert, die an nur wenigen Streuprozessen beteiligt waren. Dabei sollte sich der Energieverlust der Elektronen in Grenzen halten. Damit ist es für Probendicken unterhalb des Intensitätsmaximums gerechtfertigt, den Streuquerschnitt eines mehrfachen Streuereignisses durch Faltung aus dem einfachen Streuquerschnitt zu ermitteln und die Bildauswertung mit dem dargestellten Mehrfachstreumodell durchzuführen. Der Kontrast der Abbildung bei Annäherung an das erste Intensitätsmaximum bei einer Legierung wird deutlich schlechter, wie man in Abbildung 4.14 nachvollziehen kann. Bei noch größeren Probendicken ist ohnehin die räumliche Auflösung dann soweit reduziert [38], daß eine Analyse nicht mehr sinnvoll erscheint. Die oben dargestellten Ergebnisse zeigen, daß HCDF 


\section{Quantitative Auswertung der chemischen Analysetechniken}

Elektronenmikroskopie unter den angegebenen Bedingungen geeignet ist, chemische Information mit einer hohen Ortsaufösung zu erhalten. So kann die HCDF Abbildung entscheidende Informationen liefern, um die untersuchte Interdiffusion $\mathrm{zu}$ verstehen.

\subsection{Sekundärneutralteilchenmassenspektrometrie}

Zur quantitativen Auswertung der SNMS-Messungen ist es notwendig, die experimentell ermittelten Intensitätsmeßwerte in Konzentrationen und die Sputterzeit in eine Tiefenskala umzurechnen. Die allgemeine Vorgehensweise und die auftretenden Probleme werden stellvertretend am Beispiel eines Kupfer-Gold Schichtsystems dargestellt. Die weiteren untersuchten Schichtsysteme wurden auf gleiche Weise behandelt. Die entsprechenden Daten werden im Anhang angegeben.

In Abbildung 4.17 ist eine typische SNMS Messung einer ausgelagerten $\mathrm{Cu}-\mathrm{Au}$ Schichtprobe mit einer Schichtdicke von jeweils $60 \mathrm{~nm}$ aufgetragen. Die einzelnen Elemente weisen eine unterschiedliche Sättigungsintensität auf. Auch die Zeit zum Abtragen der einzelnen Schichten unterscheidet sich aufgrund der verschiedenen Sputterraten.

Um eine Analyse der Meßdaten vornehmen zu können, ist ein genaueres Bild der einzelnen, sich wiederholenden Schritte während einer Messung nötig:

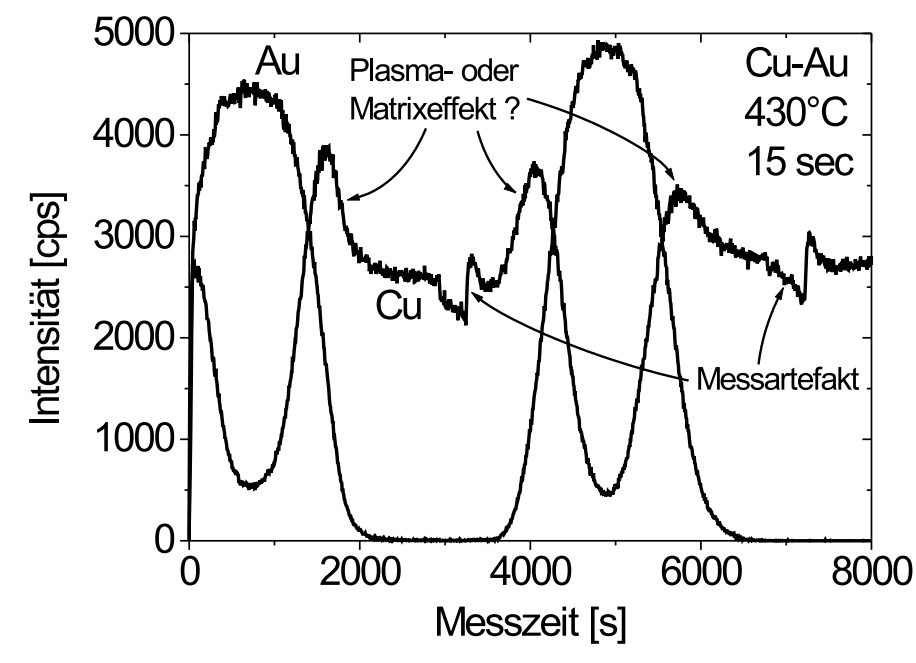

Abb. 4.17. Intensität der analysierten Kupfer- und Goldatome in Abhängigkeit von der Meßzeit für ein bei $430{ }^{\circ} \mathrm{C}$ für 15 Sekunden ausgelagertes $\mathrm{Cu}-$ $\mathrm{Au}$ Schichtpaket mit $60 \mathrm{~nm}$ Schichtdicke. Die Sättigungsintensitäten von Kupfer und Gold unterscheiden sich erheblich. Auch erscheint die Kupferschicht breiter als die Goldschicht. An der durchmischten Grenzfläche ist das Kupfersignal deutlich erhöht. Die Meßartefakte resultierten aus Schwankungen der Laserintensität. 


\section{Quantitative Auswertung der chemischen Analysetechniken}

1. Mit einer Analysequelle (Gallium oder Cäsium) wird Probenmaterial abgetragen. Die geladenen Teilchen werden ionenoptisch unterdrückt, um den Matrix-Effekt der Sekundärionenemission auszuschließen. Nur die Neutralteilchen bleiben zurück. Für die Analysequelle hat sich eine hohe Energie der Primärionen bei sehr kleinem Strom bewährt, um eine möglichst konzentrationsunabhängige Teilchenausbeute zu erhalten.

2. Nach einer kurzen Zeit werden die verbleibenden Neutralteilchen vom Laser ionisiert und vom Flugzeitmassenspektrometer analysiert.

3. Eine dünne Schicht der Probe wird mit der Argonquelle abgetragen. Die Sputterquelle wird mit einer möglichst kleinen Beschleunigungsspannung betrieben, um das Durchmischen der Probe zu verhindern.

Man sieht, daß die Kenntnis der beim Probenabtrag unter Ionenbeschuß herrschenden Bedingungen für die Auswertung wichtig ist. Um diese zu bestimmen wurden TRIM-Simulationen der beiden Abtragprozesse für die jeweiligen Schichtsysteme durchgeführt.

\subsubsection{TRIM-Simulation}

Das Programm TRIM wurde von Ziegler et al. [125] entwickelt, um die Eindringtiefe und das Schädigungsverhalten von Ionen zu berechnen. Dazu wird mit Hilfe der Monte-Carlo-Technik ein einfallendes Ion, daß sich durch das Material bewegt, simuliert. Bei der Bewegung durch das Material verliert das Ion durch Stöße an Energie. Das Programm betrachtet die Probe als amorph und berücksichtigt nur Zweiteilchenstöße bei einer Temperatur von $0 \mathrm{~K}$.

Ist die an ein Atom übertragene Energie größer als die Verlagerungsenergie, wird auch die Bewegung des getroffenen Teilchens durch das Material verfolgt. So wird eine komplette Stoßkaskade für ein einfallendes Ion simuliert und die Parameter wie Ort, Art der Wechselwirkung, Stoßpartner und Energieverlust werden protokolliert. Zur Gewinnung der gesuchten Kenngrößen wie z.B. der Sputterrate muß eine genügend große Anzahl von Simulationsschritten durchgeführt werden. Bei den hier gezeigten TRIM-Simulationen wurden für die $25 \mathrm{keV}$ Galliumionen 10000 Ionen, für die $700 \mathrm{eV}$ Argonionen jeweils 40000 Ionen simuliert, um eine gute Statistik zu erhalten. Aus den Simulationen wurden die pro eingeschossenem Ion die Probe verlassenden Atome und deren mittlere Energie bestimmt. 


\section{Quantitative Auswertung der chemischen Analysetechniken}

\subsubsection{Kalibrierung der Meßtiefe}

Aus den TRIM-Simulationen wurde die Abtragrate verschiedener Legierungen unter dem Beschuß mit $700 \mathrm{eV}$ Argonionen in einem $45^{\circ}$ Einfallswinkel bestimmt. Das Ergebnis dieser Simulation für Kupfer-Gold findet sich in Abbildung 4.18. Die Abtragrate entspricht dabei der Änderung der Schichtdicke dx/dt. Die geringe Abhängigkeit der totalen Ausbeute von der Konzentration findet sich auch bei ähnlichen Abtragbedingungen in der Literatur [7]. Dies wird auch durch die Tatsache bestärkt, daß die Zeit zum Abtragen des kompletten Schichtpakets unabhängig von seinem Auslagerungszustand und damit der Konzentrationsverteilung innerhalb der Probe ist. Die geringen Abweichungen der partiellen Ausbeute von der stöchiometrischen Zusammensetzung kann zu einer geringen Anreicherung von Gold an der Oberfläche nach dem Abtrag mit Argon führen. In der Literatur findet man im Rahmen der experimentellen Genauigkeit ein stöchiometrisches Verhältnis der abgetragenen Atome [84,61]. Auch wurde keine Anreicherung der Oberfläche mit Kupfer oder Gold gefunden [7,23,110], womit keine direkte Beeinflussung der Analyse durch den Abtragprozeß zu erwarten ist.

Der gefundene Zusammenhang zwischen Konzentration und Abtragrate aus Abbildung 4.18 wurde mit einem Polynom 3. Grades angepaßt und zur Auswertung verwendet. Aufgrund der für einen Datenpunkt ermittelten Konzentration wurde die Zeitachse entsprechend der Abtragrate gestreckt, wodurch eine zur Tiefe proportionale Zeitskala entsteht. Zur absoluten Eichung der Dickenskala wurde die so korrigierte Zeitachse gestaucht, so daß die Stoffmenge $\int_{0}^{\infty} c_{A u}(x) d x$ einer
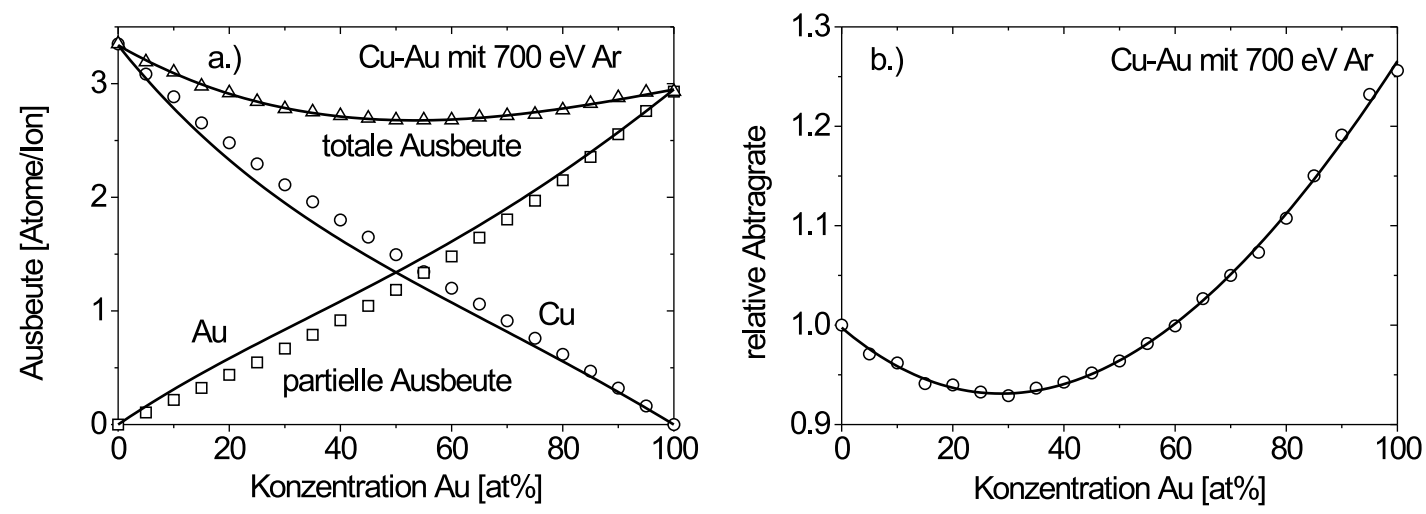

Abb. 4.18. Durch TRIM-Simulationen wurde (a) die totale und partielle Ausbeute und (b) die relative Abtragrate von Kupfer-Gold Legierungen im Vergleich zu reinem Kupfer unter dem Beschuß mit $700 \mathrm{eV}$ Argonionen unter einem Einfallswinkel von $45^{\circ}$ bestimmt. Die durchgezogene Linie in (a) kennzeichnet das stöchiometrische Verhältnis aus der totalen Ausbeute. Die Abtragrate entspricht der Änderung der Schichtdicke dx/dt. Die relative Abtragrate wurde auf den Abtrag von reinem Kupfer normiert. 


\section{Quantitative Auswertung der chemischen Analysetechniken}

Komponente einen konstanten Wert annahm. Dieser wurde aus dem bekannten Schichtdickenprofil ermittelt. Die Dicke der Schichten wurde aus elektronenmikroskopischen Untersuchungen einer querschnittspräparierten Probe oder aus Messungen der Schichtdicke mit einem Schichtdickenmeßgerät an unter gleichen Bedingungen hergestellten Eichproben ermittelt.

\subsubsection{Konzentrationsbestimmung}

Da zur Analyse nicht die Argonsputterquelle, sondern eine Galliumionenquelle eingesetzt wird, unterscheiden sich die Bedingungen im Vergleich zum Sputtern. Für eine quantitative Konzentrationsbestimmung muß nun eine Beziehung zwischen den gemessenen Intensitäten und der Konzentration gefunden werden. Ein in der Literatur gängiger Ansatz ist lineare Interpolation zwischen ausgemessenen Eichsubstanzen [48, 108,94]. Im Fall eines Schichtpakets können dazu die einzelnen Schichten im unreagierten Ausgangszustand herangezogen werden. Die Konzentration ergibt sich dann wie folgt:

$$
c_{x}^{*}=\frac{\frac{I_{x}}{I_{x}^{M a x}}}{\sum_{n} \frac{I_{n}}{I_{n}^{M a x}}}
$$

wobei $I_{x}$ der gemessenen Intensität des Elements x in der Legierung und $I_{x}^{M a x}$ die Intensität der Reinkomponente x entspricht. Für $\mathrm{Cu}$-Au findet man ein Verhältnis zwischen der maximalen Kupfer- und Goldintensität von 1 zu 2. Das Verhältnis der Intensitäten zwischen zwei Elementen wird auch als relativer Empfindlichkeitsfaktor S(A,B) bezeichnet und kann zusätzlich aus Eichmessungen nach folgender Beziehung bestimmt werden:

$$
S(A, B)=\frac{I_{A}}{I_{B}} \cdot \frac{c_{B}}{c_{A}}=\frac{I_{A}^{M a x}}{I_{B}^{M a x}}
$$

mit $I_{A}$ und $I_{B}$ den gemessenen Intensitäten einer Legierung mit der Konzentration $c_{A}$ und $c_{B}$. Relative Empfindlichkeitsfaktoren für verschiedene Systeme sind zum Beispiel in [48] angegeben.

Aus den TRIM-Simulationen ergeben sich für verschiedene Legierungen die in Abbildung 4.19 dargestellten Ausbeuten. Die Abweichung von dem durch die Legierung vorgegebenen stöchiometrischen Verhältnis ist relativ klein. Der Ansatz nach Gleichung 4.18 wird durch die TRIM Rechnungen gestützt und sollte die Meßwerte beschreiben, sofern die Ionisierungswahrscheinlichkeit durch die Lasernachionisation für das einzelne Element unabhängig von der Konzentration ist. 

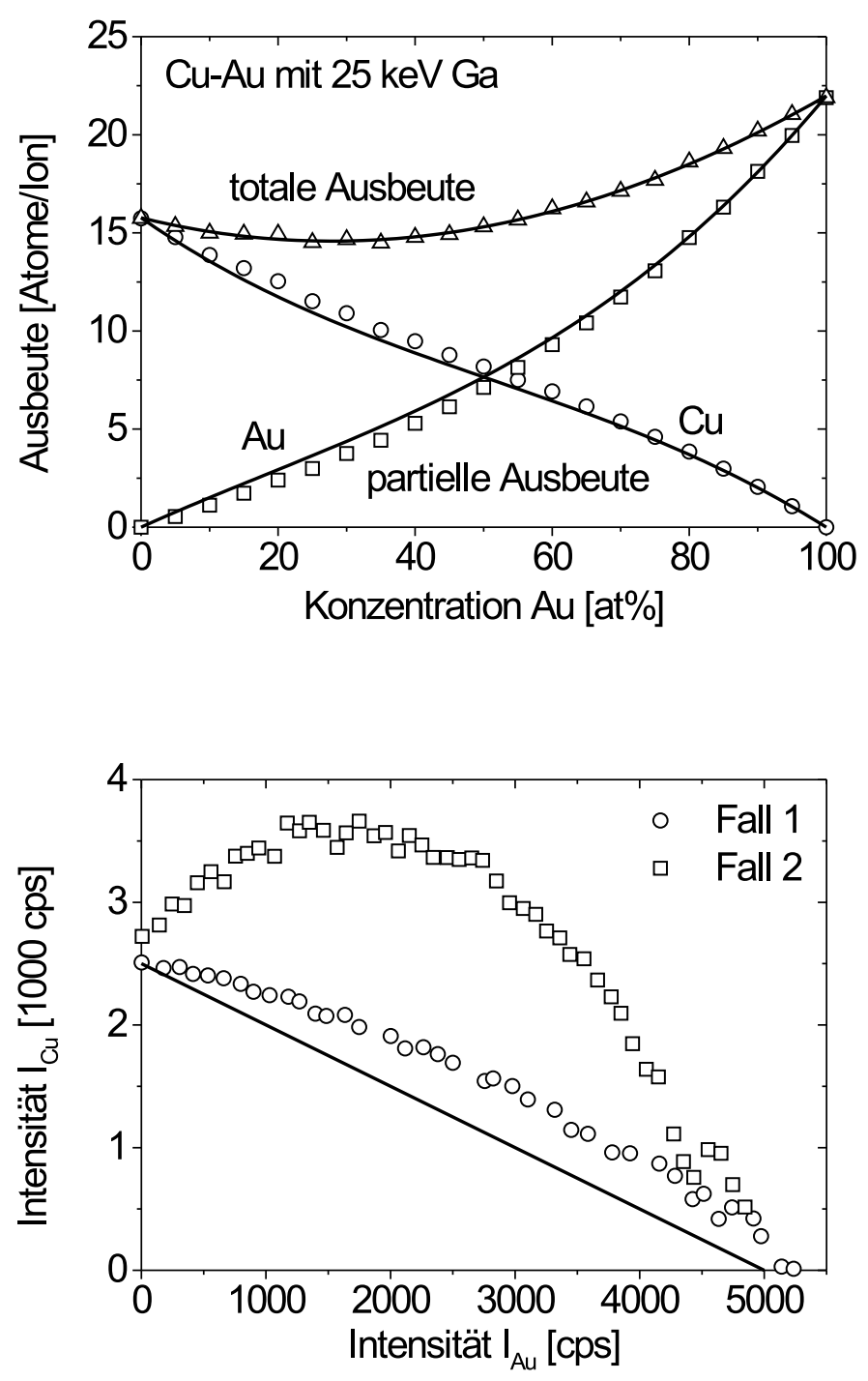

Abb. 4.19. Durch TRIMSimulationen wurde die Ausbeute von Kupfer-Gold Legierungen unter dem Beschuß mit $25 \mathrm{keV}$ Galliumionen unter einem Einfallswinkel von $45^{\circ}$ bestimmt. Die Simulationswerte der totalen Ausbeute wurden mit der durchgezogenen Kurve angepaßt. Die durchgezogenen Linien bei den partiellen Ausbeuten entsprechen jeweils einem stöchiometrischen Verhältnis unter Verwendung der simulierten totalen Ausbeute.

Abb. 4.20. Darstellung der korrespondierenden Intensitätswerte von Kupfer und Gold, die während einer SNMS Messung auftreten. Fall 1 entspricht der Messung einer scharfen Grenzfläche zwischen zwei Schichten, Fall 2 dem kontinuierlichen Übergang von Kupfer nach Gold in einem flachen Konzentrationsgradienten. Die durchgezogene Linie würde sich aus einem linearen Modell nach Gleichung 4.18 ergeben.

Um den Ansatz zu überprüfen wurden die korrespondierenden Intensitäten von Kupfer und Gold einer SNMS Messung in Abbildung 4.20 dargestellt. Den linearen Ansatz, der zu der durchgezogenen Linie führen würde, erfüllt jedoch nur der Fall 1 annähernd. Wie in Abbildung 4.21 gezeigt, liegen in diesem Fall die Elemente räumlich getrennt vor. Nur in einem kleinen Bereich der Analysefläche befindet sich eine Legierung. Dieser Fall wird jedoch unabhängig vom Verhalten einer Legierung immer einem linearen Modell gehorchen. Im Fall 2 liegt, wie in Abbildung 4.21 angedeutet, im Analysebereich eine nahezu homogene Konzentrationsverteilung vor. Der Fall 2 entspricht der Messung einer homogenen Legierung und der Verlauf der Kurve muß in Abhängigkeit von der Konzentration bestimmt werden. 
Fall 1

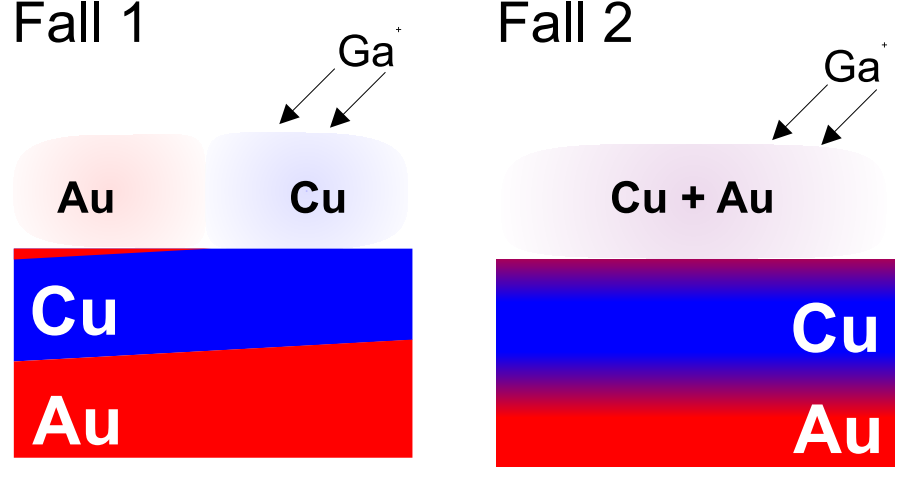

Abb. 4.21. Schematische Darstellung der Abtragbedingungen bei einer SNMS Messung für eine leicht schiefliegende, aber scharfe Grenzfläche (Fall 1) und bei einem flachen Konzentrationsgradienten (Fall 2).

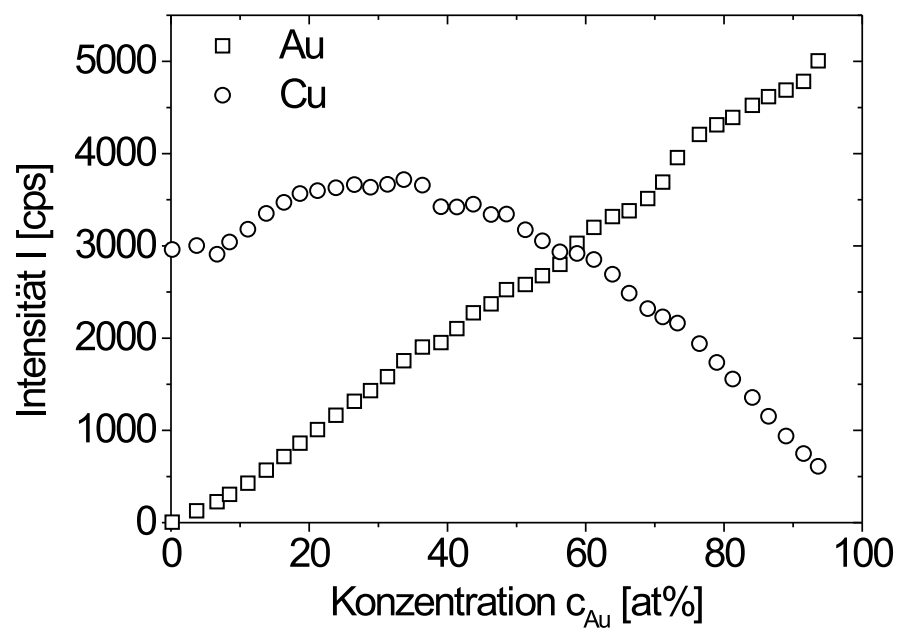

Abb. 4.22. Übertragungsfunktion zwischen der Probenkonzentration und der daraus resultierenden Intensitätswerte im $\mathrm{Cu}-\mathrm{Au}$ System. Die Schwankung der Werte ist auf die Verwendung von experimentellen Daten zurückzuführen.

Da während der Messungen absolute Schwankungen der Intensitäten auftraten, konnten für die Analyse keine Absolutwerte verwendet werden. Als Ausgangspunkt für die Auswertung wurde die Gleichung 4.18 verwendet. Die Konzentration wurde jedoch durch eine einfache, glatte Übertragungsfunktion korrigiert. Die Übertragungsfunktion wurde aus den Messungen ein und derselben Probe in verschiedenen Auslagerungszuständen ermittelt, so daß die Grenzen der einzelnen Schichtpakete für alle Proben nach der Kalibrierung der Meßtiefe wieder aufeinanderfallen. Die entsprechende Auftragung ist in Abbildung $6.8 \mathrm{zu}$ finden. Da die Proben eine unterschiedliche Konzentrationsverteilung aufweisen, ergibt sich eine implizite Eichung des kompletten Konzentrationsbereichs. Die sich daraus abgeleitete Übertragungsfunktion zwischen der Probenkonzentration und der per SNMS gemessenen Intensität ist in Abbildung 4.22 dargestellt.

Damit ergibt sich ein systematischer Fehler bei unterschiedlichen Konzentrationen im Analysegebiet, die sich aus einer inhomogenen Konzentrationsverteilung in der Probe oder einem steilen Konzentrationsgradienten ergeben. Um systematische Fehler zu vermeiden, wurden die ermittelten korrespondierenden Intensitäten der beteiligten Elemente für jede Messung aufgetragen. Diese mußten auf der in Abbildung 4.20 dargestellten Spur für den Fall 2 liegen, eine Abweichung deutet auf 


\section{Quantitative Auswertung der chemischen Analysetechniken}

eine inhomogene Konzentrationsverteilung hin und verhindert eine Analyse nach dem oben beschriebenen Verfahren.

\subsubsection{Einfluß von Grenzflächen}

Aus den Eichkurven in Abbildung 4.22 ist zu entnehmen, daß eine kupferreiche Legierung eine erhöhte Kupferintensität zur Folge hat. Bei der Messung einer unreagierten Kupfer-Gold Schichtprobe, die in Abbildung 4.23 dargestellt ist, findet man eine solche Überhöhung der Kupferintensität an der Grenzfläche von Kupfer nach Gold. Es stellt sich die Frage, ob die hergestellte Grenzfläche von Kupfer zum Gold eine durch die Herstellung bedingte Durchmischung aufweist, die Analyse diese Durchmischung verursacht oder die Überhöhung der Intensität mit der Grenzfläche der beiden Materialien an sich zusammenhängt.

Dazu wurde in einer TRIM-Simulation die Messung durch eine scharfe Grenzfläche berechnet. Die Dicke einer Decklage wurde systematisch verringert und die Ausbeute beim Beschuß mit $25 \mathrm{keV}$ Galliumionen berechnet. Dies ist in Abbildung 4.24a und 4.24d dargestellt. Dabei nimmt die Ausbeute an Goldatomen beim Verringern der Dicke einer Kupferschicht auf einer Goldschicht systematisch ab etwa $4 \mathrm{~nm}$ ab und das Kupfersignal steigt ab etwa $1 \mathrm{~nm}$ Dicke an. Im umgekehrten Fall einer Kupferschicht auf einer Goldschicht steigt bei Annäherung an die Grenzfläche die Kupferintensität ab $4 \mathrm{~nm}$ Schichtdicke an, um nach einem Maximum bei $0.8 \mathrm{~nm}$ endgültig abzufallen. Die Goldintensität steigt ab einer verbleibenden Schichtdicke von $1 \mathrm{~nm}$ monoton an. Um dies zu verstehen, hilft ein Blick auf die Energien der austretenden Atome in Abbildung 4.24c und 4.24d. In beiden Fällen nimmt die Energie der aus der tieferen Schicht herausgelösten Atome monoton ab. Die Atome mit niedrigerer Energie werden absorbiert und lösen andere Atom heraus. Im Fall einer Golddecklage werden durch die aus den

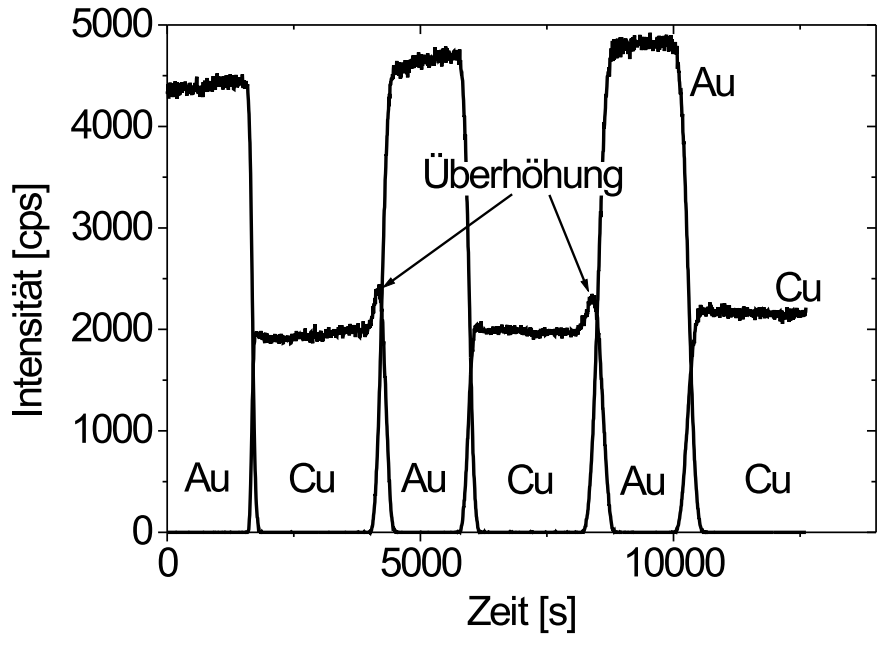

Abb. 4.23. Rohdaten einer $\mathrm{Cu}-\mathrm{Au}$ Schichtprobe mit einer Schichtdicke von jeweils $60 \mathrm{~nm}$. Die maximalen Intensitäten von Kupfer und Gold unterscheiden sich wesentlich. Weiter ist eine Erhöhung des Kupfersignals an der Grenzfläche vom $\mathrm{Cu}$ zum $\mathrm{Au}$ zu finden. 


\section{Quantitative Auswertung der chemischen Analysetechniken}

tieferen Schichten ausgelösten leichten Kupferionen weniger schwere Goldatome aus der Decklage herausgelöst. Im umgekehrten Fall einer Kupferdecklage werden durch die schweren Goldatome mehr leichte Kupferionen aus der Decklage herausgelöst als dies in den reinen Materialien möglich wäre. Die Darstellung von jeweils 100 in eine Grenzfläche mit einer $1 \mathrm{~nm}$ dicken Deckschicht geschossenen Ionen in Abbildung 4.24c und 4.24f illustriert das Simulationsergebnis noch einmal.

Die Erhöhung der Kupferintensität hängt damit direkt mit der Anwesenheit der Grenzfläche und dem tiefen Eindringen der Galliumionen in das Material zusammen.

\subsubsection{Diskussion}

Die chemische Analyse mit der SNMS ist an einige Bedingungen geknüpft. Man findet einen nichtlinearen Zusammenhang zwischen den gemessenen Intensitäten und der Konzentration. Damit ergibt sich die Bedingung, daß die analysierte Schicht eine homogene Konzentrationsverteilung aufweisen muß. In allen anderen Fällen liefert die chemische Analyse systematisch falsche Konzentrationen. Dies ist auch der Fall für einen steilen Konzentrationsgradienten, bei dem an verschiedenen Analysestellen stark unterschiedliche Konzentrationen vorliegen können. Selbst eine einfache, scharfe Grenzfläche liefert ein kompliziertes Intensitätsprofil, aus dem sich das Konzentrationsprofil der untersuchten Probe nicht direkt erschließt. Bei der Bewertung der Meßergebnisse muß dies berücksichtigt werden.

Abschließend bleibt die Frage nach dem Grund für die deutliche Erhöhung des Kupfersignals bei Mischlegierungen um die 30 at\% Gold aus Abbildung 4.20. Nach den Beobachtungen an dünnen Kupferschichten auf Gold könnte dafür eine Anreicherung von Kupfer auf der goldreichen Legierung durch den Probenabtrag mit der Argonquelle in Frage kommen. Dagegen spricht aber, daß eine solche Anreicherung in der Literatur nicht gefunden wird. Damit bleibt nur der Ionisierungsprozeß als Quelle des überhöhten Kupfersignals übrig. Befindet sich die Ionisierung der Neutralteilchen in der Sättigung, so dürfte keine Steigerung des Kupfersignals gegenüber dem Signal des reinen Kupfers mehr möglich sein. Ein weiterer Hinweis darauf, daß das Ionisierungsverhalten der Neutralteilchen nicht in der Sättigung ist, ergibt sich aus der empfindlichen Abhängigkeit der gemessenen Intensitäten von der Justierung des Laserstrahls. Offensichtlich können die schon ionisierten Goldatome die Ionisation weiterer Kupferatome in einem Mehrteilchenprozeß unterstützen. Für die detaillierte Aufklärung dieses Effekts reichen die vorhandenen Daten jedoch nicht aus.

Abschließend ist zu sagen, daß eine quantitative Analyse mit der SNMS mit der dargelegten Vorgehensweise gut möglich ist. Als Alternative zur Untersuchung 
4. Quantitative Auswertung der chemischen Analysetechniken
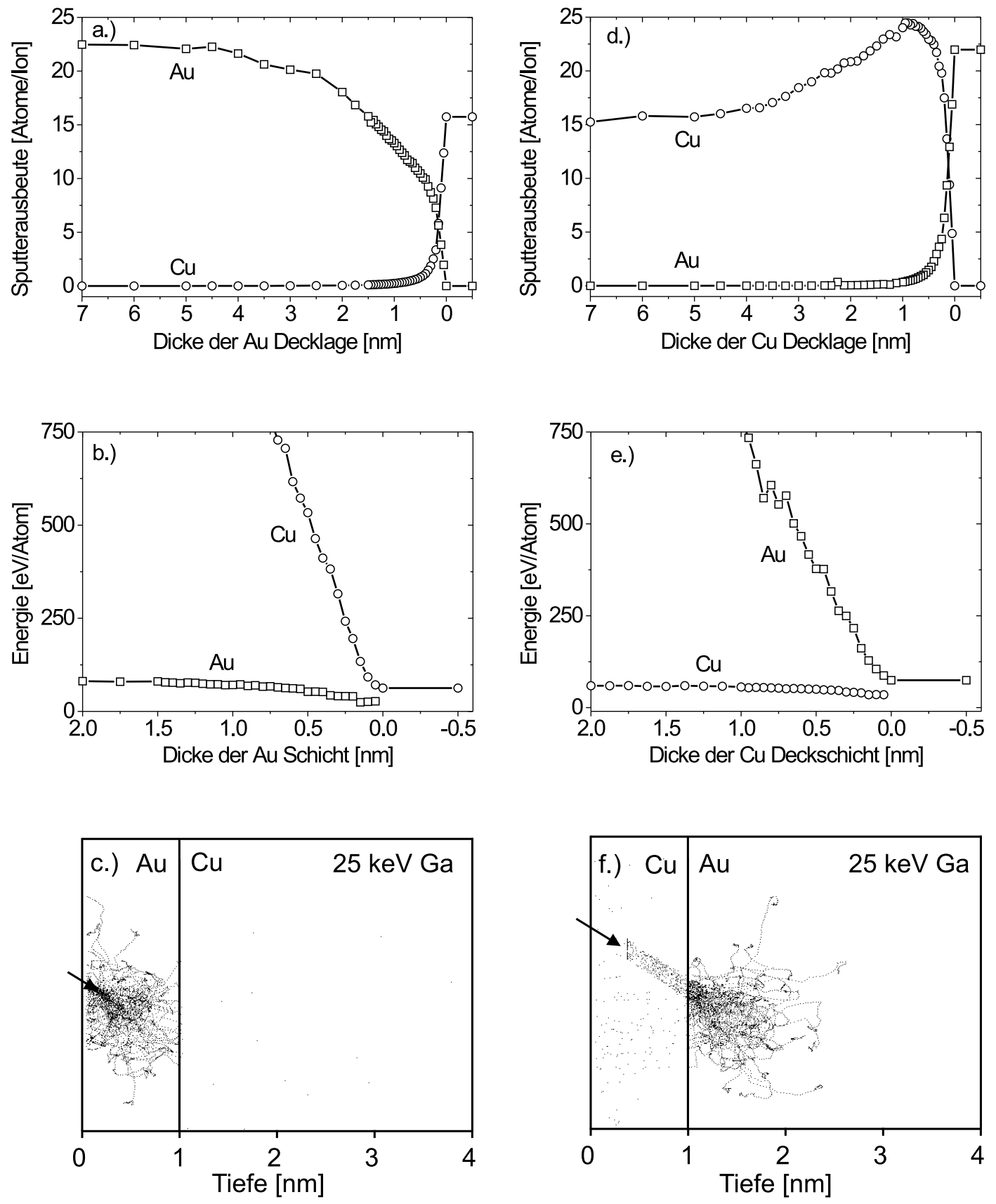

\begin{abstract}
Abb. 4.24. TRIM-Simulation einer SNMS Messung mit $25 \mathrm{keV}$ Galliumionen durch eine scharfe Grenzfläche vom Gold ins Kupfer (a-c) und vom Kupfer ins Gold (d-f). In (a) und (d) sind die Sputterausbeuten, in (b) und (e) die Energien der ausgelösten Atome in Abhängigkeit von der Dicke der verbleibenden Deckschicht aufgetragen. In (c) und (f) sind jeweils Bahnen von 100 Galliumionen aufgetragen, die auf ein Schichtsystem mit einer $1 \mathrm{~nm}$ dicken Deckschicht geschossen wurden.
\end{abstract}


4. Quantitative Auswertung der chemischen Analysetechniken

von Eichstandards hat sich die implizite Eichung durch verschiedene Auslagerungszustände einer Probe bewährt. Man sollte für die untersuchten Systeme jedoch die Notwendigkeit einer nichtlineare Korrektur der Intensitätswerte, wie sie in dieser Arbeit durchgeführt wurden, überprüfen. 


\section{Charakterisierung der Schichtsysteme}

In diesem Kapitel werden die Ergebnisse der Charakterisierung der verschiedenen Schichtsysteme vorgestellt. Dabei wird speziell auf den Einfluß der einkristallinen Metallsubstrate eingegangen.

\subsection{Silber/Gold}

Da die beiden Elemente Silber und Gold eine kubisch-flächenzentrierte Struktur aufweisen und nur ein sehr geringer Gittermisfit vorhanden ist, kann man unter geeigneten Herstellungsbedingungen eine direkte Beibehaltung der Struktur über die Grenzfläche hinweg erwarten. Beim epitaktischen Schichtwachstum einer Schicht auf einem Substrat mit anderer Gitterkonstante müssen die Atome an der Grenzfläche ihre Positionen so anpassen, daß der Gittermisfit ausgeglichen wird. Theoretische Untersuchungen dieses Problems wurden zuerst von Frank und van der Merwe durchgeführt [27]. Die Modelle wurden von vielen anderen Autoren weiterentwickelt $[114,43,4,5]$. Eine allgemeine Zusammenfassung ist bei Nix zu finden [73]. Die Modelle beschreiben, wie der natürliche Misfit $f_{0}$ durch das Substrat und die aufgetragene Schicht relaxiert wird. Der natürliche Misfit $f_{0}$ ist dabei gegeben durch

$$
f_{0}=\frac{a_{s}^{0}-a_{l}^{0}}{a_{l}^{0}}
$$

mit $a_{s}^{0}$ und $a_{l}^{0}$ der spannungsfreie Gitterparameter des Substrats und der aufgetragenen Schicht. Aus diesen Modellen geht hervor, daß die ersten Schichten bis zu einer kritischen Schichtdicke $h_{k}$ kohärent auf dem Substrat aufwachsen. Für größere Schichtdicken ist es energetisch vorteilhafter Misfitversetzungen einzubauen. Die Zahl der eingebauten Versetzungen, die die elastischen Spannungen relaxieren, strebt mit zunehmender Schichtdicke gegen einen Gleichgewichtswert.

Aus der (111) Polfigur, die in Abbildung 5.1 aufgetragen wurde, kann man eine epitaktische Schichtstruktur erkennen. Man findet eine direkte Übertragung der (111) Gitterebenen des Einkristalls (a-c), die man auch in einer (111) Polfigur des Substrats ohne Schichten findet, auf die Schicht. Die Ebenenschar (d), die man nur in den beschichteten Substraten findet, weist dagegen einen Winkel von $55^{\circ} \mathrm{zu}$ den (111) Ebenen (b) und (c) auf, der zwischen (200) und (111) Ebenen auftritt. Bei einer solchen Konstellation handelt es sich um Zwillinge. 


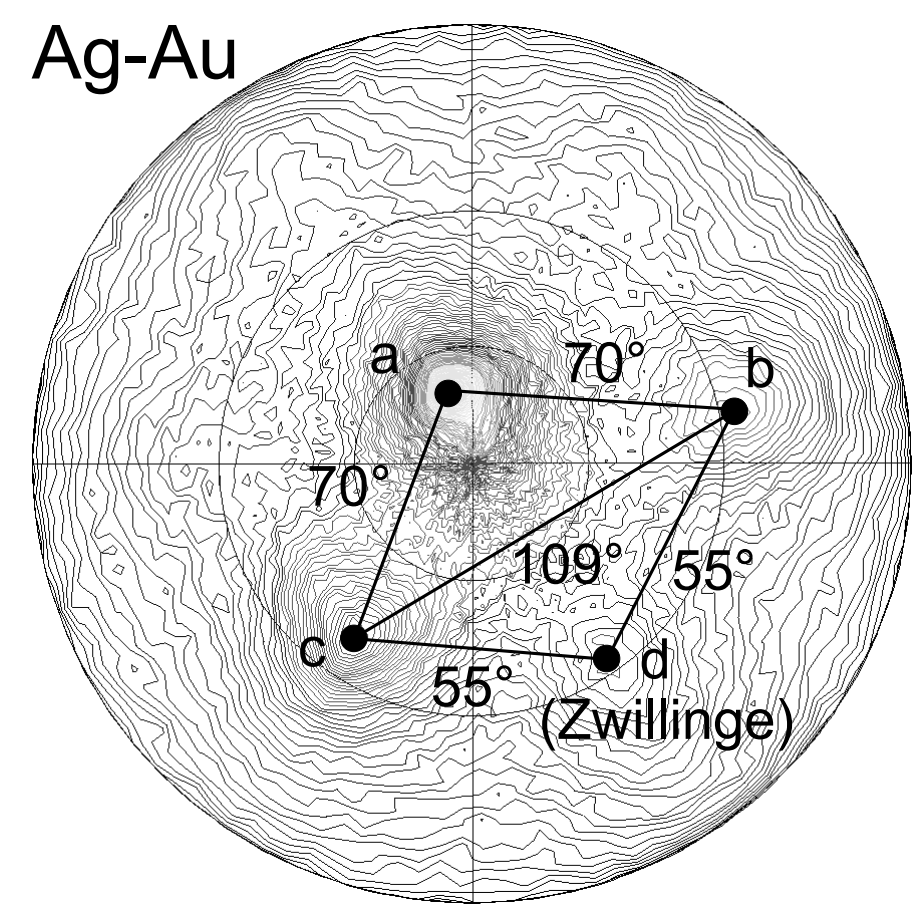

Abb. 5.1. (111) Polfigur eines Ag-Au Schichtpakets auf einem einkristallinen Silbersubstrat. Die gefundenen Reflexe sind mit den ausgefüllten Kreisen markiert. Die Winkel zwischen den verschiedenen Ebenen wurden auf den Verbindungslinien angegeben. Bei einem Substrat ohne Schichten findet man nur die Ebenenscharen $(\mathrm{a}-\mathrm{c})$.

Elektronenmikroskopische Aufnahmen belegen, daß diese in großer Zahl in die Schichten eingebaut werden. Der Einbau von Zwillingen wird durch die niedrige Stapelfehlerenergie von $20 \mathrm{~mJ} / \mathrm{m}^{2}$ bei Silber und $40 \mathrm{~mJ} / \mathrm{m}^{2}$ bei Gold begünstigt [35].

Bei dem geringen natürlichen Misfit zwischen Silber und Gold von $0.2 \%$ kann man eine große kritische Dicke erwarten. Nach Matthews findet man eine kritische Schichtdicke von 25-30 nm [68]. Ab dieser Dicke werden Misfitversetzungen von Matthews gefunden, die von der Oberfläche über $\{111\}$ Ebenen zur Grenzfläche gleiten. Da die hergestellten Schichten mit einer Einzelschichtdicke von mindestens $100 \mathrm{~nm}$ die kritische Schichtdicke überschreiten, werden eingebaute Misfitversetzungen erwartet. Aufgrund der geringen Gitterfehlpassung ist ihre Zahl jedoch gering.

\subsection{Kupfer/Gold}

Die Elemente Kupfer und Gold weisen beide eine kubisch-flächenzentrierte Struktur bei einem Gittermisfit von $12 \%$ auf. Bei den hergestellten Schichtsystemen wurde daher keine spezielle Orientierungsbeziehung zwischen den Schichten erwartet. Zur Charakterisierung der Schichten wurde eine (111) und (200) Polfigur des auf einem Kupfereinkristall aufgebrachten Schichtsystems jeweils für die Glanzwinkel des Kupfers als auch des Golds aufgenommen. Da die Kupfer 


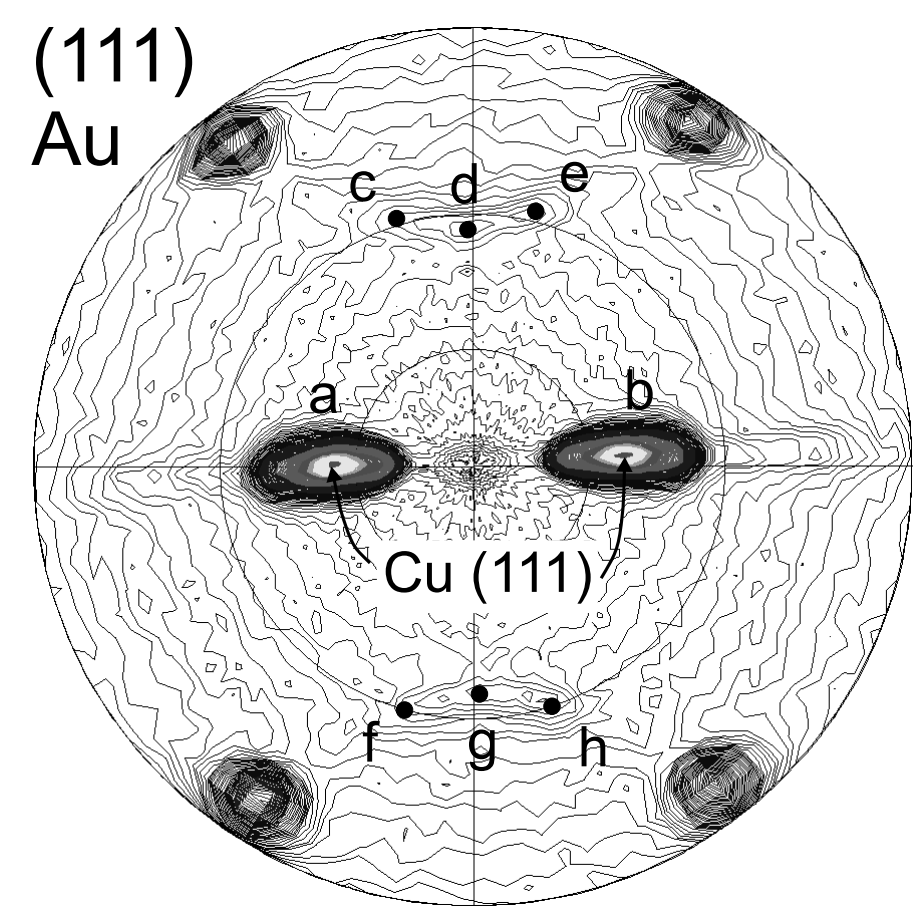

Abb. 5.2. Au (111) Polfigur eines Kupfer/Gold Schichtpakets auf einem einkristallinen Kupfersubstrat mit einer [110] Stabachsenorientierung. Die gefundenen Reflexe sind mit ausgefüllten Kreisen markiert. Die (111) Orientierung des Kupfereinkristalls wurde zusätzlich markiert.

(111) und die Gold (200) Polfigur aufgrund eines sehr ähnlichen Ebenenabstands schlecht zu trennen und die Kupfer (200) Polfigur von den starken Reflexen des einkristallinen Kupfersubstrats überlagert wird, wird in Abbildung 5.2 die Gold (111) Polfigur angegeben. In der Texturmessung findet man nur wenige verschiedene Orientierungen. Die Gold (111) Ebenen (a) und (b) befinden sich in der gleichen Orientierung wie die (111) Ebenen des Kupfereinkristalls. Rechts und links der Punkte (a) und (b) befinden sich zusätzlich noch kleinere Peaks, was die breite Form der Peaks (a) und (b) erklärt. Zusätzlich findet man unter einem Winkel von $70^{\circ}$ weitere (111) Orientierungen (c, e, f, h), die einen $55^{\circ}$ Winkel zu jeweils einer anderen (111) Orientierung neben (a) bzw. (b) aufweisen. Dies kann durch eine Zwillingsorientierung gegenüber dem darunterliegenden Kupfer erklärt werden, bei der das Gitter um 70.5 bzw. 109.5 um eine [110] Achse gedreht wird. Die weiteren Reflexe (d) und (g) ergeben sich durch die Kombination von Zwillingsorientierungen über die verschiedenen Schichten hinweg.

\subsubsection{Mikroskopische Charakterisierung der Grenzfläche}

Betrachtet man eine hochauflösende Strukturabbildung der Grenzfläche, so findet man wie durch die Texturmessung angedeutet zwei verschiedene Grenzflächentypen. Im ersten Fall gibt es die in Abbildung 5.3 dargestellte direkte epitaktische Beziehung mit einer semikohärenten Grenzfläche. Der unterschiedliche Gitterparameter wird über zusätzliche Ebenen ausgeglichen. In Abbildung 5.3 sind die zusätzlichen Ebenen an der Stelle (a) für (111) und an der Stelle (b) für (200) 

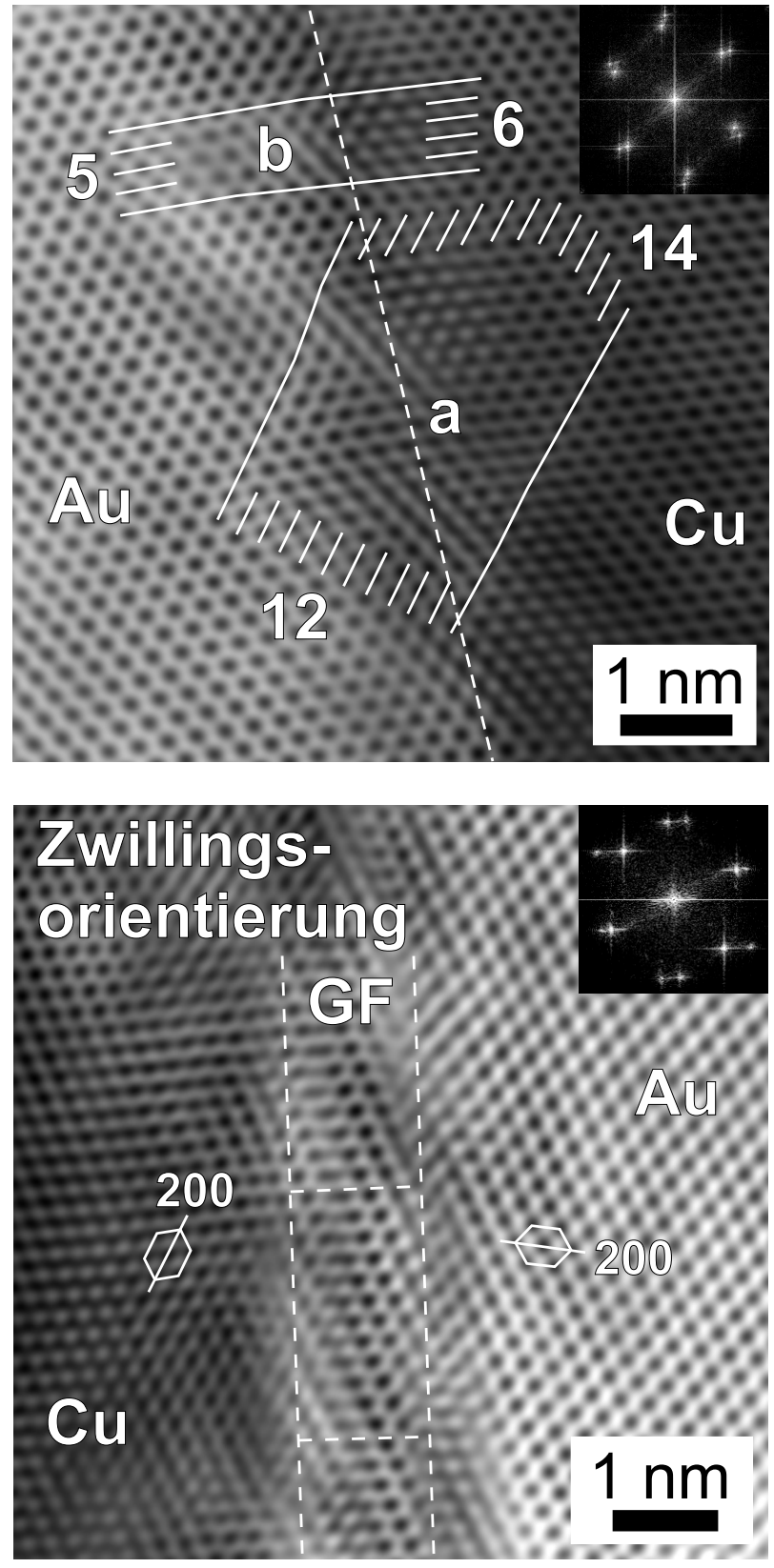

Abb. 5.3. HREM-Aufnahme einer semikohärenten Grenzfläche zwischen Kupfer und Gold. Das Gold ist auf das Kupfer aufgebracht worden. An den Stellen (a) und (b) wurde demonstriert, daß sich die Zahl der (111) und (200) Ebenen in der Grenzfläche verändert, um den unterschiedlichen Gitterparameter auszugleichen. Die Orientierung der Schichten ist zusätzlich um 2 - $3^{\circ}$ um die [110] Achse verdreht.

Abb. 5.4. HREM-Aufnahme einer semikohärenten Grenzfläche zwischen Kupfer und Gold in einer Zwillingsorientierung. Das Kupfer ist auf das Gold aufgebracht worden. An der Grenzfläche findet man eine gestörte Zone (GF) mit einer Breite von etwas über einem Nanometer, die die Zwillingsorientierung zwischen den beiden Schichten vermittelt. Zusätzlich ist eine Wiederholung der Struktur in einem Abstand von etwa $2 \mathrm{~nm}$ zu erkennen.

Ebenen exemplarisch angegeben. Die Breite der Zone, in der man das findet, beträgt ungefähr $1 \mathrm{~nm}$. Dabei wird die Orientierung zwischen den verschiedenen Gittern nicht exakt eingestellt, in HREM-Aufnahmen findet man eine Drehung von $2-3^{\circ}$ um eine [110] Achse.

Beim zweiten Grenzflächentyp entdeckt man, wie in Abbildung 5.4 dargestellt, eine Zwillingsbeziehung zwischen den Schichten. Die Grenzfläche scheint in diesem Fall in zwei Bereiche aufgeteilt zu sein. In der Zone zwischen den Gebieten (b) findet man eine gestörte Zone mit einer Breite von etwas über einem Nanometer. 


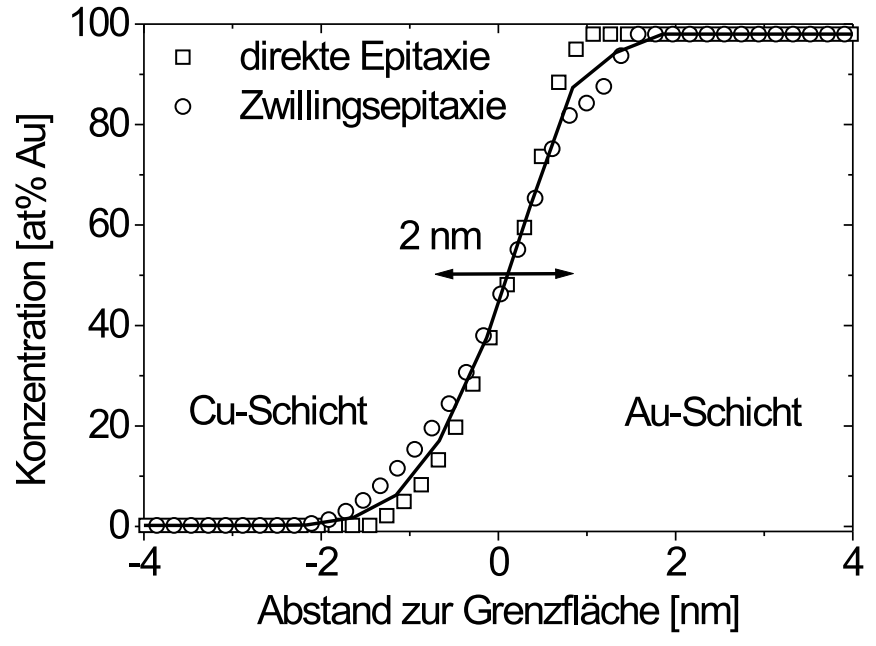

Abb. 5.5. Mit der Z-Kontrast Elektronenmikroskopie ermitteltes Konzentrationsprofil senkrecht zur Grenzfläche für die beiden Grenzflächentypen. In beiden Fällen ist eine Durchmischung der Grenzfläche auf einer Breite von $2 \mathrm{~nm}$ zu erkennen.

In der Zone sieht man eine Wiederholung des Fringemusters etwa alle $2 \mathrm{~nm}$. Die Breite der gesamten Zone, in der sich das Versetzungsnetzwerk befindet, erscheint etwas breiter als im ersten Fall. Eine chemische Analyse mit der Z-Kontrast Elektronenmikroskopie, wie sie in Abbildung 5.5 dargestellt ist, liefert jedoch in beiden Fällen eine ähnliche Durchmischungszone von $2 \mathrm{~nm}$. Die etwas breitere gestörte Zone der Zwillingsgrenzfläche ist damit nicht mit einer stärkeren Durchmischung der Grenzfläche verknüpft. Bedenkt man die Auflösungsgrenze der Methode von $1 \mathrm{~nm}$ und die zusätzlich möglichen Fehlerquellen wie z.B. eine Verkippung der Grenzfläche, so kann man eine Durchmischung außerhalb des Versetzungsnetzwerks ausschließen. Dies stimmt gut mit Messungen von Gladyszewski überein, der bei der Herstellung von Kupfer/Gold Schichten eine Durchmischungszone von 0.70-0.75 nm findet [31].

Betrachtet man einen größeren Bereich der Grenzfläche, so sind beide Grenzflächentypen vorhanden. Diese liegen teilweise sogar wie in Abbildung 5.6 dargestellt direkt nebeneinander vor. Bedenkt man, daß die Stapelfehlerenergie mit

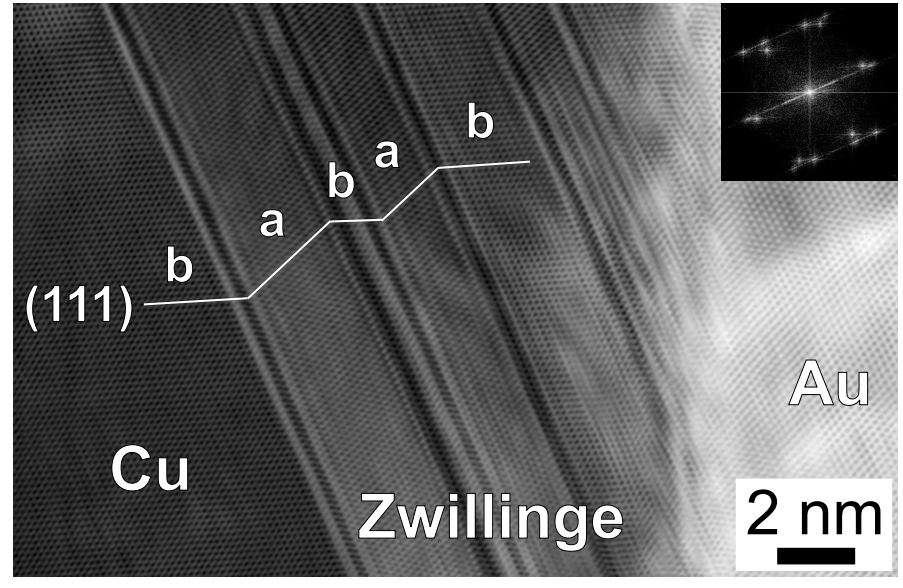

Abb. 5.6. HREM-Aufnahme einer Grenzfläche zwischen Kupfer und Gold. Die direkte Orientierungsbeziehung (a) und die Zwillingsorientierung (b) zwischen den beiden Schichten liegen direkt nebeneinander vor. 


\section{Charakterisierung der Schichtsysteme}

$40 \mathrm{~mJ} / \mathrm{m}^{2}$ im Gold und $60 \mathrm{~mJ} / \mathrm{m}^{2}$ im Kupfer [35] recht niedrig ist, so erscheint das direkte epitaktische Schichtwachstum neben einer Zwillingsorientierung verständlich.

Damit ergibt sich eine epitaktische Grenzfläche mit einer wohldefinierten Orientierungsbeziehung. Das epitaktische Schichtwachstum scheint eine generelle Eigenschaft des Kupfer-Gold-Systems zu sein. Man findet diese Textur für verschieden geschnittene Einkristalle und bei verschiedenen Herstellungsbedingungen.

\subsubsection{Epitaktisches Schichtwachstum bei großer Gitterfehlpassung}

Da der Grund für die gefundene epitaktische Schichtstruktur für das Kupfer-Gold System nicht sofort ersichtlich ist, wurden weitere Überlegungen angestellt. Da eine epitaktische Belegung der Grenzflächen im System Kupfer-Gold mit einem Energiegewinn verknüpft ist [78], würde man nach dem beim Silber-Gold System vorgestellten Modell ab einer kritischen Schichtdicke $h_{k}$ den Einbau von Misfitversetzungen zur Kompensation der elastische Spannung erwarten. Jedoch ist diese Beschreibung nach Matthews [69] bei einem Misfit von mehr als 9\% nicht mehr hilfreich. Bei einem solch großen Misfit sinkt die kritische Schichtdicke $h_{k}$ auf weniger als eine Atomlage und die Dichte der Versetzungen würde sehr hoch. Der Gleichgewichtsabstand wäre so gering, daß man nicht zwischen den Regionen guter und schlechter Passung unterscheiden könnte und die Kernbereiche der Versetzungen überlappen würden. Vergleicht man diese Grenzflächen jedoch mit einer Großwinkelkorngrenze, so findet man eine größere Ähnlichkeit. Um die Epitaxie bei Systemen mit einer großen Gitterfehlpassung zu verstehen, schlug Matthews vor [69], die Grenzfläche zwischen zwei Materialien mit großer Gitterfehlpassung ähnlichen dem Koinzidenzmodell einer Großwinkelkorngrenze [8] mit einem Koinzidenzgitter an der Grenzfläche zu beschreiben. In einem Koinzidenzgitter minimaler Energie stimmt ein möglichst großer Teil der Plätze mit dem des anderen Gitters überein und man findet eine Periodizität des Koinzidenzgitters entlang der Grenzfläche. Unterstützt wurde diese Modellvorstellung durch Rechnungen von du Plessis und van der Merwe [21] und Fletscher [25, 26]. Sie fanden steile Minima der Grenzflächenenergie falls das Verhältnis der Gitterparameter die Relation $a_{s} / a_{l}=1 / 2,2 / 3,3 / 4, \ldots, m / n$ erfüllt. Van der Merwe [115] schlug vor, daß diese steilen Minima der Grenzflächenenergie Auswirkungen auf die Bildung einer epitaktischen Grenzfläche speziell bei großen Gitterfehlpassungen haben sollten. Die Gitterkonstanten im System Kupfer-Gold erfüllen diese Relation mit 8 zu 9 sehr gut. Von Marcor wird für das System Kupfer-Gold [65], wie auch von Vook und Horng für das System Silber-Kupfer [116], das praktisch die gleiche Gitterfehlpassung wie Kupfer-Gold aufweist, auch eine direkte 


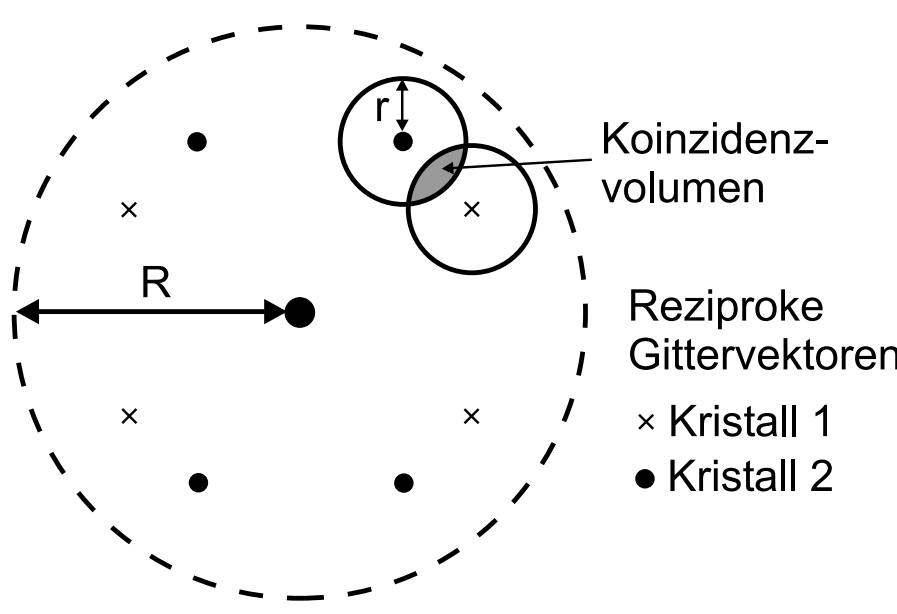

Abb. 5.7. Das Koinzidenzvolumen ergibt sich aus den überlappenden Volumina der reziproken Gittersphäre. Berücksichtigt werden alle Gitterpunkte die in einer Sphäre mit dem Radius $\mathrm{R}$ um den Ursprung im reziproken Raum liegen.

Epitaxie gefunden. In den hochaufösenden Gitterabbildungen in den Abbildungen 5.3 und 5.6 findet man Hinweise auf das erwartete Koinzidenzgitter. Im Fall der Zwillingsgrenzfläche läßt sich auch sehr schön die Periodizität der Korngrenze beobachten.

Es stellt sich nun die Frage, warum gerade diese beiden Beziehungen, die direkte und die Zwillingsorientierung, zwischen den Gittern von Kupfer und Gold eingestellt werden. Dazu betrachten Pirouz et al. [83] den allgemeinen Fall der optimalen Anpassung zwischen zwei verschiedenen Gittern. Sie gehen von der Beobachtung aus, daß eine gute Übereinstimmung der Beugungsbilder zweier Gitter mit einer guten Passung der beiden Gitter verknüpft ist. Sie ordnen den Gitterebenen der beiden Kristalle im reziproken Raum eine reziproke Gittersphäre mit dem Radius r zu. Das Koinzidenzvolumen V definiert sich aus der Summe der überlappenden Volumina der reziproken Gittersphären über niedrig indizierte Gitterebenen

$$
V=\sum_{|g|,|G|<R,|g-G|<2 r} \frac{\pi}{3}\left(4 \cdot r^{3}-3 \cdot r^{2} \cdot|g-G|+\frac{|g-G|^{3}}{4}\right)
$$

mit $\mathrm{R}$ dem Radius des berücksichtigten reziproken Raums. Dies ist in Abbildung 5.7 graphisch illustriert. Das Koinzidenzvolumen ist damit von der Position der beiden Kristalle zueinander abhängig. Man kann das Koinzidenzvolumen für ein Raster aller verschiedenen Kristallorientierungen ausrechnen und so die besten Gitteranpassungen finden. Hält man den einen Kristall fest, so bleiben noch 3 freie Parameter übrig, was eine Darstellung verkompliziert. In Abbildung 5.8 ist deshalb die Drehung um eine [110] Achse aufgetragen, wie sie aus den Texturmessungen ermittelt wurde. Man sieht, daß die direkte Gitterbeziehung und eine $70^{\circ}$ Drehung um eine [110] Achse, die einer Zwillingsorientierung entspricht, zu einem großen Koinzidenzvolumen und damit zu einer guten Gitterpassung führt. Eine Untersuchung des verbliebenen Winkelraums liefert keine größeren Koinzidenzvolumina. Man erhält so die gefundene Orientierungsbeziehung zwischen Kupfer 


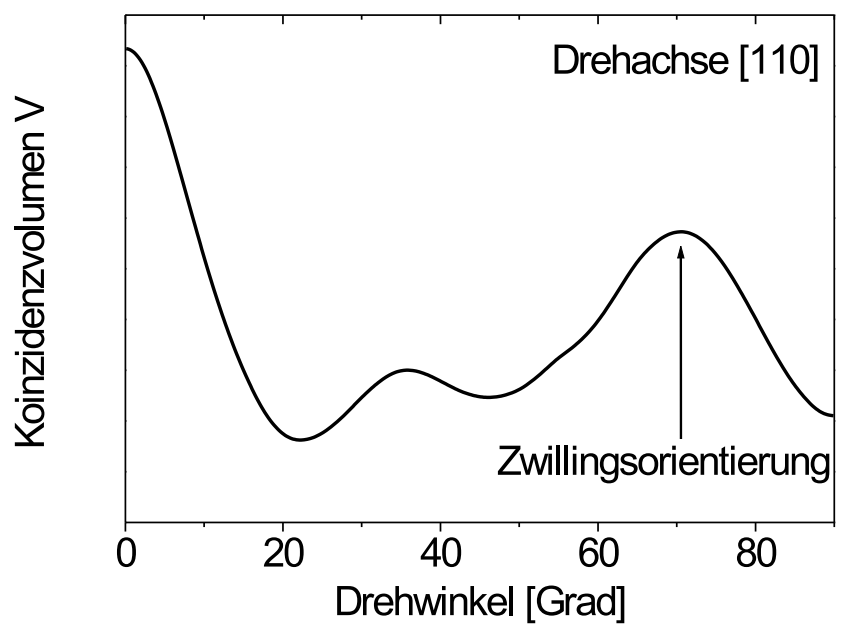

Abb. 5.8. Koinzidenzvolumen von Kupfer und Gold bei der Drehung um die [110] Achse. Als Parameter wurden $r=$ $0.2 a_{A u}^{-1}$ und $R=4 a_{A u}^{-1}$ verwendet. Neben einer direkten epitaktischen Beziehung erweist sich die Zwillingsorientierung als günstig.

und Gold aus rein geometrischen Überlegungen. Für die Zwillingsorientierung aus Abbildung 5.4 bedeutet dies, daß zwischen den Schichten ein energetisch günstiges Koinzidenzgitter existiert.

Zwischen den Überlegungen von Matthews und Pirouz besteht die Gemeinsamkeit, daß für eine epitaktische Grenzfläche eine hohe Koinzidenz der Gitterplätze gefordert wird. Eine nach dem Modell von Pirouz gefundene hohe Koinzidenz führt damit ganz allgemein zu einem kleinen Koinzidenzmisfit nach dem Modell von Matthews. Die Abweichungen an einer entsprechenden Grenzfläche werden durch Misfitversetzungen ausgeglichen. Mit dem Modell nach Pirouz können allgemein sehr leicht alle Orientierungen nach passenden Koinzidenzen abgesucht und mögliche epitaktische Grenzflächen angegeben werden. 


\section{Interdiffusion}

In diesem Kapitel werden die Ergebnisse der Interdiffusion in Silber/Gold und Kupfer/Gold Schichtsystemen vorgestellt. Das Silber-Gold System weist praktisch keine Gitterfehlpassung auf, während sich im Kupfer-Gold System die Gitterparameter um 12\% unterscheiden. Die sich daraus ergebenden Unterschiede werden im direkten Vergleich betrachtet.

\subsection{Silber/Gold}

Die Interdiffusion in Silber/Gold Schichtsystemen mit einer Einzelschichtdicke von $150 \mathrm{~nm}$ wurde bei einer Auslagerungstemperatur von $350{ }^{\circ} \mathrm{C}$ untersucht. Die Temperatur wurde so gewählt, daß die kürzeste Auslagerungszeit mehrere Minuten betrug.

\subsubsection{Experimentelle Ergebnisse}

Der Ausgangszustand wie auch die ausgelagerten Proben wurden mit dem Elektronenmikroskop charakterisiert. Man findet wie im vorherigen Kapitel dargelegt im Ausgangszustand eine epitaktische Schichtstruktur, die auch in den ausgelagerten Proben erhalten bleibt. Außerdem sind im Mittel etwa alle 150 nm Kleinwinkelkorngrenzen erkennbar, wobei dieser Abstand der Schichtdicke entspricht. Im Hellfeldbild ist keine wesentliche Veränderung der Struktur durch die Auslagerung erkennbar. Mit der Z-Kontrast Elektronenmikroskopie kann man jedoch, wie in Abbildung 6.1 gezeigt, eine deutliche Durchmischung an der Grenzfläche feststellen. Bei der dargestellten Konzentrationsanalyse wurde darauf geachtet, daß der Abstand von der nächsten Kleinwinkelkorngrenze mindestens der Eindringtiefe aus der Volumendiffusion entsprach und sich möglichst keine anderen Defekte in der Nähe befanden. Damit ist sichergestellt, daß das Konzentrationsprofil nicht durch schnelle Diffusionspfade beeinflußt wurde. In erster Näherung wurde für das Diffusionsproblem die Dickschichtlösung mit einem konzentrationsunabhängigen Diffusionskoeffizienten D angesetzt:

$$
c(x, t)=\frac{c_{0}}{2} \cdot \operatorname{erfc}\left(\frac{x}{2 \sqrt{D t}}\right)
$$




\section{Interdiffusion}

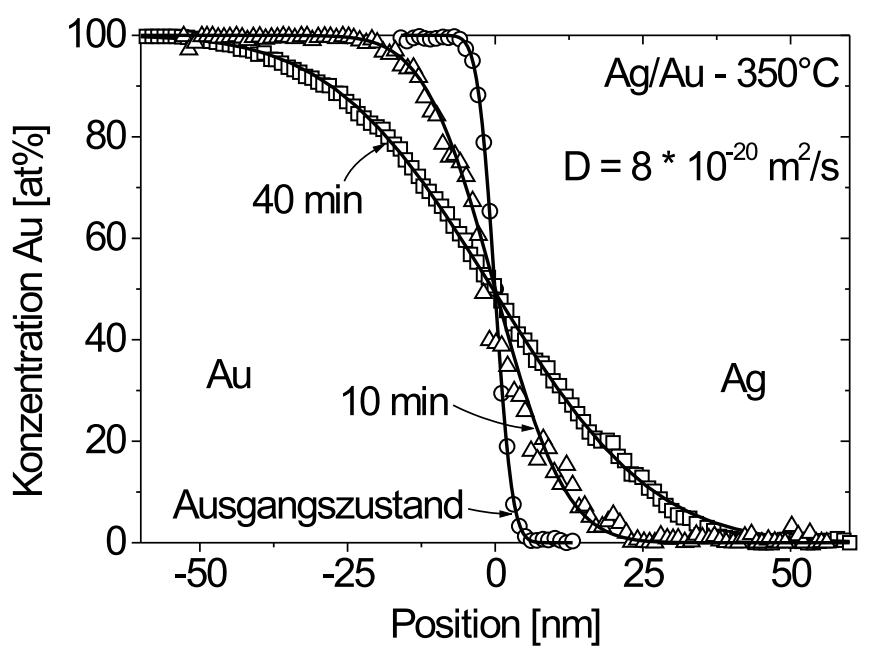

Abb. 6.1. Konzentrationsprofile an der Grenzfläche zwischen einer Silber und einer Goldschicht im Ausgangszustand und nach einer Auslagerung bei $350{ }^{\circ} \mathrm{C}$ für 10 und 40 Minuten. Die Profile wurden mit der Z-Kontrast Elektronenmikroskopie ermittelt und lagen jeweils weit von der nächsten Kleinwinkelkorngrenze entfernt. Die angegebenen Konzentrationsprofile wurden aus 6 Messungen an verschiedenen Probenstellen ermittelt. Die durchgezogene Linie ergibt sich aus einer Anpassung der Dickschichtlösung nach Gleichung 6.1.

Um die endliche Auflösung der Z-Kontrastabbildung und das Konzentrationsprofil des Ausgangszustands bei der Bestimmung des Diffusionskoeffizienten zu berücksichtigen, wurde ein Ansatz nach Hall [36] benutzt. Dort wird ein um die Anfangsbedingung modifizierter Diffusionskoeffizient D angesetzt

$$
4 D t=4 D_{m e s s} t-a^{2}
$$

mit $D_{\text {mess }}$ dem experimentell ermittelten Diffusionskoeffizienten und a dem Parameter der Funktion erfc $(x / a)$, die den unreagierten Ausgangszustand beschreibt. Damit ergibt sich für die beiden Auslagerungszustände von 10 und 40 Minuten ein Diffusionskoeffizient von $8 \cdot 10^{-20} \mathrm{~m}^{2} / \mathrm{s}$ bei $350{ }^{\circ} \mathrm{C}$.

Zusätzlich wurden SNMS Messungen an einem $\mathrm{Au} / \mathrm{Ag} / \mathrm{Au}$ Schichtpaket mit einer Einzelschichtdicke von $100 \mathrm{~nm}$ auf einem Silbereinkristall durchgeführt. Die Ergebnisse dieser Messungen sind in Abbildung $6.2 \mathrm{zu}$ sehen. Wie schon bei den Konzentrationsprofilen, die aus der Z-Kontrast Elektronenmikroskopie ermittelt wurden, wurde auch hier die Dickschichtlösung aus Gleichung 6.1 an die Profile angepaßt. Dabei ergibt sich ein Diffusionskoeffizient $\mathrm{D}=1.6 \cdot 10^{-19} \mathrm{~m}^{2} / \mathrm{s}$, der etwas über dem mit der Elektronenmikroskopie ermittelten Wert liegt. Offensichtlich wird bei den SNMS Messungen im Gegensatz zu den Messungen mit dem Elektronenmikroskop die Korngrenzdiffusion miterfaßt. 


\section{Interdiffusion}
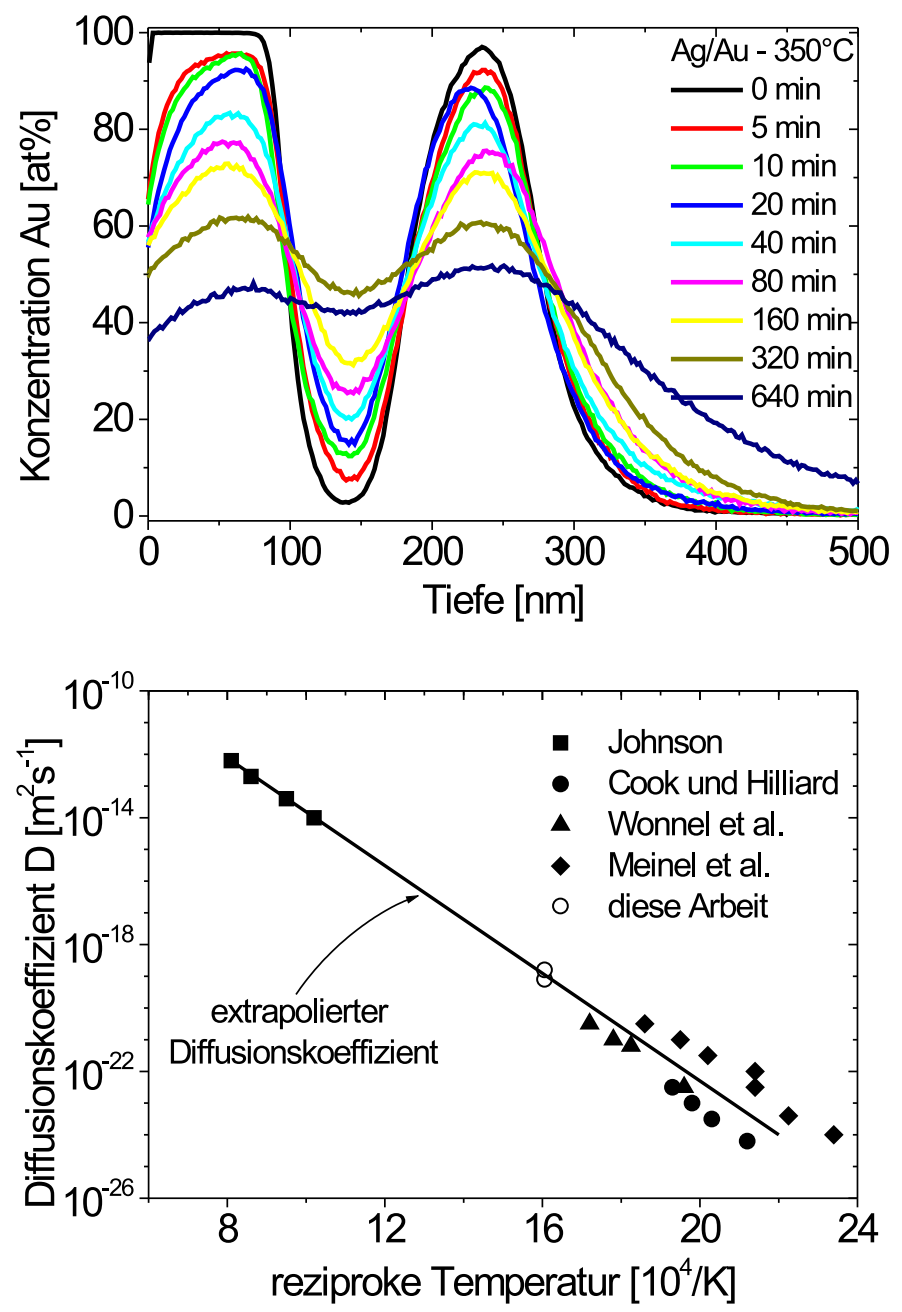

Abb. 6.2. Konzentrationsprofile einer $\mathrm{Au} / \mathrm{Ag} / \mathrm{Au}$ Schichtprobe mit einer nominellen Einzelschichtdicke von $100 \mathrm{~nm}$ auf einem Silbereinkristall, die bei $350{ }^{\circ} \mathrm{C}$ für verschieden lange Zeiten ausgelagert wurde. Die Profile wurden mit der SNMS ermittelt.

\begin{abstract}
Abb. 6.3. Diffusionsdaten des Silber-Gold Systems aus der Literatur $[14,45,70,121]$ für eine $\mathrm{Ag}_{50} \mathrm{Au}_{50}$ Legierung und die Ergebnisse der vorliegenden Arbeit. Die durchgezogene Linie ergibt sich durch Extrapolation der Hochtemperaturdaten von Johnson.
\end{abstract}

Die ermittelten Diffusionsdaten sind in Abbildung 6.3 zusammen mit Daten aus der Literatur aufgetragen. Die Ergebnisse stimmen mit den Literaturwerten gut überein. Es gibt keinen Hinweis, daß Spannungen, die aufgrund unterschiedlicher Diffusionsgeschwindigkeit auftreten könnten, die Interdiffusion in Schichten der Dicke von 100 bis $150 \mathrm{~nm}$ mit untersuchten Diffusionswegen von einigen Nanometern beeinflussen. Auch findet man unter diesen Bedingungen bei den untersuchten Proben keinen Hinweis auf den Einfluß von Grenzflächenbarrieren. Eine Hemmung der Diffusion durch eine kurzreichweitige Ordnung, wie man sie in der Literatur für wenige Nanometer dicke Schichten findet [123], ist unter diesen Umständen auch nicht zu erkennen.

Betrachtet man das SNMS-Konzentrationsprofil aus Abbildung 6.2 entfernt von den Grenzflächen an den Schichtmitten, so ist dort ein sofortiges Absinken der Konzentrationswerte erkennbar. Auch fällt die durch Volumendiffusion nicht erklärbare Anreicherung von Silber an der Oberfläche des Schichtpakets auf. Es 


\section{Interdiffusion}

scheint, als ob die Korngrenzdiffusion einen wichtigen Einfluß auf den Diffusionsverlauf ausübt. Um dies zu beschreiben, werden im folgenden entsprechende Lösungen des Diffusionsproblems für Volumen- und Korngrenzdiffusion angegeben, die auf die Randbedingungen eines Schichtpakets angepaßt wurden. Anhand dieser Lösungen werden die bisher nur qualitativen Überlegungen über den Diffusionsmechanismus überprüft.

\subsubsection{Volumendiffusion in einem Schichtpaket}

Da das Verständnis der beteiligten Prozesse im Vordergrund steht, wird im folgenden der Einfachheit halber mit konzentrationsunabhängigen Diffusionskoeffizienten gearbeitet. Im Fall der Volumendiffusion kann man aus der Quellenlösung

$$
c(x, t)=\frac{s}{\sqrt{\pi D t}} \cdot \exp \left(-\frac{x^{2}}{4 D t}\right)
$$

mit s der Menge der eindiffundierenden Substanz den Konzentrationsverlauf bei Eindiffusion einer Schicht von $x_{1}$ bis $x_{2}$ mit der Anfangskonzentration $c_{0}$ in eine unendlich ausgedehnte Matrix durch eine Integration beschreiben:

$$
\begin{aligned}
c(x, t) & =\int_{x_{1}}^{x_{2}} \frac{c^{\prime}}{\sqrt{\pi D t}} \cdot \exp \left(-\frac{\left(x^{\prime}-x\right)^{2}}{4 D t}\right) d x^{\prime} \\
& =\frac{c_{0}}{2}\left[\operatorname{erf}\left(\frac{x_{2}-x}{2 \sqrt{D t}}\right)-\operatorname{erf}\left(\frac{x_{1}-x}{2 \sqrt{D t}}\right)\right]
\end{aligned}
$$

wobei sich c' aus den Anfangsbedingungen errechnet. Ein ganzes Schichtpaket ergibt sich aus der Summe der Einzelschichten nach Gleichung 6.4. In dem untersuchten Schichtpaket befinden sich die Schichten jedoch nicht in einer unendlich ausgedehnten Matrix, sondern haben eine freie Oberfläche, über die hinweg keine Diffusion erfolgen kann. Um dies zu berücksichtigen, wird die Methode der Spiegelung nach Stefan $[47,101]$ benutzt. Man setzt dazu neben dem Ausgangsprofil ein an der Oberfläche gespiegeltes Konzentrationsprofil an und berechnet die Zeitentwicklung nach dem Fickschen Gesetz. Da die neue Konzentrationsverteilung symmetrisch um die Oberfläche ist, kompensieren sich die Ströme durch die Oberfläche und man hat keinen Materietransport durch die Oberfläche. Für ein Schichtsystem $\mathrm{Au} / \mathrm{Ag} / \mathrm{Au}$ auf einem Silbereinkristall ergibt sich damit aus der Volumendiffusion die folgende Zeitentwicklung: 


\section{Interdiffusion}

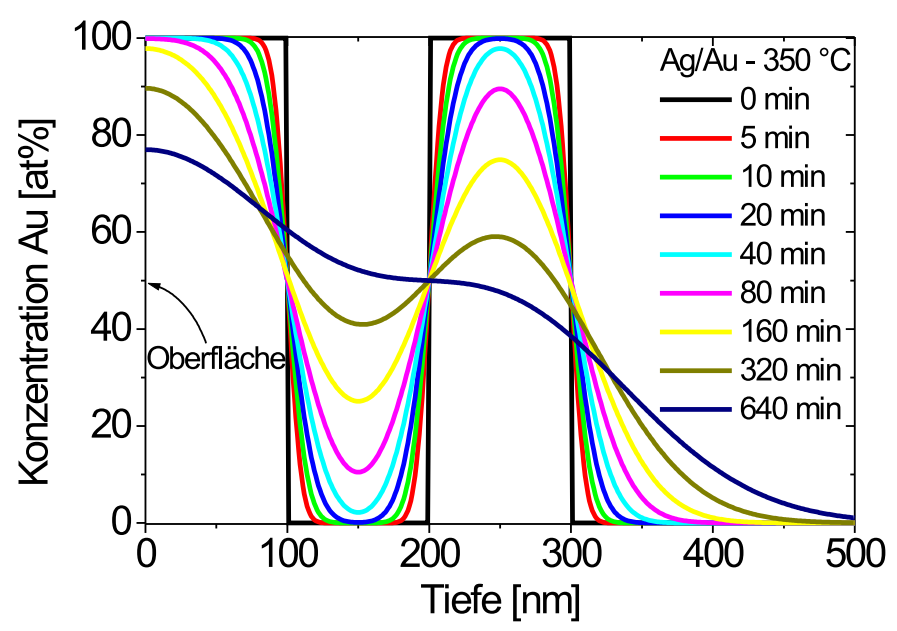

Abb. 6.4. Konzentrationsprofile eines $\mathrm{Au} / \mathrm{Ag} / \mathrm{Au}$ Schichtpakets für die verschiedenen Auslagerungszeiten der SNMS Messung, wie sie sich aus Gleichung 6.5 ergeben. Der verwendete Volumendiffusionskoeffizient betrug $\mathrm{D}=8 \cdot 10^{-20} \mathrm{~m}^{2} / \mathrm{s}$.

$$
\begin{array}{r}
c(x, t)=\frac{1}{2} \quad\left[\operatorname{erf}\left(\frac{-2 h-x}{2 \sqrt{D t}}\right)-\operatorname{erf}\left(\frac{-3 h-x}{2 \sqrt{D t}}\right)+\operatorname{erf}\left(\frac{h-x}{2 \sqrt{D t}}\right)-\right. \\
\left.\operatorname{erf}\left(\frac{-h-x}{2 \sqrt{D t}}\right)+\operatorname{erf}\left(\frac{3 h-x}{2 \sqrt{D t}}\right)-\operatorname{erf}\left(\frac{2 h-x}{2 \sqrt{D t}}\right)\right]
\end{array}
$$

mit $\mathrm{h}=100 \mathrm{~nm}$ der Einzelschichtdicke. Für einen Volumendiffusionskoeffizienten $\mathrm{D}=8 \cdot 10^{-20} \mathrm{~m}^{2} / \mathrm{s}$ ergeben sich für die gleichen Zeiten wie in der SNMS Messung die in Abbildung 6.4 angegebenen Konzentrationsprofile. Es fällt die starke Abweichung des Konzentrationsprofils im Vergleich zu den Messungen in Abbildung 6.2 in der Nähe der Oberfläche auf. Die Volumendiffusion führt zu einem Konzentrationsmaximum an der Oberfläche wie es jedoch nicht in den Messungen beobachtet wird.

Aus dieser Lösung kann man die Konzentrationsänderungen in den Silber- und den Goldschichten wie sie sich aus einer Volumendiffusion ergeben würden berechnen. Diese sind zusammen mit den Meßwerten aus den SNMS Messungen in Abbildung 6.5 dargestellt. Bei den Rechnungen wurde der Volumendiffusionskoeffizient $\mathrm{D}=8 \cdot 10^{-20} \mathrm{~m}^{2} / \mathrm{s}$ verwendet, wie er sich aus der elektronenmikroskopischen Untersuchung für reine Volumendiffusion ergab. Man findet, wie in Abbildung 6.5 dargestellt, im Gegensatz zu den Meßwerten eine Konzentrationsänderung erst zu späteren Zeiten. Zusätzlich unterscheiden sich die experimentell ermittelten Peakkonzentrationen der ersten und der zweiten Goldschicht kaum. Der Unterschied bei der Lösung nach 6.5 ist jedoch sehr groß, wie es der Vergleich der Peakkonzentrationen mit der maximalen Konzentration an der Oberfläche in Abbildung 6.5 zeigt. Der quantitative Verlauf der Konzentration aus Abbildung 6.2 über die erste Schicht unterscheidet sich signifikant, da die Lösung nach 6.5 die maximale Konzentration in der ersten Schicht immer an der Oberfläche erreicht. 


\section{Interdiffusion}

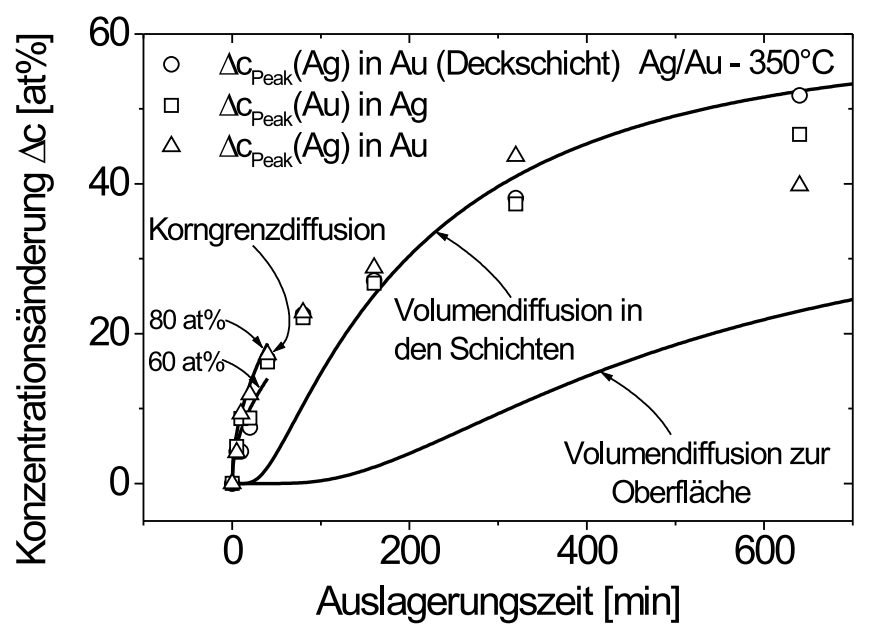

Abb. 6.5. Konzentrationsänderung in der Mitte der Silberund Goldschichten aus den SNMS-Messungen gegenüber den unreagierten Schichten. Die durchgezogenen Linien ergeben sich aus angepaßten Modellen zur Volumen- und Korngrenzdiffusion (Gl. 6.5 und 6.9 mit den Korngrenzkonzentrationen $\quad c_{b}^{\text {konst }}=$ $60 / 80$ at\%).

\subsubsection{Einfluß der Korngrenzdiffusion}

Zu der Volumendiffusion ist der Transport über die Korngrenzen zu berücksichtigen. Man erkennt eine Bergaufdiffusion in der Nähe der Oberfläche gegen den Silbergradienten. Bezieht man die Korngrenzen mit in die Betrachtung ein, so erhält man die in der schematischen Darstellung 6.6 angegebenen Diffusionsströme. Über die Korngrenze kann Silber durch die Goldschicht an die freie Oberfläche gelangen und von dort ins Volumen diffundieren. Eine laterale Integration liefert dann erst die mit der SNMS gemessene mittlere Silberkonzentration. Damit erfolgt die Diffusion ganz normal in Richtung des Konzentrationsgradienten. Da sich das Konzentrationsprofil der ersten und der zweiten Schicht nicht wesentlich unterscheidet, kann man davon ausgehen, daß der Transport über die Korngrenze und die Oberfläche das Material schneller anliefert, als es wieder ins Volumen diffundieren kann. An der Oberfläche stellt sich eine stationäre Konzentration ein.

Die Korngrenzdiffusion soll nun beschrieben und an die im Experiment herrschenden Randbedingungen angepaßt werden. Eine allgemeine Darstellung dieser Problematik findet man zum Beispiel bei Kaur und Gust [49]. Für die Diffusion in der Korngrenze mit der Breite $\delta$ setzt man die Gültigkeit der Fickschen Gesetze mit einem Korngrenzdiffusionskoeffizienten $D_{b}$ an. Für die folgenden Betrachtungen soll die Korngrenze entlang des Ursprungs der z-Achse liegen. Wie bei Kaur und

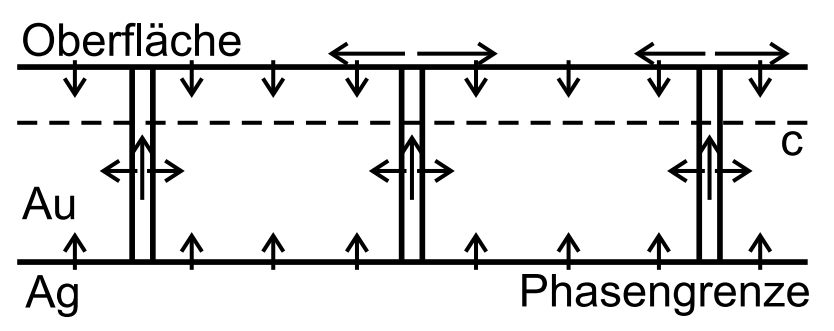

Abb. 6.6. Darstellung der verschiedenen Diffusionsströme in der obersten Goldschicht. Eine SNMS Messung würde eine gemitttelte Konzentration entlang der gestrichelten Linie ergeben. 


\section{Interdiffusion}

Gust [49] beschrieben, ergibt sich für die zeitliche Entwicklung der Konzentration an der Korngrenze die Differentialgleichung

$$
\frac{\partial c_{g}}{\partial t}=D_{b} \frac{\partial^{2} c_{g}}{\partial z^{2}}+\left.\frac{2 D}{\delta} \frac{\partial c_{g}}{\partial y}\right|_{|y|=\delta / 2}
$$

mit $c_{g}$ der Konzentration an der Korngrenze. Der erste Term auf der rechten Seite repräsentiert die Diffusion entlang der Korngrenze und der zweite Term beschreibt die Diffusion aus der Korngrenze in das Korn. Im allgemeinen Fall muß noch über einen Segregationsfaktor eine mögliche Anreicherung in der Korngrenze berücksichtigt werden, der hier jedoch vernachlässigt wird. Um diese Gleichung zu lösen, wird ein Ansatz wie bei Fisher [24] verwendet. Dabei wird davon ausgegangen, daß die Diffusion in das Korn hauptsächlich über die Korngrenze stattfindet und daß sich in der Korngrenze schnell ein stationärer Zustand einstellt. Unter diesen Bedingungen erhält man eine Differentialgleichung zweiter Ordnung, die das Konzentrationsprofil in der Korngrenze beschreibt:

$$
\frac{\partial^{2} c_{b}(z, t)}{\partial z^{2}}=\frac{2 \sqrt{D}}{\sqrt{\pi t} \delta D_{b}} c_{b}(z, t)
$$

Für eine Korngrenze in einem halbunendlichen Medium ergibt sich daraus eine exponentiell abfallende Konzentrationsverteilung

$$
c_{b}=c_{0} \cdot \exp \left(-\frac{\sqrt{2} \sqrt[4]{D} z}{\sqrt[4]{\pi t} \sqrt{\delta D_{b}}}\right)
$$

Das Konzentrationsprofil außerhalb der Korngrenze wird durch die Lösung des Diffusionsproblems einer konstanten Quelle der Konzentration $c_{b}$ in einem halbunendlichen Medium beschrieben. Im vorliegenden Fall ist der Konzentrationsverlauf in einem Abstand von $50 \mathrm{~nm}$ von der Phasengrenze interessant, der der Mitte der Schicht und damit der Position der maximalen Konzentration in den ausgemessenen Silber/Gold Schichtpaketen entspricht. Zur Abschätzung der Konzentration in der Korngrenze wird die gemessene Konzentration an der Oberfläche herangezogen. Sie beträgt nach der SNMS Messung zwischen 30 und 40 at\% Silber. Die Konzentration in der Korngrenze muß damit an der Oberfläche im Abstand von $100 \mathrm{~nm}$ von der Silberschicht mindestens 40 at\% Silber betragen. Mit der Auslagerungszeit von 5 Minuten ergibt sich nach Gleichung 6.8 bei einer $0.5 \mathrm{~nm}$ breiten Korngrenze mindestens ein Korngrenzdiffusionskoeffizient $D_{b}$ von $5 \cdot 10^{-16} \mathrm{~m}^{2} / \mathrm{s}$. Dieser Wert ist sehr viel kleiner als der Diffusionskoeffizient einer Großwinkelkorngrenze in Silber/Gold, den Soria und Sacedón [99] bei $350{ }^{\circ} \mathrm{C}$ mit $4.5 \cdot 10^{-11} \mathrm{~m}^{2} / \mathrm{s}$ angeben. Die große Differenz bestätigt den mit dem Elektronenmikroskop gewonnenen Eindruck, daß es sich um Kleinwinkelkorngrenzen 


\section{Interdiffusion}

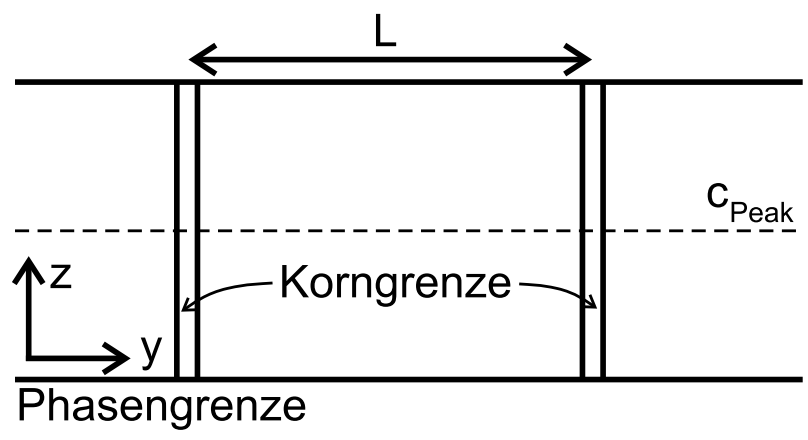

Abb. 6.7. Schematische Darstellung einer Schicht, in der die Korngrenzen einen mittleren Abstand L haben. Die maximale mittlere Konzentration in einer Schicht wird in der Mitte an der gestrichelten Linie auftreten. Die SNMS ermittelt eine durchschnittliche Konzentration entlang dieser Linie.

handelt. Mit diesem Wert ergibt sich nach Gleichung 6.8 in einem Abstand von $50 \mathrm{~nm}$ von der Phasengrenze eine Korngrenzkonzentration $c_{b}$ von 65 at\% Silber. Die reale Korngrenze wird jedoch von beiden Seiten gespeist, so daß ein schnellerer Anstieg der Konzentration in der Korngrenze erwartet werden kann. Dem wirkt das Absinken der Konzentration durch die Volumendiffusion an der Grenzfläche entgegen. In erster Näherung wird deshalb eine konstante Konzentration $c_{b}(z)$ zwischen 60 und 80 at\% Silber in der Mitte der Schichten angesetzt. Die in den Schichten vorliegende Geometrie ist schematisch in Abbildung 6.7 dargestellt. Damit ergibt sich die mittlere Konzentration im Zentrum einer Schicht mit einem mittleren Abstand der Korngrenzen L wie folgt:

$$
\begin{aligned}
\bar{c}(z, L, t) & =\frac{c_{b}(z)}{L} \int_{-L / 2}^{L / 2} \operatorname{erfc}\left(\frac{x}{2 \sqrt{D t}}\right) d x \\
& \approx \frac{c_{b}^{k o n s t}}{L} \int_{-L / 2}^{L / 2} \operatorname{erfc}\left(\frac{x}{2 \sqrt{D t}}\right) d x
\end{aligned}
$$

Die Näherung mit einer konstanten Konzentration ist gerechtfertigt, da schon für den Ansatz nach Fisher ein stationäres Profil vorausgesetzt wird. Der Ansatz eines stationären Profils wird auch durch experimentelle Ergebnisse gestützt [49]. Als freie Parameter bleiben dann der Volumendiffusionskoeffizient D, die Konzentration in den Korngrenzen $c_{b}^{k o n s t}$ und der Abstand der Korngrenzen L. Mit dem schon bestimmten Volumendiffusionskoeffizient $\mathrm{D}=8 \cdot 10^{-20} \mathrm{~m}^{2} / \mathrm{s}$ und einer Konzentration $c_{b}^{\text {konst }}$ zwischen 60 und 80 at\% ergibt sich für einen Korngrenzabstand von $150 \mathrm{~nm}$ eine gute Anpassung an die in Abbildung 6.5 dargestellten Daten. Dieser Abstand stimmt recht gut mit den aus den TEM-Bildern gewonnenen Daten überein, bei denen der Abstand der Kleinwinkelkorngrenzen auch $150 \mathrm{~nm}$ entsprach. Die Anpassung kann natürlich nur die Anfangssteigung wiedergeben, da mit steigender Auslagerungszeit die Volumendiffusion einen erheblichen Anteil an der Konzentrationsänderung gewinnt. 


\section{Interdiffusion}

Die Interdiffusion in den untersuchten Silber/Gold Schichtsystemen läßt sich damit komplett mit einer Überlagerung von Korngrenz-, Oberflächen- und Volumendiffusion beschreiben. Es gibt auch keinen Hinweis auf die Behinderung der Diffusion durch die Einstellung einer Nahordnung. Dies mag damit zusammenhängen, daß der Einfluß der Nahordnung bei der Untersuchung von Yang [123] mit einer deutlich kleineren Diffusionsweite im Bereich unter einem Nanometer und den damit viel steileren Konzentrationsgradienten aufgetreten ist. In diesem Bereich mußten zudem Gradientenenergien bei der Analyse der Diffusion berücksichtigt werden. Damit erscheint es plausibel, daß diese kleinen Störungen die Diffusion viel stärker beeinflussen als im Fall größerer Diffusionsweiten, wie sie in dieser Untersuchung vorlagen und damit hier nicht zu beobachten sind.

\subsection{Kupfer/Gold}

Für die Untersuchung der Interdiffusion ohne den Einfluß von Ordnungsphasen wurde eine Auslagerungstemperatur von $430{ }^{\circ} \mathrm{C}, 20^{\circ} \mathrm{C}$ über der höchsten Ordnungstemperatur der $\mathrm{CuAu}(\mathrm{II})$ Phase von $410{ }^{\circ} \mathrm{C}$, gewählt. Die Diffusionszeiten für die Untersuchung der Frühstadien sind sehr kurz, so daß eine Differenz der realen Auslagerungszeiten aufgrund der Ankoppelung der Proben an das Salzbad für die verschiedenen Proben zu erwarten ist. Generell ergeben sich für die elektronenmikroskopischen Proben aufgrund der direkten Ankoppelung die kleinsten Abweichungen in den Auslagerungszeiten. Bei den Auslagerungszeiten der anderen Proben wurde eine entsprechende Korrektur durchgeführt.

\subsubsection{Experimentelle Ergebnisse}

Für eine makroskopische Charakterisierung wurde ein Tiefenprofil mit der SNMS für verschiedene Auslagerungszeiten ermittelt. Um Abweichungen aufgrund von Variationen der Herstellungsparameter zwischen verschiedenen Schichtpaketen zu vermeiden, wurden alle Messungen an einer Probe durchgeführt. Das Ergebnis der Messung findet sich in Abbildung 6.8. Bereits nach der ersten Auslagerung von 2 Sekunden ist ein Interdiffusionsprofil an den Grenzflächen zu sehen. Auch findet man in den Goldschichten 4 at\% Kupfer. Dieser Zustand bleibt zunächst stabil. Erst bei der für 13 Sekunden ausgelagerten Schichtprobe findet man eine Fortsetzung der Interdiffusion. In der Mitte der Schichten ist an einigen Stellen ein Plateau mit konstanter Konzentration vorhanden. Das Absinken der Konzentration an der Oberfläche ist, wie schon bei den Silber-Gold-Schichten beobachtet, durch den Transport von Kupfer über Korngrenzen an die Oberfläche zu erklären. Auf der Oberfläche verteilt sich das Kupfer sehr schnell und dringt durch normale Volumendiffusion wieder in das Material ein. 


\section{Interdiffusion}
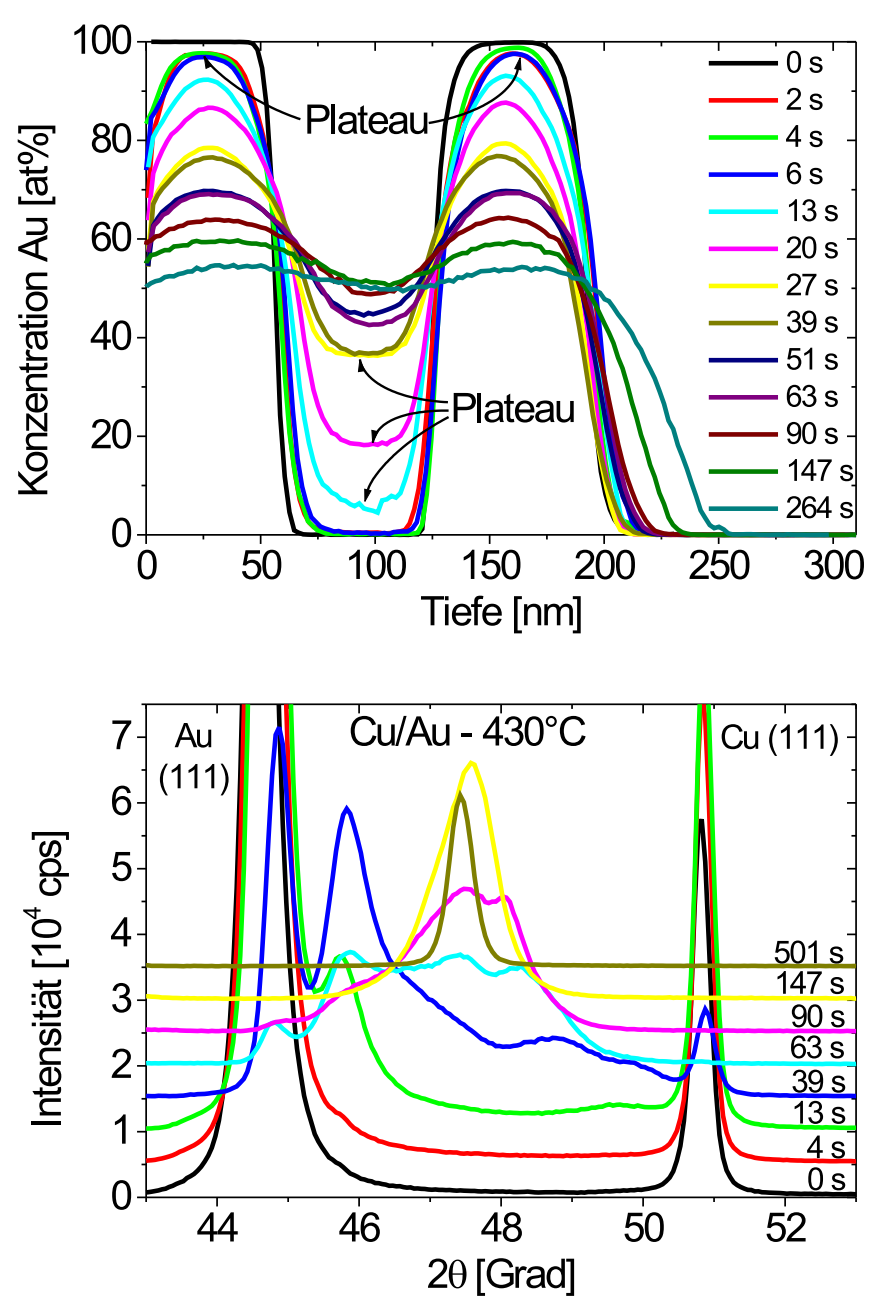

Abb. 6.8. SNMS-Messung eines für verschiedene Zeiten bei $430 \quad{ }^{\circ} \mathrm{C}$ ausgelagerten $\mathrm{Au} / \mathrm{Cu} / \mathrm{Au}$ Schichtpakets auf einem Kupfereinkristall. Nach einer anfänglichen Interdiffusion stoppt die Reaktion. Erst bei der für 13 Sekunden ausgelagerten Schichtprobe findet man eine Fortsetzung der Reaktion.
Abb. 6.9. Röntgendiffrakto- gramm einer bei $430{ }^{\circ} \mathrm{C}$ für verschiedene Zeiten ausgelager- ten Kupfer/Gold-Schichtprobe mit einer Einzelschichtdicke von $60 \mathrm{~nm}$. Nach einer Zeit von 13 Sekunden treten erste neue Reflexe im Spektrum auf. Die Reaktion endet mit einer kompletten Durchmischung der beiden Phasen.

Zusätzlich wurden die gleichen Auslagerungszustände wie bei der SNMS-Messung mit Hilfe der Röntgendiffraktometrie untersucht. Auch hier wurden alle Messungen an einer einzigen Probe durchgeführt. In Abbildung 6.9 wurde der Ausschnitt des Röntgenspektrums, in dem die $\{111\}$-Reflexe von Kupfer, Gold und entsprechenden Legierungen liegen sollten, aufgetragen. In den ersten Sekunden der Interdiffusion ist eine leichte Erhöhung der Intensität des Diffraktogramms und eine Verschiebung des Goldpeaks zu beobachten. Dies ist mit einer Interdiffusion bzw. entstehenden Spannungen an der Grenzfläche zu erklären. Aufgrund der Ergebnisse der SNMS Messung ist eine Durchmischung an der Grenzfläche und eine Anreicherung der Goldschicht mit Kupfer die plausibelste Erklärung. Nach einem Verharren in diesem Zustand findet man nach 13 Sekunden neue Reflexe im Spektrum, was mit der Bildung einer kontinuierlichen Mischungszone wie sie für die Interdiffusion zu erwarten wäre nicht verständlich ist. Dieser Prozeß wird bis zur Etablierung der endgültigen Legierung fortgesetzt. Neben den erlaubten Reflexen der kubisch-flächenzentrierten Struktur wurden keine Überstrukturreflexe gefunden. 


\section{Interdiffusion}

Aufgrund der SNMS und der Röntgendiffraktometriedaten ist also festzustellen, daß im Fall von Kupfer/Gold die Frühstadien der Interdiffusion durch eine zweistufige Reaktion gekennzeichnet sind. Dieses Verhalten ist aufgrund makroskopischer Diffusionsmodelle nicht verständlich. Die Mechanismen der beiden Reaktionsstadien sollen im folgenden durch mikroskopische Untersuchungen aufgeklärt werden.

\subsubsection{Frühstadium der Interdiffusion}

Das Frühstadium der Interdiffusion bei $430{ }^{\circ} \mathrm{C}$ im Kupfer-Gold System ist, wie in Abbildung 6.10 dargestellt, durch eine $15 \mathrm{~nm}$ breite Defektzone gekennzeichnet. Die Defektzone findet sich schon nach der kürzesten untersuchten Auslagerungszeit von 2 Sekunden und bleibt in ihrer Ausdehnung und Form bei weiterer Auslagerung stabil. Da dieser Zustand nicht dem Gleichgewichtszustand entspricht, handelt es sich um einen metastabilen Zustand. Die Defekte enden auf der Seite des Kupfers an einer einheitlichen Position, während ihre Ausdehnung in das Gebiet des Golds stärker variiert. Die Defekte verlaufen unter einem flachen Winkel zur Grenzfläche, in der gezeigten Aufnahme sind es $23^{\circ}$. Dies ist mit einer $\{111\}$ Habitusebene verträglich. Im Fall der Zwillingsgrenze liegt der Defekt auf der gemeinsamen $\{111\}$ Ebene des Kupfers und des Golds, im anderen Fall der direkten

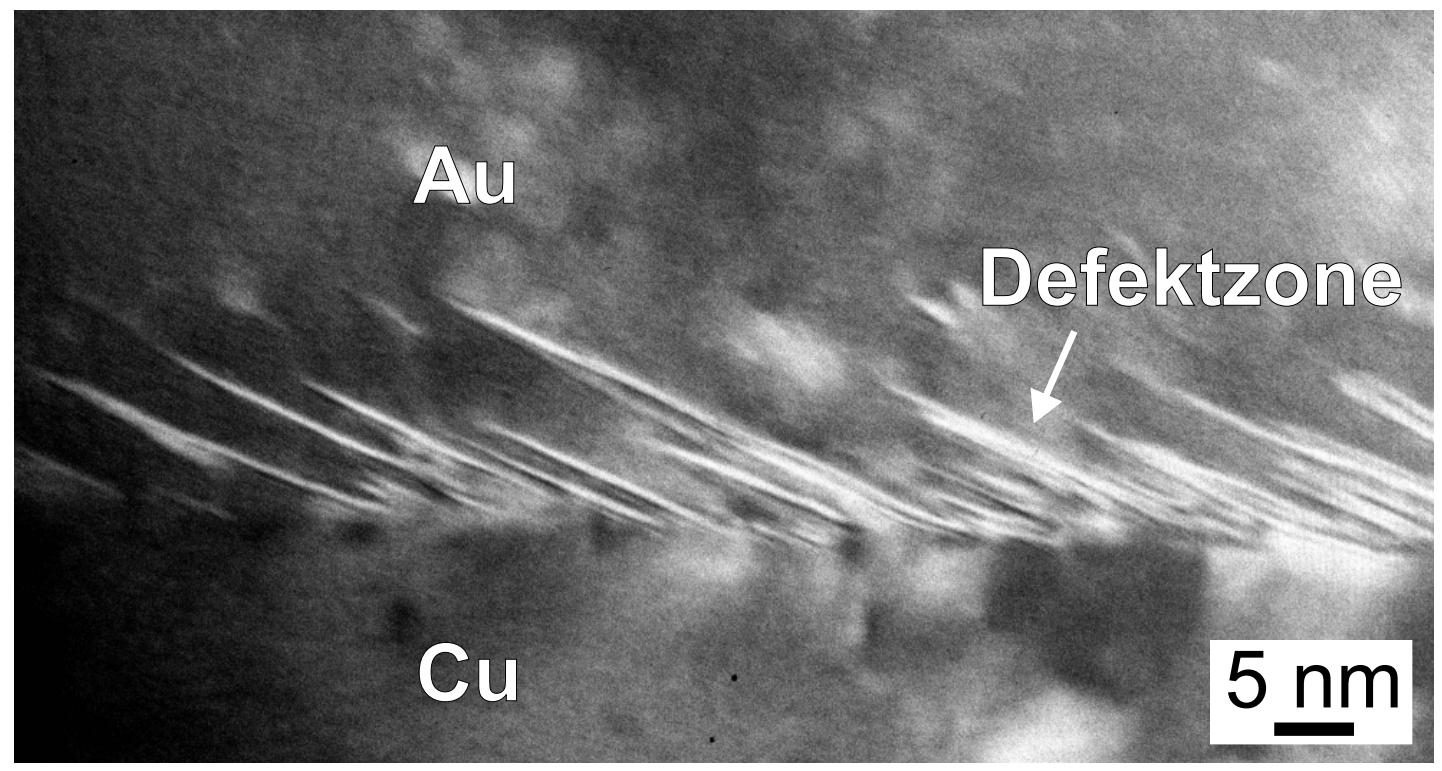

Abb. 6.10. Hellfeldaufnahme einer für 5 Sekunden bei $430{ }^{\circ} \mathrm{C}$ ausgelagerten Grenzfläche zwischen einer Kupfer- und einer Goldschicht. Eine Defektzone ist entlang der ursprünglichen Grenzfläche zu sehen. 


\section{Interdiffusion}

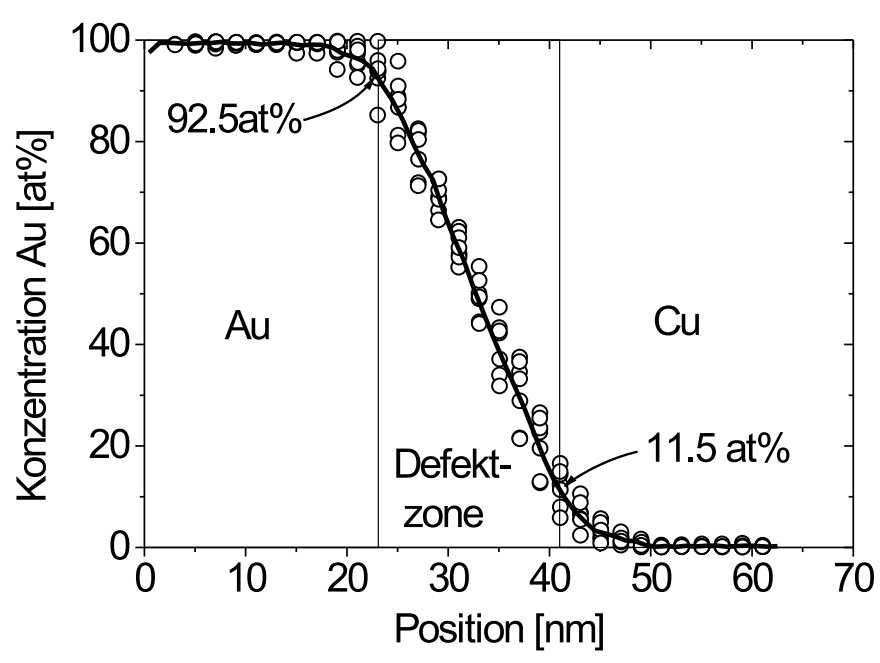

Abb. 6.11. Chemische Analyse der Grenzflächenregion mit der ZKontrastelektronenmikroskopie einer bei $430{ }^{\circ} \mathrm{C}$ für 5 Sekunden ausgelagerten Kupfer/Gold Schichtprobe. Die mittlere Grenze der Defektzone ist durch die senkrechten Striche markiert.

Epitaxie wird eine der $\{111\}$ Ebene ausgewählt. Mit der Z-Kontrast Elektronenmikroskopie wurde eine chemische Analyse der Defektregion durchgeführt. Dabei ist die Einstellung einer Zonenachse, in der die Defekte in den Hellfeldaufnahmen sichtbar sind, nicht sinnvoll, da dann eine Unterscheidung zwischen den dynamischen Bildanteilen und dem chemischen Kontrast nicht möglich ist. Die Probe wurde deshalb um $3^{\circ}$ aus der [110] Zonenachsenorientierung herausgekippt, wobei sich nun die Defekte in der Projektion überlagern und die Auswertung nur eine mittlere Konzentration ergibt. Man findet ein stabiles Konzentrationsprofil für verschiedene Positionen an der Grenzfläche wie es in Abbildung 6.11 angegeben ist. Zusätzlich wurden die Grenzen der Defektzone ermittelt. Die Grenzen der Defektzone variieren in den ausgewerteten Fällen um weniger als $1 \mathrm{~nm}$ von der in Abbildung 6.11 angegebenen mittleren Position. Damit zeigt sich, daß die Defektstruktur eng mit dem Konzentrationsprofil verknüpft ist. Die Randkonzentrationen, bei denen keine Defekte eingebaut werden, sind mit etwa 10 at\% auf beiden Seiten recht gering. Jedoch kann die Analyse aufgrund der Festlegung von Eichpunkten vorgegebener Konzentration in gewissem Abstand von der Defektzone keine Aussage darüber treffen, ob in der Kupfer- oder Goldschicht bereits eine erhöhte Konzentration der jeweils anderen Komponente vorliegt.

\subsubsection{Charakterisierung der Defektstruktur}

Zum weiteren Verständnis dieses metastabilen Zustands wird zunächst die Struktur der Defektzone untersucht. Betrachtet man eine hochauflösende Strukturabbildung der Grenzflächenregion wie sie in Abbildung 6.12 für eine reagierte Zwillingsgrenzfläche dargestellt ist so findet man für die Defekte eine plattenförmige

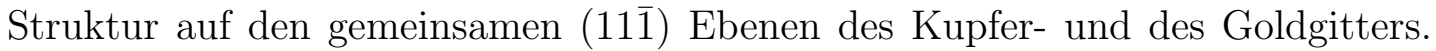
Die weiße Linie entlang der Defektstruktur trennt das Kupfer- und das Goldgitter voneinander. Die Defekte beginnen sehr schmal im Gold und verbreitern sich 


\section{Interdiffusion}

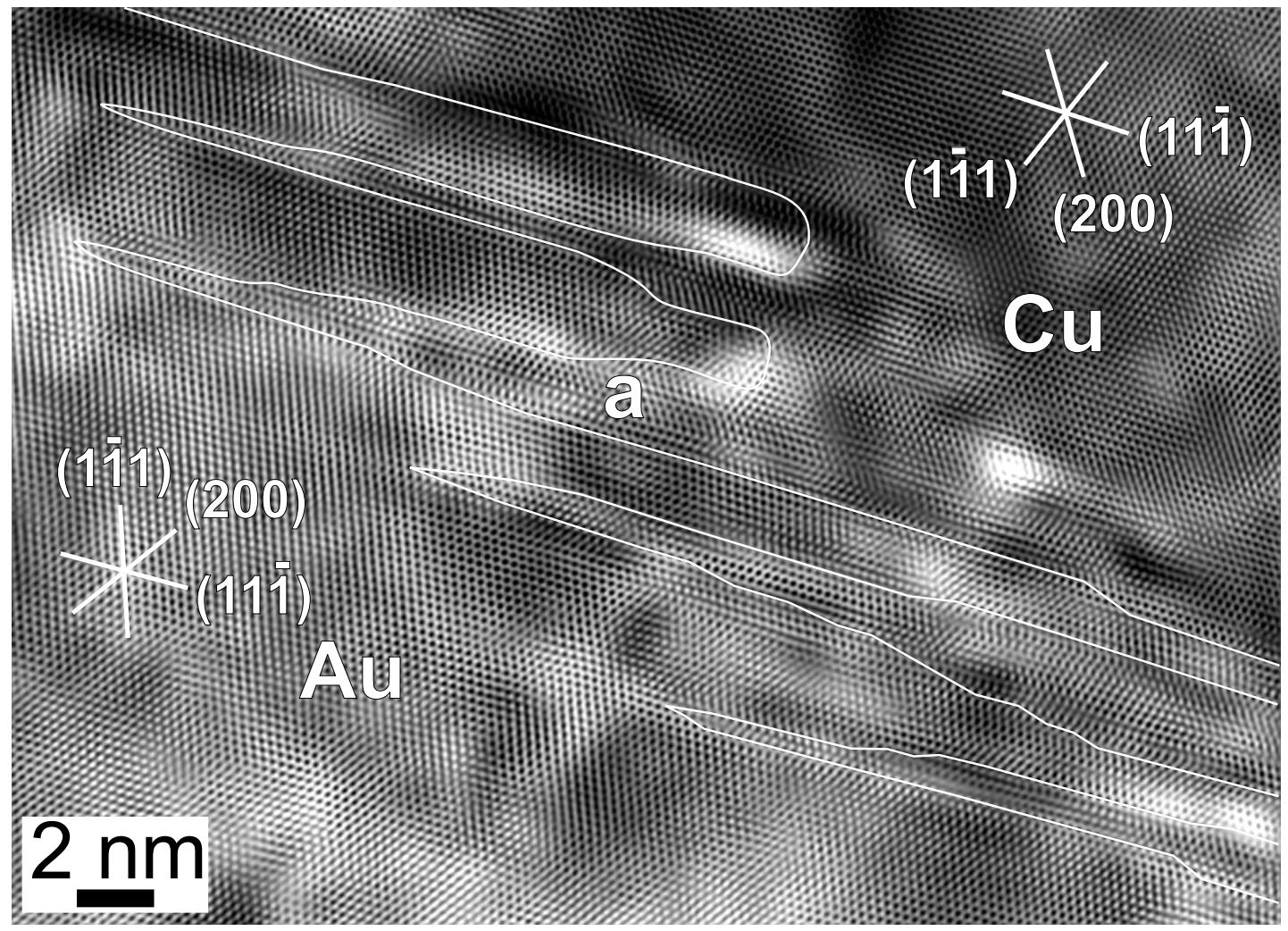

Abb. 6.12. HREM-Aufnahme einer Zwillingsgrenzfläche zwischen Kupfer und Gold, die für 5 Sekunden bei $430{ }^{\circ} \mathrm{C}$ ausgelagert wurde. Zur Unterdrückung des Rauschanteils wurde die Aufnahme fouriergefiltert. Die Defektzone besteht aus plattenförmigen Defekten auf den gemeinsamen (11̄i) Ebenen des Kupfer- und des Goldgitters.

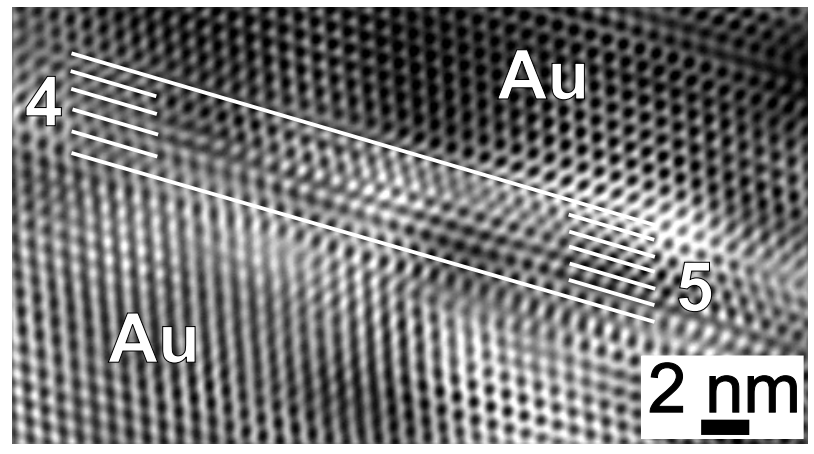

Abb. 6.13. HREM-Aufnahme der Spitze der gefundenen Defektstruktur in der Goldschicht. Man findet eine eingeschobene (111) Gitterebene.

dann. Um die plattenförmige Defektstruktur auf den gemeinsamen (11̄̄) Ebenen der beiden Gitter genauer zu verstehen, wird zunächst die Spitze der Defekte im Gold betrachtet. Wie in Abbildung 6.13 gezeigt, ist dort eine zusätzlich eingeschobene Ebene zu sehen. Außerdem erkennt man in der Defektzone Verschiebungen von Gitterebenen, wie sie durch Shockley-Partialversetzungen erzeugt werden. 


\section{Interdiffusion}

Shockley-Partialversetzungen können durch Aufspaltung einer vollständigen Versetzung nach folgendem Schema gebildet werden:

$$
\vec{b}=\frac{a}{2}[101] \Rightarrow \frac{a}{6}[112]+\frac{a}{6}[2 \overline{1} 1]=\vec{b}_{P 1}+\vec{b}_{P 2}
$$

Dabei ist a die Gitterkonstante, $\vec{b}$ der Burgersvektor der vollständigen Versetzung und $\vec{b}_{P 1}$ und $\vec{b}_{P 2}$ die Burgersvektoren der Partialversetzungen.

Zur Identifikation der Struktur an der Spitze der Defekte wurden verschiedene Defekttypen modelliert. Als Ausgangspunkt wurde ein Defekt an einer Zwillingsgrenzfläche, ähnlich der Anordnung in Abbildung 6.12, verwendet und verschiedene Stapelfolgen der $\{111\}$ Ebenen mit jeweils einer eingeschobenen Ebene betrachtet. Beim Typ 1 wird nur die zusätzliche Gitterebene in ein perfektes Goldgitter, ähnlich einem durch Kondensation von Zwischengitteratomen entstandenen extrinsischen Versetzungsring, eingebaut. Der Typ 2 besteht aus einer ausgedehnten Zone mit hexagonaler Stapelfolge. Die Defekttypen 1 und 2 führen zu keiner Abgleitung des Goldgitters entlang des Defekts. Beim Defekttyp 3 und 4 handelt es sich um einen 4 beziehungsweise 5 Ebenen Zwilling. Sie führen zu einer Abgleitung entlang des Defekts. Die verschiedenen Typen sind in Abbildung 6.14 zusammen mit den sie erzeugenden Strukturelementen graphisch dargestellt. Zur Bildsimulation der angegebenen Strukturen wurde das Programmpaket EMS32 von Stadelmann [100] eingesetzt. Eine Relaxation der geometrischen Atompositionen wurde nicht durchgeführt, da es sich immer noch um eine dichtest gepackte Struktur handelt und die Simulation nur der Bestimmung der Stapelfolge, nicht jedoch der Feinstruktur der interatomaren Abstände dient. Da die chemische Zusammensetzung des Defekts unbekannt ist, wurde der Defekt nur aus Goldatomen aufgebaut. Exemplarisch werden in Abbildung 6.15 die Simulationsergebnisse für eine Probendicke von $23 \mathrm{~nm}$ dargestellt. Dies entspricht ungefähr der Probendicke der im folgenden angegebenen Probenstellen. Die optischen Parameter entsprechen denen des Philips CM 200 FEG wie sie in Tabelle 3.3 aufgeführt sind. Alle HREM-Aufnahmen wurden im Rahmen der experimentellen Genauigkeit im Scherzerfokus aufgenommen. Deshalb wurden für die Bildsimulation die Defokuswerte um den Scherzerfokus, der bei dem verwendeten Gerät bei $-43.3 \mathrm{~nm}$ liegt, variiert.

Vergleicht man die Simulationsergebnisse mit einer typischen Probenstelle an der Spitze des Defekts, wie sie in Abbildung 6.16 dargestellt ist, so ergibt sich die beste Übereinstimmung mit dem Defekttyp 1. Die Defekttypen 3 und 4 liefern ein zu breites Defektbild. Der Defekttyp 2 besitzt in der Ebene oberhalb bzw. unterhalb der eingeschobenen Ebene keine gute Übereinstimmung mit dem experimentellen Bild. Es wird also an der Spitze des Defekts zunächst eine zusätzliche Ebene in das 


\section{Interdiffusion}
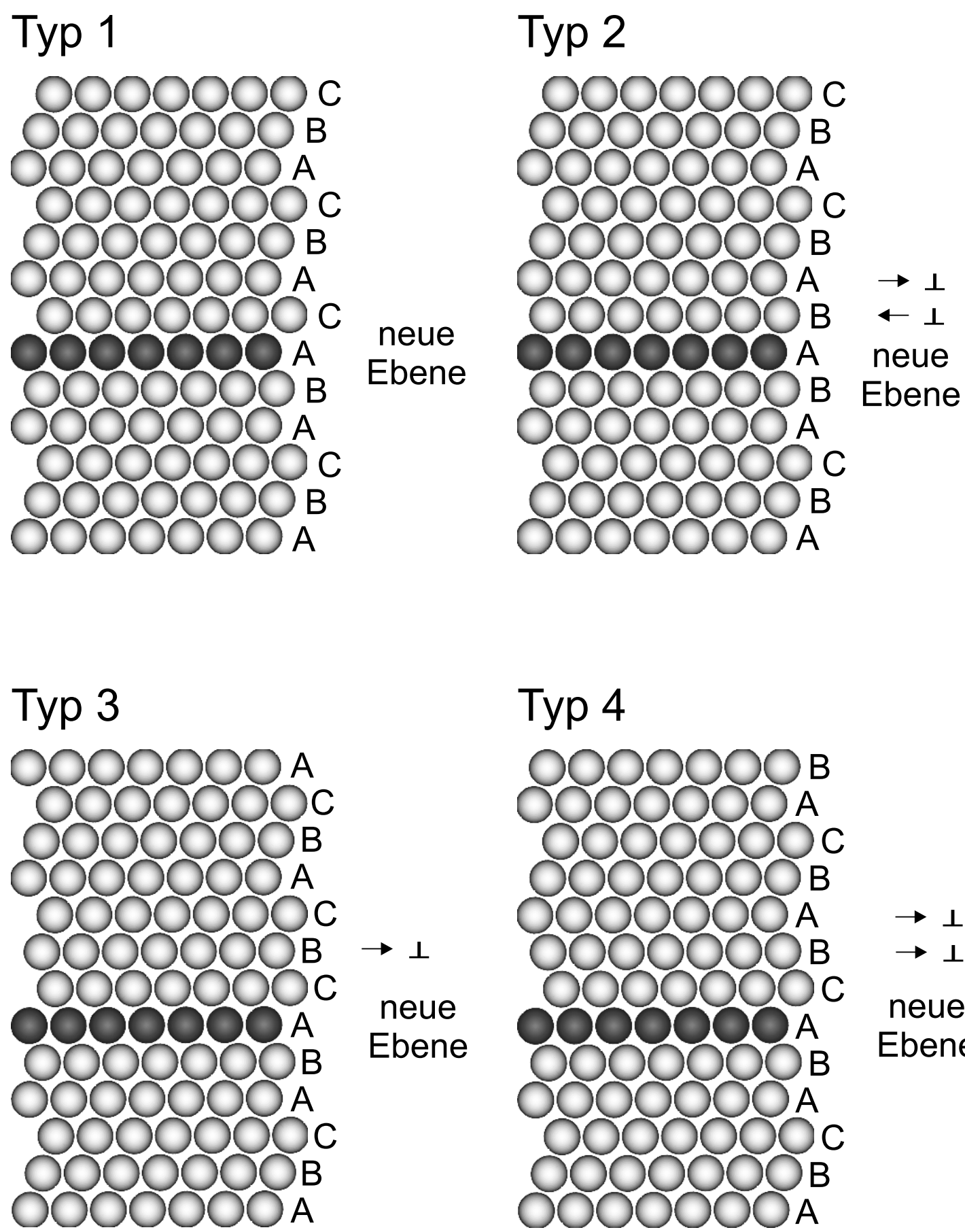

Typ 4

0000000 B 0000000 A 0000000 c 0000000 B 000000 A $\rightarrow \perp$ 0000000 B $\rightarrow \perp$ 000000C neue 0000000 A B Ebene OOPOOOOA $0000000 \mathrm{C}$ 0000000 B 0000000 a

Abb. 6.14. Strukturelle Darstellung der verschiedenen Defekttypen. Typ 1: extrinsischer Stapelfehler, Typ 2: hexgonale Stapelfolge, Typ 3: 4 Ebenen Zwilling, Typ 4: 5 Ebenen Zwilling. Die jeweils veränderten Strukturelemente sind rechts neben der Struktur angegeben. Bei den Versetzungen handelt es sich um Shockley-Partialversetzungen. 


\section{Interdiffusion}

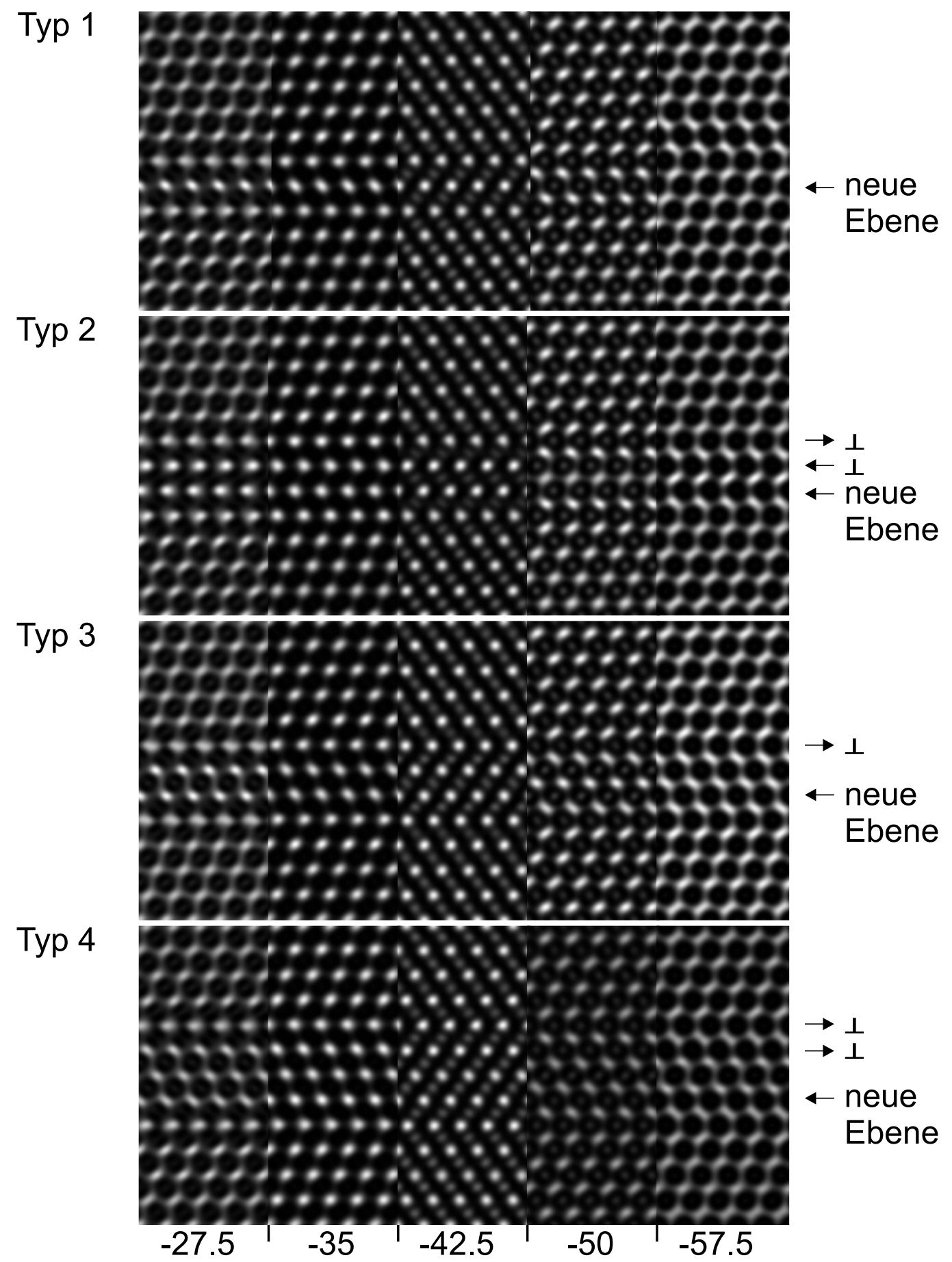

Abb. 6.15. Simulationen der verschiedenen Defekttypen 1 bis 4 für verschiedene Defokuswerte $-27.5,-35,-42.5,-50$ und $-57.5 \mathrm{~nm}$. Die teilweise sichtbaren „half period“Fringes werden in den Rechnungen überbetont und sind im experimentellen Bild aufgrund von Dämpfungseffekten nicht sichtbar. 


\section{Interdiffusion}

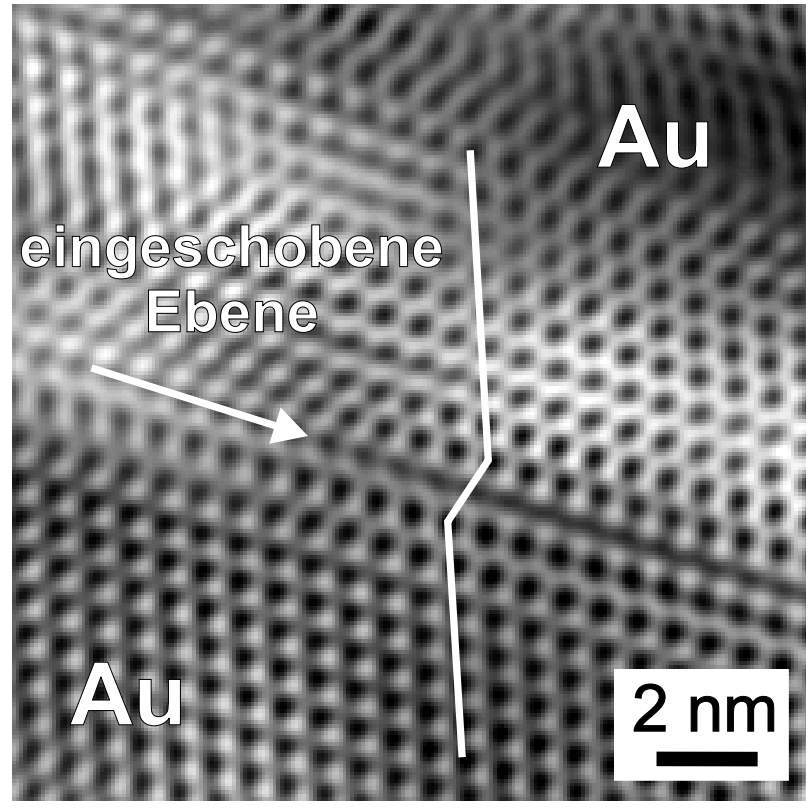

Abb. 6.16. HREM-Aufnahme der Defektzone an der Spitze eines Defekts. Die Probe wurde bei $430{ }^{\circ} \mathrm{C}$ für 5 Sekunden ausgelagert. Die weiße Linie folgt einer (111) Ebene über den Defekt hinweg. Durch Vergleich mit den elektronenmikroskopischen Simulationen kann der Defekt als eingeschobene Ebene (Typ 1 in Abbildung 6.15) identifiziert werden.

Goldgitter eingefügt. Eine solche eingeschobene Ebene entspricht einem extrinsischen Stapelfehler, der von Frank-Partialversetzungen umrandet wird. Die Ebenen werden daher an ihrer Spitze mit einer Frank-Partialversetzungen mit einem Burgersvektor von $b_{P}^{\text {Frank }}=\frac{a}{3}[11 \overline{1}]$ abgeschlossen. Die Frank-Partialversetzung selbst ist seßhaft und kann nicht gleiten. Bei Materialien mit einer hohen Stapelfehlerenergie kann sich unter Energiegewinn eine Shockley-Partialversetzung auf der eingeschobenen Ebene bilden

$$
\frac{a}{3}[11 \overline{1}]+\frac{a}{6}[112] \Rightarrow \frac{a}{2}[110] \quad,
$$

wie sie für Materialien mit hoher Stapelfehlerenergie energetisch günstig ist, wird hier jedoch nicht beobachtet.

Bewegt man sich in Richtung der Grenzfläche an diesem Defekt entlang, so findet man eine Konstellation wie in Abbildung 6.17 dargestellt. Der Defekt ist durch das Laufen von 3 Shockley-Partialversetzungen auf aufeinanderfolgenden Ebenen, ähnlich den Defekttypen 3 und 4, verbreitert worden. Es zeigt sich, daß die Struktur der ursprünglichen Grenzfläche einen entscheidenden Einfluß auf die Verbreiterung des Defekts hat. Damit ergeben sich Shockley-Partialversetzung mit den Burgersvektoren $b_{P 1}^{\text {Shockley }}=\frac{a}{6}[112]$ und $b_{P 2}^{\text {Shockley }}=\frac{a}{6}[2 \overline{1} 1]$, die auf den (11̄i) Ebenen gleitfähig sind. Befindet sich die Grenzfläche wie in diesem Fall in einer Zwillingsorientierung, so wird die Verbreiterung des Defekts durch Gleiten von Shockley-Partialversetzungen ähnlich den Defekttypen 3 und 4 realisiert. Dadurch wird der Kern des Defekts in eine Zwillingsorientierung gebracht. Ab einer gewissen Dicke des Defekts werden weitere Gitterebenen eingeschoben. Im Falle des Defekts (a) aus Abbildung 6.12 findet man zum Beispiel 3 zusätzlich eingeschobene Ebenen. An der Grenzfläche zum Kupfer geht dieser Defektbereich dann 


\section{Interdiffusion}

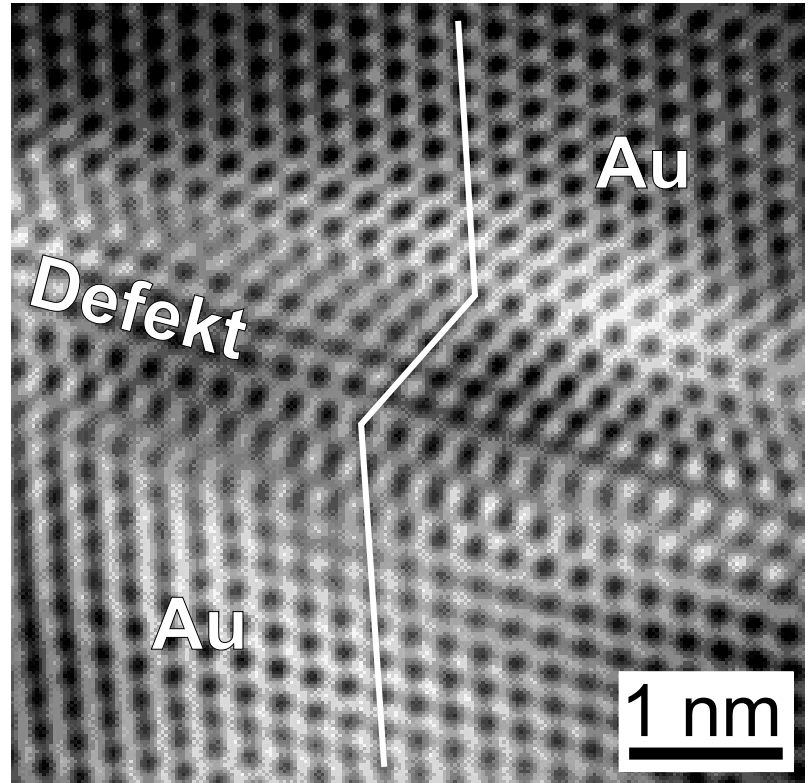

Abb. 6.17. HREM-Aufnahme einer verbreiterten Defektzone zwischen einer Kupfer- und einer Goldschicht nach einer Wärmebehandlung von 5 Sekunden bei $430^{\circ} \mathrm{C}$. Die weiße Linie folgt einer (111) Ebene über den Defekt hinweg. Der Defekt wurde durch gleiten von ShockleyPartialversetzungen verbreitert.

epitaktisch in das Kupfergitter über. Zusammengefaßt wird die Defektstruktur in der Abbildung 6.18 dargestellt. Die gefundenen Shockley-Partialversetzungen gleiten auf der gemeinsamen (11) Ebene des Kupfer und des Goldgitters. Die Frank-Partialversetzung dagegen kann sich nur durch Klettern in der gleichen (111) fortbewegen. Liegt an der Grenzfläche eine direkte epitaktische Beziehung vor, so ergibt sich ein etwas anderes Verhalten wie bei der Zwillingsgrenzfläche. An der Spitze der Defekte befindet sich auch hier eine eingeschobene Ebene die von einer Frank-Partialversetzung abgeschlossen wird. Zur weiteren plastischen Verformung tragen jedoch weitere vollständige Versetzungen bei. In diesem Fall ist es nicht notwendig, daß Shockley-Partialversetzungen streng auf aufeinander folgenden Ebenen gleiten müssen, um so den Defektbereich zu erweitern. Es ist jedoch auch hier möglich, daß auch die Versetzungen unter Energiegewinn in Shockley-Partialversetzung aufspalten. Durch die gleichmäßige Verteilung der

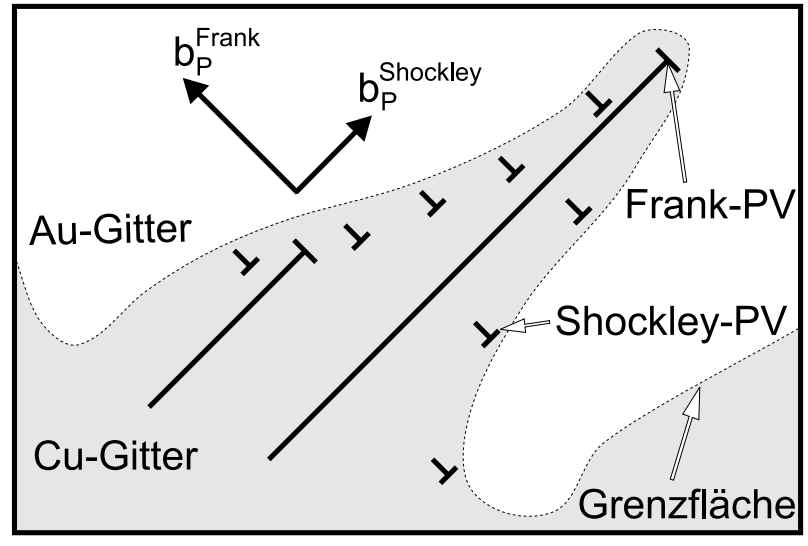

Abb. 6.18. Schematische Darstellung der Versetzungsstruktur in einer ausgelagerten Kupfer/Gold Zwillingsgrenzfläche. Die FrankPartialversetzungen sind über die Diffusionszone verteilt. An der Grenzfläche zwischen den Gittern von Kupfer und Gold wird von den Shockley-Partialversetzungen die Zwillingsorientierung entsprechend eingestellt. 


\section{Interdiffusion}

Versetzungen über die Diffusionszone ist an der direkt epitaktischen Grenzflächen keine ausgeprägte Defektstruktur erkennbar. Im Vorgriff sei dazu auf die Abbildung 6.28 verwiesen. Auf der linken Seite ist die ausgeprägte Defektstruktur an einer Zwillingsgrenzfläche zu sehen, während im Fall der direkten epitaktischen Grenzfläche keine ausgezeichnete Struktur auszumachen ist.

\subsubsection{Modellbildung für die Frühstadien der Diffusion}

Im folgenden soll eine Beschreibung des Diffusionsproblems, das zum Stopp der Interdiffusion im Reaktionsschritt I führt, ausgearbeitet werden. Zu Beginn des Reaktionsschritts I steht ein Materialtransport zwischen Schichten. Durch die Gitterfehlpassung werden Spannungen in der Diffusionszone erzeugt und eine unterschiedliche Beweglichkeit der einzelnen Komponenten führt zu einem Leerstellenungleichgewicht. Dies erzeugt eine Kraft auf die in der epitaktischen Grenzfläche vorhandenen Defekte und treibt sie in die Diffusionszone. Um sich dieser komplexen Situation zu nähern, wird zunächst nur die Diffusion unter dem Einfluß von elastischen Spannungen betrachtet. Die Behandlung der elastischen Spannungen in der Näherung eines isotropen Festkörpers folgt den Modellen von Larchè und Cahn $[58,59]$. Dort wird das Diffusionspotential M um Spannungsterme erweitert. Im folgenden wird mit der Einsteinkonvention gearbeitet, d.h. über gleiche Indizes wird summiert. Ausgangspunkt ist das 1. Ficksche Gesetz

$$
J=-B \cdot \frac{\partial M(c)}{\partial x}
$$

mit $\mathrm{M}(\mathrm{c})$ dem Diffusionspotential und B der Beweglichkeit. Für eine substitutionelle Legierung ergibt sich das Diffusionspotential und die Beweglichkeit wie folgt:

$$
\begin{aligned}
M(c) & =\frac{\partial G_{m}}{\partial c}=\mu_{A}(c)-\mu_{B}(c) \\
B & =\frac{D c(1-c)}{R T}
\end{aligned}
$$

mit D einem geeignet gewählten Diffusionskoeffizienten. Im folgenden wird die Massenerhaltung und ein elastisches Gleichgewicht, bei dem die Relaxationszeit für die Diffusion viel größer als für die Spannung ist, vorausgesetzt. Die gesamte Dehnung $\epsilon^{g e s}$ ergibt sich aus der Summe der einzelnen Dehnungen. Hier werden nur elastische $\epsilon^{e}$ und spanungsfreie Dehnungen $\epsilon^{c}$ berücksichtigt. Die spannungsfreie Dehnung kann durch Änderung der Zusammensetzung bei unterschiedlichem Atomvolumen oder durch unterschiedliche Ströme in ein Volumenelement entstehen. Im folgenden wird angenommen, daß sich die spannungsfreie Dehnung nur durch die Änderung des Atomvolumen mit der Konzentration ergibt. Zusammen 


\section{Interdiffusion}

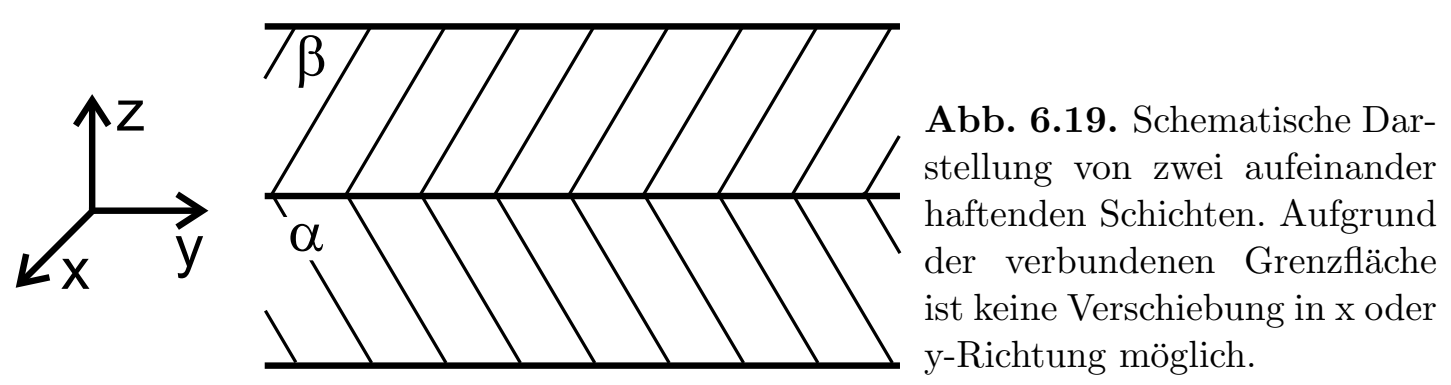

mit der Verzerrungsenergie, wie sie sich aus der Elastizitätstheorie ergibt, erhält man daraus folgendes Diffusionspotential:

$$
M(c, T)=M(c, 0)-\Omega\left(\frac{d E_{i j}^{c}}{d c} \cdot \sigma_{i j}+\frac{d s_{i j k l}}{d c} \cdot \sigma_{i j} \cdot \sigma_{k l}\right)
$$

mit $\Omega$ dem Atomvolumen, $\sigma$ dem Spannungstensor und $s_{i j k l}$ den elastischen Konstanten. Setzt man bei einem isotropen Festkörper die Vegardsche Regel an und nimmt an, daß die elastischen Konstanten konzentrationsunabhängig sind, dann ergibt sich ein Diffusionspotential mit einem Spannungsterm für einen isotropen Festkörper:

$$
M(c, T)=M(c, 0)-\Omega \eta \sigma_{k k}
$$

mit $\eta$ der Fehlpassungsparameter. Dieser ergibt sich für die Schichten $\alpha$ und $\beta$ wie folgt:

$$
\eta^{\alpha}=\frac{a_{\beta}-a_{\alpha}}{a_{\alpha}} \quad \text { und } \quad \eta^{\beta}=\frac{a_{\beta}-a_{\alpha}}{a_{\beta}} .
$$

Sind die Schichten an der Grenzfläche, wie in Abbildung 6.19 dargestellt, fest miteinander verbunden, so ist keine Verschiebung $\mathrm{u}$ in der $\mathrm{x}$ und $\mathrm{y}$-Ebene möglich. Aus $u_{x}=u_{y}=0$ ergibt sich für den Verzerrungstensor:

$$
\epsilon_{x x}=\epsilon_{y y}=0
$$

Da sich im Gleichgewicht die Kräfte der inneren Spannungen für jedes Volumenelement kompensieren, ergibt sich für den Spannungstensor:

$$
\sigma_{z z}=0 .
$$

Die Probe erfährt somit keine Verzerrung in $\mathrm{x}$ und $\mathrm{y}$-Richtung und ist in der z-Richtung spannungsfrei. Für den Verzerrungstensor ergibt sich in einem isotropen Festkörper mit einer von seiner Ausgangskonzentration $c_{0}$ abweichenden Zusammensetzung c

$$
\epsilon_{i k}=\frac{1+\nu}{E} \cdot \sigma_{i k}-\frac{\nu}{E} \cdot \sigma_{j j} \cdot \delta_{i k}+\underbrace{\eta \cdot\left(c-c_{0}\right) \cdot \delta_{i k}}_{\text {spannugsfreieDehnung } \epsilon_{i k}^{c}}
$$




\section{Interdiffusion}

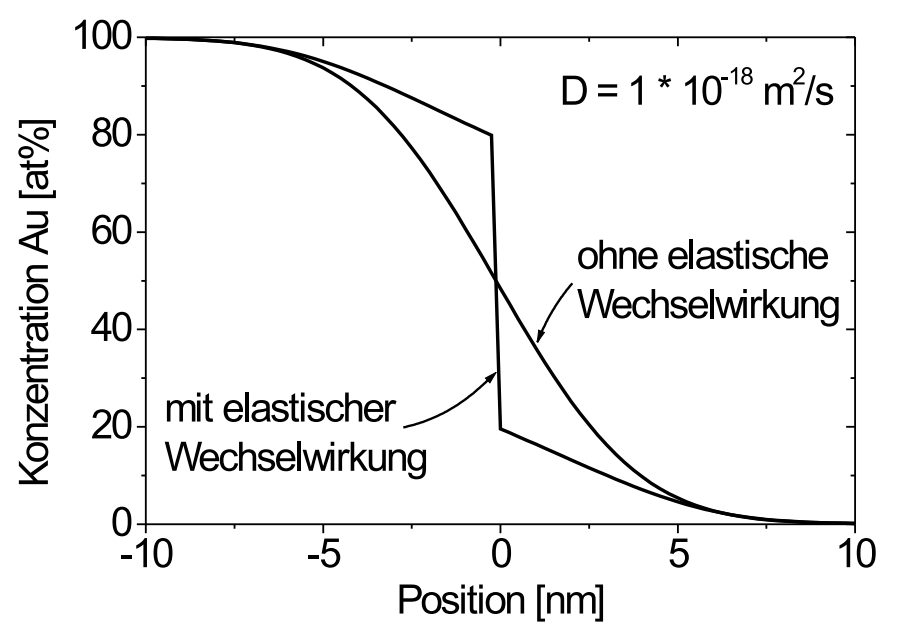

Abb. 6.20. Konzentrationsprofile unter dem Einfluß elastischer Spannungen an einer Grenzfläche zwischen Kupfer und Gold nach 5 Sekunden bei $430{ }^{\circ} \mathrm{C}$.

mit E dem Schubmodul und $\nu$ der Querkontraktionszahl. Damit ergibt sich für die Spannungen in $\mathrm{x}$ und $\mathrm{y}$-Richtung

$$
\sigma_{x x}=\sigma_{y y}=-\frac{E}{1-\nu} \cdot \eta \cdot\left(c-c_{0}\right)
$$

und damit für die Gesamtspannung mit $\Delta c=c-c_{0}$

$$
\sigma_{k k}=-\frac{2 E \eta \Delta c}{1-\nu}
$$

Für den Fluß unter Berücksichtigung der elastischen Spannungen mit einem isotropen Diffusionskoeffizienten folgt also:

$$
J_{z}=-\underbrace{B \cdot\left(\frac{d M(c, 0)}{d c}+\frac{2 \Omega \eta^{2} E}{1-\nu}\right)}_{D_{a p p}} \frac{d c}{d z}
$$

Für das chemische Potential M(c,0) wurde das Modell der ideale Lösung angesetzt. Damit ergeben sich die in Abbildung 6.20 dargestellten Lösungen der Gleichung 6.22 für einen intrinsischen Diffusionskoeffizienten von $1 \cdot 10^{-18} \mathrm{~m}^{2} / \mathrm{s}$ nach 5 Sekunden bei $430{ }^{\circ} \mathrm{C}$. Zur Berechnung wurden für jede Schicht die elastischen Konstanten von reinem Kupfer $\left(\mathrm{E}=1.1 \cdot 10^{11} \mathrm{~N} / \mathrm{m}^{2}, \nu=0.356\right.$ bei $\left.700{ }^{\circ} \mathrm{C}\right)$ und Gold $\left(\mathrm{E}=0.698 \cdot 10^{11} \mathrm{~N} / \mathrm{m}^{2}, \nu=0.428\right.$ bei $\left.430{ }^{\circ} \mathrm{C}\right)$ verwendet. Zusätzlich wurde eine Lösung der Gleichung 6.22 ohne den Term, der die elastischen Spannungen berücksichtigt, angegeben. An der Grenzfläche verursacht der unstetige Übergang des Spannungszustands, mit Zugspannungen im Gold und Druckspannungen im Kupfer, einen unstetigen Verlauf des chemischen Potentials und damit einen Konzentrationssprung. In den Schichten ergibt sich durch die elastische Spannung ein erhöhter Diffusionskoeffizient $D_{a p p}$. Dieser gleicht den kleineren Konzentrationsgradienten aus, so daß sich das Konzentrationsprofil entfernt von der Grenzfläche 


\section{Interdiffusion}

nicht mehr unterscheidet. Im Unterschied zur angegebenen Lösung kann man das reale Kupfer-Gold System nicht mit einer idealen Lösung beschreiben. Man findet einen erhöhten Interdiffusionskoeffizienten. Der entsprechende thermodynamische Faktor bewegt sich aufgrund der attraktiven Wechselwirkung zum Beispiel bei Goldkonzentration von 20 bis 40 at\% um einen Wert von 10 [105]. Die stark unterschiedlichen intrinsischen Diffusionskoeffizient und eine Abweichung von der idealen Lösung führen zu einem veränderten Konzentrationsverlauf, jedoch findet man in allen Fällen ein Konzentrationssprung an der Grenzfläche. Die für die Interdiffusion zwischen Kupfer und Gold ermittelten Konzentrationsprofile aus Abbildung 6.11 zeigen jedoch keinen Konzentrationssprung. Dies deutet darauf hin, daß die elastischen Spannungen zum großen Teil durch plastische Relaxation abgebaut wurden. Wie im vorangegangenen Kapitel gezeigt wurde, erfolgt die plastische Verformung durch das Klettern von Frank-Partialversetzungen und das Gleiten von Shockley-Partialversetzungen. Beide Prozesse laufen auf den gemeinsamen (11) $\overline{1}$ ) Ebenen von Kupfer und Gold ab. Jedoch beträgt der gefundene Winkel zwischen den Burgersvektoren der Frank-Partialversetzung und einer Shockley-Partial immer $90^{\circ}$. Die plastische Relaxation erfolgt damit in unterschiedliche Richtungen.

Mit der fortschreitenden Interdiffusion wirken Kräfte auf die Versetzungen. Eine Analyse dieser Wechselwirkungen kann aufgrund der Arbeiten von Hirth et al. durchgeführt werden [97]. Während die Diffusionszone gebildet wird, wirken drei wesentliche Kräfte auf die Versetzungen. Aufgrund der unterschiedlichen Atomvolumina von Kupfer und Gold wird ein Spannungsfeld aufgebaut. Das Spannungsfeld erzeugt eine Peach-Köhler Kraft auf die Versetzungen

$$
F_{\sigma}=\sigma \cdot b
$$

mit $\sigma$ dem Spannungsfeld und b dem Burgersvektor der Versetzung. Aufgrund der Peach-Köhler Kraft werden die Versetzungen von der Grenzfläche nach beiden Seiten in die Diffusionszone getrieben. Durch die von ihnen verursachte plastische Verformung wird die Spannung relaxiert. Dabei tragen die beiden gefundenen Versetzungstypen zur Relaxation der Spannungen bei. Jedoch ist die Geschwindigkeit, mit der sich die Versetzungen fortbewegen können höchst unterschiedlich. So kann die Shockley-Partialversetzung mit der materialspezifischen Schallgeschwindigkeit gleiten und damit wohl instantan dem Konzentrationsprofil folgen. Die Frank-Partialversetzung kann dagegen nur klettern, was mit der gleichen Geschwindigkeit wie der Materialtransport erfolgt. Die Versetzungsbewegung der Frank-Partialversetzung kann damit einen ganz anderen Einfluß auf die Kinetik der Interdiffusion haben als die Shockley-Partialversetzung.

Das Klettern der Frank-Partialversetzung führt zur Bildung und Vernichtung von Leerstellen, je nachdem in welche Richtung sie sich bewegen. Dies und die unterschiedlichen Diffusionsgeschwindigkeiten verursachen ein Veränderung der Leerstellenkonzentration weg vom Gleichgewichtszustand. Da in diesem Fall keine 


\section{Interdiffusion}

Konzentrationsprofil

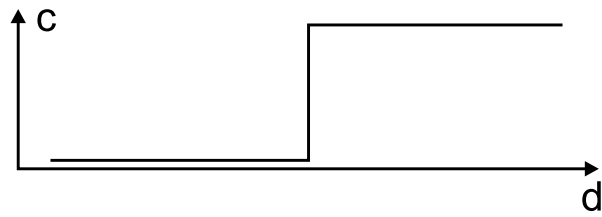

(111) Ebenen

$\mathrm{Au}$

$\mathrm{Cu}$

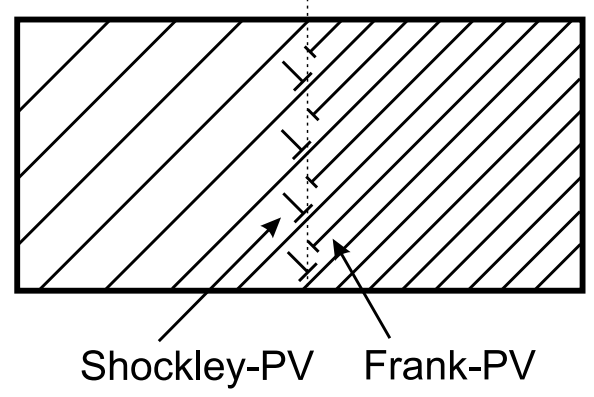

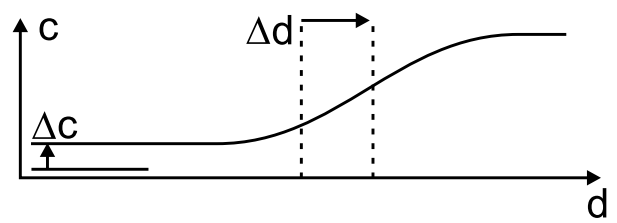

Reaktion

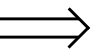

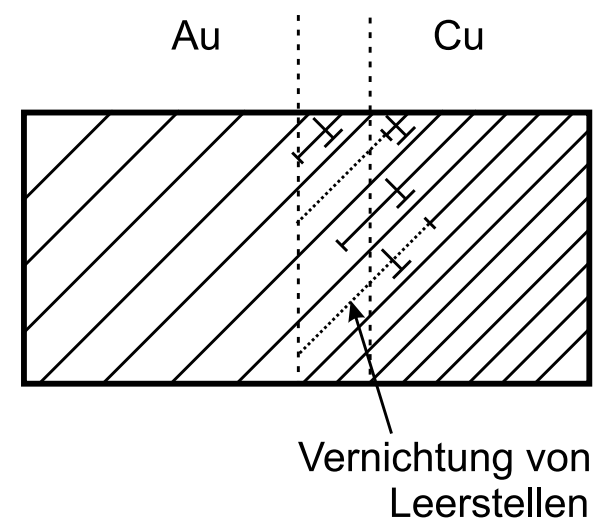

Abb. 6.21. Schematische Darstellung des Zusammenhangs zwischen dem Konzentrationsprofil und der Versetzungsstruktur vor und nach einer Wärmebehandelung.

weiteren Leerstellensenken und Quellen, wie Oberflächen oder Großwinkelkorngrenzen, vorliegen, kann man davon ausgehen, daß die Leerstellenkonzentration an den Versetzungen ausgeglichen wird, die in hoher Dichte an der Grenzfläche vorliegen. Für das chemische Potential der Leerstellen

$$
\Delta \bar{G}=k T \ln \left(\frac{c_{l}}{c_{l}^{0}}\right)
$$

mit $c_{l}$ und $c_{l}^{0}$ der aktuellen und der Gleichgewichtsleerstellenkonzentration, ergibt sich nach Hirth und Lothe [41] die folgende osmotische Kraft auf die Stufenversetzungen:

$$
\begin{aligned}
F_{o s} & =-\frac{b \Delta \bar{G}}{\Omega} \\
& =-\frac{k T b}{\Omega} \ln \left(\frac{c_{l}}{c_{l}^{0}}\right)
\end{aligned}
$$

mit $\Omega$ dem Atomvolumen. Da die Shockley-Partialversetzung gleitet und dabei keine Leerstellen erzeugt oder vernichtet, erfährt sie auch keine Kraft aufgrund eines Leerstellenungleichgewichts. Die höhere Diffusionsgeschwindigkeit des Kupfers führt zu einem dem Kupferstrom entgegengesetzten Leerstellenstrom und so zu einer Anreicherung von Leerstellen in der Kupferschicht. Durch das Klettern der zusätzlichen Ebenen des Kupfers in die Goldschicht hinein werden Leerstellen 


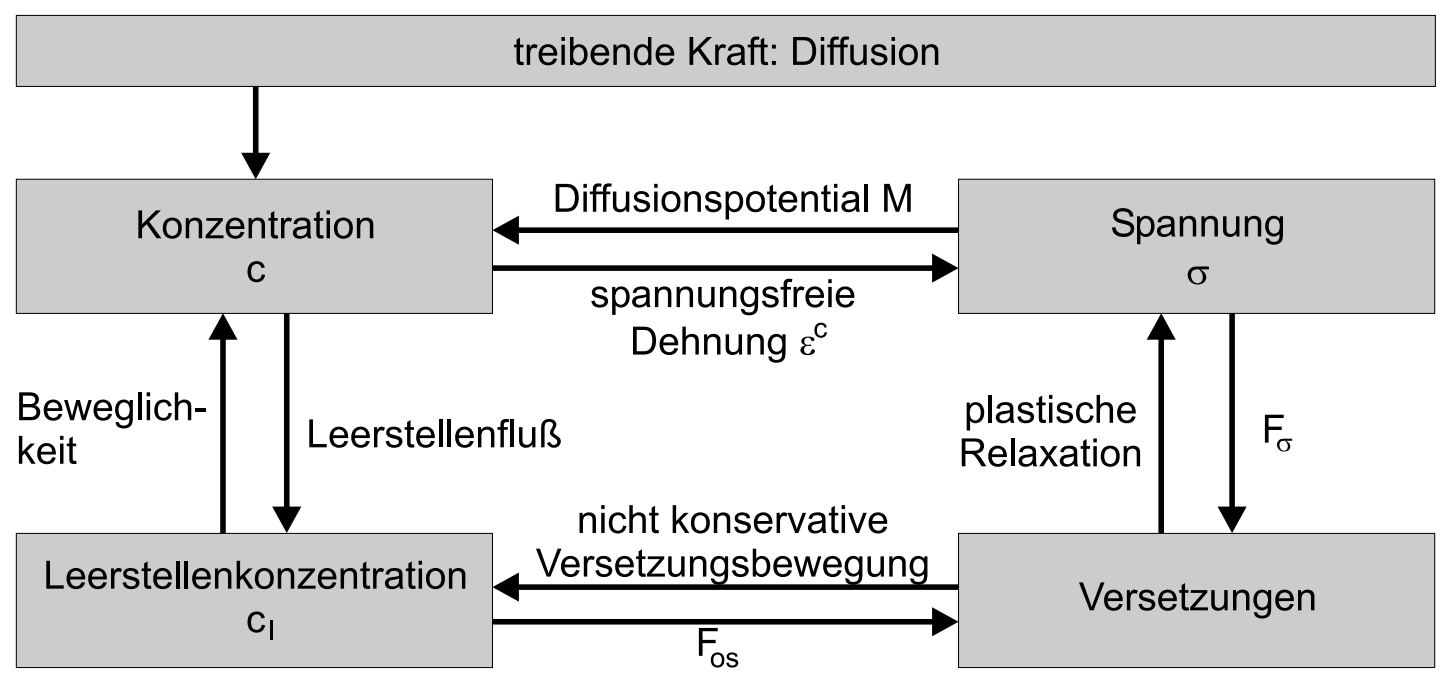

Abb. 6.22. Schematische Darstellung der verschiedenen Abhängigkeiten zwischen der Konzentration, der Leerstellenkonzentration, der Spannung und den Versetzungen unter den Randbedingungen in epitaktischen Kupfer/Gold Schichten.

erzeugt, ein Klettern der Ebenen zurück in die Kupferschicht vernichtet Leerstellen. Damit baut dieser Prozess, im Gegensatz zum Leerstellenstrom durch die Diffusion, eine Leerstellenübersättigung in der Goldschicht und einem Leerstellenuntersättigung in der Kupferschicht auf. Jedoch kann ohne quantitative Rechnungen keine Aussage getroffen werden, welcher der beiden Prozesse dominiert.

Aufgrund ihrer Struktur findet man bei den eingeschobenen Ebenen jeweils zwei Stapelfehler. Die Stapelfehlerenergie sorgt für eine dritte Kraft auf die Versetzungen, die die eingeschobenen Ebenen wieder an die ursprüngliche Grenzfläche treibt. Aufgrund der niedrigen Stapelfehlerenergie von $40 \mathrm{~mJ} / \mathrm{m}^{2}$ im Gold und $60 \mathrm{~mJ} / \mathrm{m}^{2}$ im Kupfer [35] dürfte dieser Beitrag in der Kräftebilanz klein sein. Sie wird im folgenden vernachlässigt.

Aus dem Vergleich der Struktur mit der Diffusionszone in Abbildung 6.11 geht hervor, daß die Defekte tatsächlich von der dominierenden Peach-Köhler Kraft über den größten Teil Diffusionszone verteilt sind. Aufgrund der Asymmetrie in den Beweglichkeiten verschiebt sich das Diffusionsprofil in Richtung der Kupferschicht, so daß Leerstellen in der Diffusionszone in großer Zahl vernichtet werden. Dieser Vorgang ist in Abbildung 6.21 noch einmal schematische dargestellt. Es liegt daher nahe, daß die Interdiffusion im Reaktionsschritt I durch Leerstellenverarmung zum erliegen kommt. Jedoch ist, aufgrund der unterschiedlichen, miteinander verknüpften Kräfte die Reaktion sehr komplex.

In Abbildung 6.22 ist abschließend das komplexe Beziehungsgeflecht zwischen der Konzentration, der Leerstellenkonzentration, den entstehenden Spannungen und 


\section{Interdiffusion}

der Versetzungsbewegung schematisch dargestellt. Auf den dargestellten Beziehungen zwischen den verschiedenen Größen muß eine quantitative Beschreibung aufbauen. Eine Computersimulationen ist jedoch aufgrund der kleinen Leerstellenkonzentrationen und der komplexen Struktur der gekoppelten Differentialgleichungen auch numerisch ein sehr anspruchsvolles Problem [93], so daß diese Arbeit die quantitative Lösung dieses Diffusionsproblems schuldig bleiben muß. Eine entsprechende Ausarbeitung ist jedoch in Vorbereitung [40].

\subsubsection{Diffusionsinduzierte Neubildung des Gefüges}

Nach dem Verharren der Interdiffusion im ersten Schritt wird die Reaktion nach kurzer Zeit fortgesetzt, wie die SNMS Ergebnisse in Abbildung 6.8 zeigen. Auch die Röntgenbeugungsuntersuchung in Abbildung 6.9 zeigt neue Reflexe zwischen den (111) Reflexen von Kupfer und Gold. Dies kann auf die Bildung neuer Körner einer Mischkonzentration zurückgeführt werden. Eine elektronenmikroskopische Charakterisierung zeigt, daß sich in den Schichten tatsächlich ein neues Gefüge mit Körnern, die eine homogene Mischkonzentration aufweisen, gebildet hat. Exemplarisch wurde eine Hellfeld- und eine Z-Kontrastabbildung eines typischen Reaktionszustands nach 10 Sekunden bei $430{ }^{\circ} \mathrm{C}$ in Abbildung 6.23 dargestellt. In der Bildmitte ist an der Grenzfläche noch die durch die Interdiffusion in der Frühphase gebildete Defektstruktur zu sehen. Am linken und rechten Bildrand jedoch erkennt man das neugebildete Gefüge. Die neuen Körner weisen dabei verschiedene Orientierungen auf und sind durch Großwinkelkorngrenzen voneinander
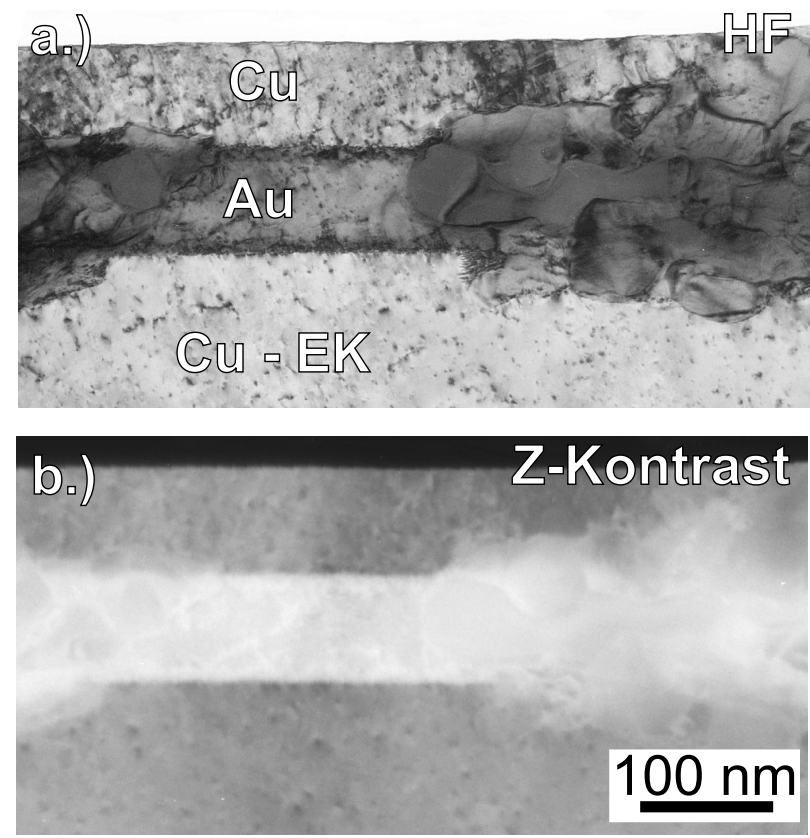

Abb. 6.23. (a) Hellfeld und (b) ZKontrastaufnahme (144 mrad) eines für 10 Sekunden ausgelagerten $\mathrm{Cu} / \mathrm{Au}$ Schichtpakets mit einer Einzelschichtdicke von $150 \mathrm{~nm}$ auf einem Kupfereinkristall. In der Schichtstruktur bildet sich eine neue Kornstruktur, wobei die einzelnen Körner eine Mischkonzentration aufweisen. 


\section{Interdiffusion}

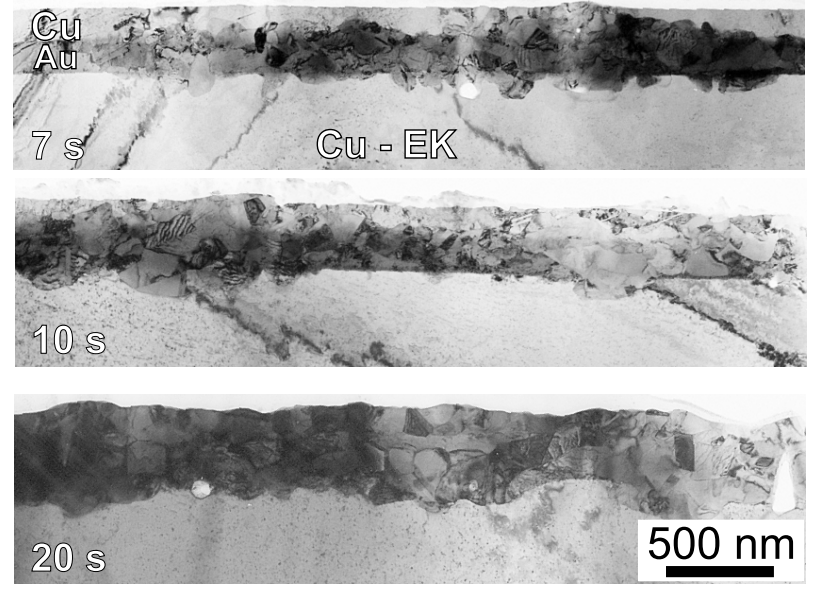

Abb. 6.24. Hellfeldaufnahme einer bei $430{ }^{\circ} \mathrm{C}$ für 7,10 und 20 Sekunden ausgelagerten $\mathrm{Cu}(150 \mathrm{~nm}) / \mathrm{Au}(150 \mathrm{~nm})$ Schichtprobe auf einem Kupfereinkristall. Mit zunehmender Auslagerungszeit nimmt der Anteil der unreagierten Grenzfläche ab. Volumentransportprozesse werden mit zunehmender Auslagerung durch eine Oberflächenaufrauhung sichtbar.

getrennt. In der Z-Kontrastaufnahme kann man zusätzlich noch die unregelmäBige chemische Zusammensetzung der neu gebildeten Körner erkennen und an einigen Stellen unstetige Konzentrationssprünge an den Korngrenzen nachweisen. In Abbildung 6.24 sind weitere Auslagerungszustände abgebildet. Das neu gebildete Gefüge erstreckt sich mit zunehmender Auslagerungszeit über einen immer größeren Bereich der Grenzfläche. Nach 20 Sekunden hat sich im gesamten Bereich der Schichten ein neues Korngefüge gebildet. Tendenziell liegt die neu gebildete Kornstruktur in zusammenhängenden Regionen vor. Die Korngröße ist im Vergleich zur ursprünglichen Schichtdicke deutlich reduziert. Betrachtet man die des Schichtpakets in Abbildung 6.24 nach 20 Sekunden, so erkennt man den Volumentransport während der Bildung des neuen Korngefüges anhand der deformierten Oberfläche. Der wesentliche Reaktionsmechanismus und die Kinetik in dieser zweiten Phase ist somit durch die Bildung des neuen Gefüges bestimmt.

Eine genaue Analyse der SNMS Messungen aus Abbildung 6.8 liefert weitere Einblicke in den Reaktionsmechanismus. Betrachtet man den Kernbereich der Schichten, so findet man nach einer Inkubationszeit von wenigen Sekunden eine kontinuierliche Anreicherung der jeweils anderen Komponente. In Abbildung 6.25 sind diese Anreicherungen jeweils für die einzelnen Schichten dargestellt. Danach kann die Kinetik der Reaktion in drei Bereiche eingeteilt werden. In Abschnitt I verharrt die Reaktion praktisch im metastabilen Zustand der ersten Interdiffusionsphase. Im Bereich II findet man ein extrem schnelles Fortschreiten der Reaktion, während im Abschnitt III die Reaktionsgeschwindigkeit wieder deutlich abfällt.

Eine Abschätzung der Zusammensetzung der neu entstehenden Körner kann aus der Beugungsuntersuchung gewonnen werden. Dazu wird angenommen, daß in der neuen Kornstruktur die Spannungen weitgehend relaxiert sind. Unter diesen Umständen kann aufgrund des Gitterparameters auf die Zusammensetzung der 


\section{Interdiffusion}
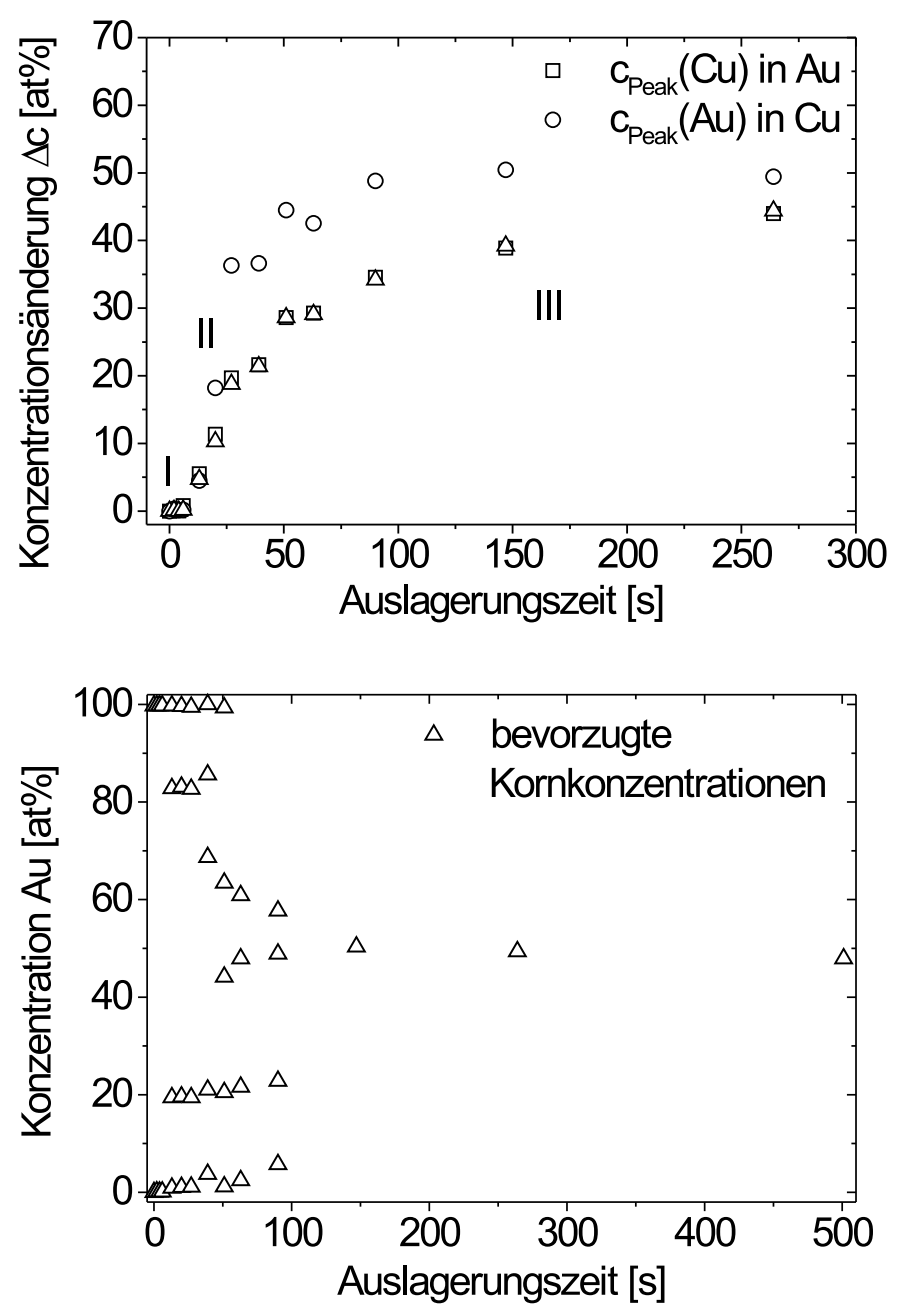

Abb. 6.25. Konzentrationsänderung in der Mitte der verschiedenen Schichten eines $\mathrm{Au}(60 \mathrm{~nm}) / \mathrm{Cu}(60 \mathrm{~nm})$ / $\mathrm{Au}(60 \mathrm{~nm})$ Schichtpakets auf einem Kupfereinkristall. Die Konzentrationsänderung durch die Interdiffusion in der Frühphase wurde abgezogen. Die Kinetik der Reaktion läßt sich dann in 3 Bereiche einteilen.

Abb. 6.26. Konzentrationsänderung in der Mitte der verschiedenen Schichten eines $\mathrm{Au}(150 \quad \mathrm{~nm}) / \mathrm{Cu}(150 \mathrm{~nm}) /-$ $\mathrm{Au}(150 \mathrm{~nm}) \quad$ Schichtpakets auf einem Kupfereinkristall. Die Konzentrationsänderung durch die Interdiffusion in der Frühphase wurde abgezogen.

neu entstandenen Kornstruktur geschlossen werden. Die so ermittelten Konzentrationen sollten aufgrund der weitreichenden Annahmen vorsichtig interpretiert werden, sie können jedoch zumindest einen Hinweis auf die chemische Zusammensetzung des neu gebildeten Gefüges geben. Aus den in Abbildung 6.9 dargestellten Röntgenbeugungsuntersuchungen wurden die Positionen der Peaks entnommen. Über die Vegardsche Regel, die im ungeordneten Kupfer-Gold System recht gut erfüllt ist, wurden die ermittelten Gitterabstände in Konzentrationen umgerechnet und in Abbildung 6.26 dargestellt. Man findet für die zuerst gebildeten Körner eine Konzentration von 20 at\% bzw. 85 at\% Gold. Mit zunehmender Auslagerungszeit findet dann eine Umlösung in Körner mit einer Goldkonzentration von 40-60 at\% statt und es stellt sich langsam die durch die Schichtdicken vorgegebene Gleichgewichtskonzentration ein.

Zur Analyse der Kinetik der Gefügeneubildung wird die Johnson-Mehl-Avrami (JMA) Kinetik [3,44] herangezogen. Dieses Modell wird häufig zur Beschreibung 


\section{Interdiffusion}

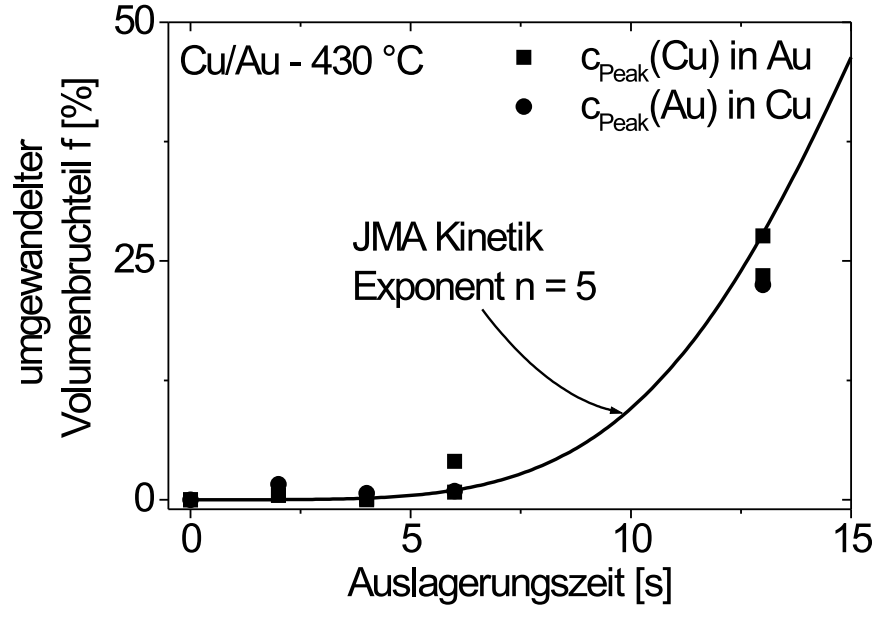

Abb. 6.27. Anpassung einer JMA Kinetik an den umgewandelten Volumenbruchteil eines $\mathrm{Au}(60 \mathrm{~nm}) / \mathrm{Cu}(60 \mathrm{~nm})$ / $\mathrm{Au}(60 \mathrm{~nm})$ Schichtpakets auf einem Kupfereinkristall. Es ergibt sich ein JMA Exponent $n$ von 5 .

einer Keimbildungs- und Wachstumskinetik verwendet, jedoch ergeben die ermittelten Parameter keinen eindeutigen Hinweis auf die Art der Reaktion. In diesem Fall scheint die Anwendung jedoch gerechtfertigt, da ausreichende Daten über die Mikrostruktur vorhanden sind, um die Ergebnisse richtig zu interpretieren. Bei der JMA Kinetik wird der Bruchteil des umgewandelten Volumens $\mathrm{f}$ in einem kinetischen Gesetz

$$
f=1-\exp \left(-k \cdot t^{n}\right)
$$

beschrieben, wobei $\mathrm{n}$ die Keimbildungs- und Wachstumsbedingungen und $\mathrm{k}$ die Reaktionsrate beschreibt. Geht man von einer Konzentration der neu gebildeten Körner von $c_{\text {Korn }}=20$ at\% aus, wie es die Röntgenbeugungsuntersuchungen nahelegen, so ergibt sich der umgewandelte Volumenbruchteil für frühe Phasen der Gefügeneubildung aus $f=c_{P e a k} / c_{K o r n}$. Unter diesen Umständen kann so für die Frühphasen der Reaktion der umgewandelte Bruchteil abgeschätzt werden. Eine Anpassung an die aus den SNMS-Untersuchungen ermittelten Daten ist in Abbildung 6.27 dargestellt. Um die Inkubationszeit zu reproduzieren ist ein JMA Exponent $\mathrm{n}=5$ erforderlich, unabhängig vom genauen Wert von $c_{K o r n}$. Für eine Reaktion, die durch die Bildung von Ausscheidungen an der Grenzfläche kontrolliert wird, bedeutet ein Exponent von über 4 einen Anstieg der Keimbildungsrate $[11,13]$. Für unseren Fall bedeutet dies, daß der erste Keim an der epitaktischen Grenzfläche schwierig zu bilden ist. Der Aufbau eines wachstumsfähigen Keims wird durch langsamen Transport in der Grenzflächenregion, die eine Verarmung an Leerstellen aufweist, stark behindert und bestimmt die Kinetik im Bereich I. Auch erschweren bei der Keimbildung entstehende elastische Spannungen ein Wachstum über die kritische Keimbildungsgröße. Ist die Bildung eines wachstumsfähigen ersten Keims dagegen erst einmal geglückt, erfolgt der 


\section{Interdiffusion}

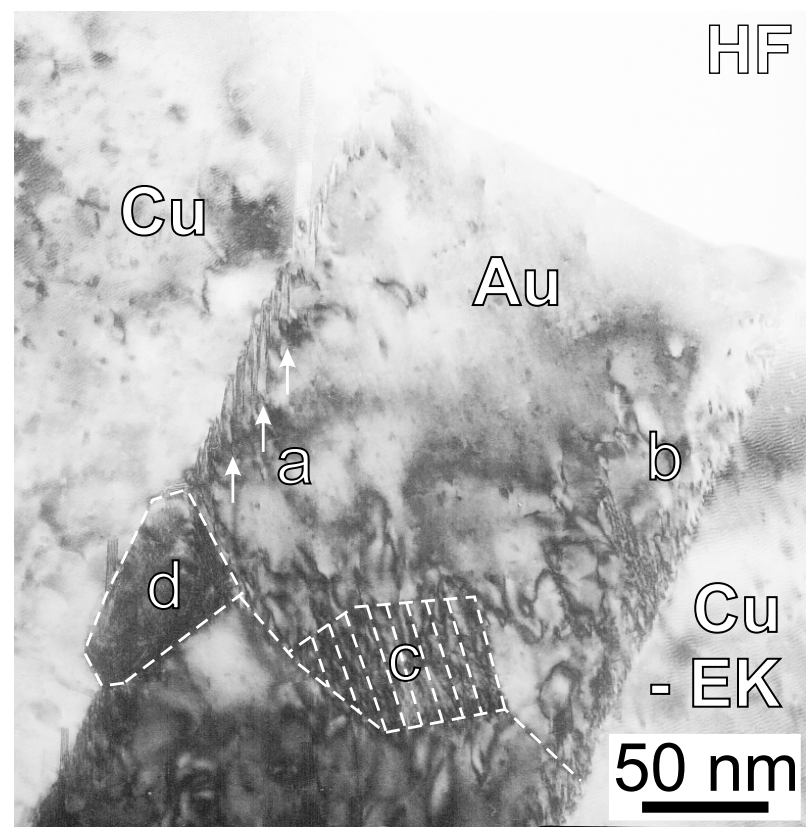

Abb. 6.28. Hellfeldaufnahme eines für 5 Sekunden bei $430{ }^{\circ} \mathrm{C}$ ausgelagerten Kupfer/Gold Schichtpakets mit einer Einzelschichtdicke von $50 \mathrm{~nm}$ auf einem Kupfereinkristall. An der Zwillingsgrenzfläche findet man noch die Defektstruktur des Frühstadiums (a), während an der direkt epitaktischen Grenzfläche (b) keine auffälligen Defekte zu sehen sind. An einem Tripelpunkt, an dem die Kleinwinkelkorngrenze (c) endet, ist ein neu gebildeter Keim (d) erkennbar.

Transport an der neu gebildeten Großwinkelkorngrenze dagegen sehr viel schneller. Auch die Bildung weiterer Körner ist dort stark erleichtert und man findet eine sehr schnelle Reaktion im Abschnitt II.

Die unterschiedlichen elastischen Eigenschaften der Schichten wirken sich dann auch in der Keimbildungsphase aus. In den elektronenmikroskopischen Aufnahmen ist zumeist die Reaktion in der Goldschicht weiter fortgeschritten. Da das Gold elastisch weicher ist, können dort einfacher neue Körner gebildet werden als in der Kupferschicht. Zusätzlich beobachtet man in den SNMS Messungen, daß die Keimbildungsbarriere in einem Mehrfachschichtpaket mit zunehmender Tiefe größer wird. So findet man bei einem Schichtpaket mit der Schichtreihenfolge $\mathrm{Au} / \mathrm{Cu} / \mathrm{Au} / \mathrm{Cu} / \mathrm{Au}$ auf einem Kupfereinkristall eine Behinderung der Keimbildung ab der vierten Schicht, in diesem Fall der Kupferschicht. Während die Reaktion in den ersten drei Schichten schon begonnen hat, erhält man keine Veränderung des Konzentrationsprofils in den beiden untersten Schichten. Eine Keimbildung wird dort durch die veränderten elastischen Eigenschaften unterdrückt. Bis zur 3. Schicht scheint die Nähe der Oberfläche die Relaxation der elastischen Spannungen der gebildeten Keime zu vereinfachen. Zusätzlich sieht man eine schnellere Schichtreaktion in der fünften, weicheren Goldschicht, verglichen mit der vierten, härteren Kupferschicht. Dies ist konsistent mit der Beobachtung, daß auch in den obersten Lagen die Gefügeneubildung in den Goldschichten schneller als in den umliegenden Kupferschichten stattfindet.

Eine Keimbildung an der epitaktischen Grenzfläche ist in Abbildung 6.28 gezeigt. An der Grenzfläche (a) findet man noch die durch die Interdiffusion gebildete metastabile Defektstruktur. An einem Tripelpunkt zwischen einer durch 


\section{Interdiffusion}

die Goldschicht laufenden Kleinwinkelkorngrenze (c) und der Grenzfläche findet man einen neu gebildeten Keim (d). Es scheint, daß die Keimbildung bevorzugt an ausgezeichneten Keimbildungszentren stattfindet wie zum Beispiel solchen Tripelpunkten. Dort ist aufgrund der vorhandenen Kleinwinkelkorngrenze ein schnellerer Materialtransport möglich, was die Bildung eines Keims erleichtert. Der neu gebildete Keim ist mit einer Korngrenze umgeben, wodurch der Materialtransport und die Bildung von neuen Körnern erleichtert wird. Dies stimmt mit der Beobachtung überein, daß die Regionen mit der neuen Kornstruktur relativ große, zusammenhängende Gebiete bilden.

Nachdem die Kornstruktur gebildet wurde, wird durch weitere Keimbildung von Körnern mit einer jeweils höheren Mischkonzentration und einer langsamen Konzentrationsänderung in bereits bestehenden Körnern eine Gleichgewichtskonzentration eingestellt. Diese langsamere Kinetik kennzeichnet den Abschnitt III in Abbildung 6.27. Wie schon in Abbildung $6.24 \mathrm{zu}$ sehen ist, führt dieser Prozeß auch zu einer massiven Verformung der Reaktionszone.

\subsubsection{Aktivierungsenergie und Bildungsenthalpie für die Neubildung des Gefüges}

Um den atomaren Prozeß genauer zu charakterisieren und die Reaktionsenthalpien zu bestimmen, wurden DSC Untersuchungen durchgeführt. Dabei wird davon ausgegangen, daß die Durchmischung durch einen Kornbildungsprozeß auch bei niedrigen Temperaturen dominant ist. Daß diese Annahme gerechtfertigt ist, ergibt sich aus den im nächsten Kapitel dargestellten Ergebnissen. Dazu wurden dünne, freistehende Kupfer/Gold Schichtpakete mit einer Einzelschichtdicke von jeweils 25 oder $50 \mathrm{~nm}$ bei verschiedenen Heizraten ausgemessen. Das Ergebnis dieser Untersuchung ist in Abbildung 6.29 dargestellt. Beim ersten Aufheizen reagieren die Schichten miteinander und man erhält eine positive Wärmetönung (a) mit einem Maximum bei der Temperatur $T_{p}$. Je nach Heizrate ist die Reaktion beim Ordnungsübergang von etwa $400{ }^{\circ} \mathrm{C}$ schon abgeschlossen. Bei hohen Heizraten findet man fast keine Wärmetönung beim ersten Ordnungsübergang, während im zweiten Aufheizvorgang praktisch kein Unterschied zwischen verschiedenen Heizraten erkennbar ist. Im wesentlichen sind drei Einflüsse auf die Reaktion zu erkennen. Erstens ist die Ordnung nur langsam und in jedem Fall erst nach der Durchmischung möglich. Bei hohen Heizraten ist die Durchmischung beim Erreichen der Ordnungstemperatur $T_{c}$ nicht abgeschlossen und es ergibt sich nur eine kleine Wärmetönung durch den Entordnungsvorgang. Zweitens bleibt bei kleinen Heizraten zunächst ein Spektrum von Kornzusammensetzungen, weshalb sich ein sehr breiter Entordnungspeak im ersten Durchlauf ergibt. Drittens ist die Durchmischung nach dem ersten Aufheizen bis auf $500{ }^{\circ} \mathrm{C}$ vollständig abgeschlossen. 


\section{Interdiffusion}

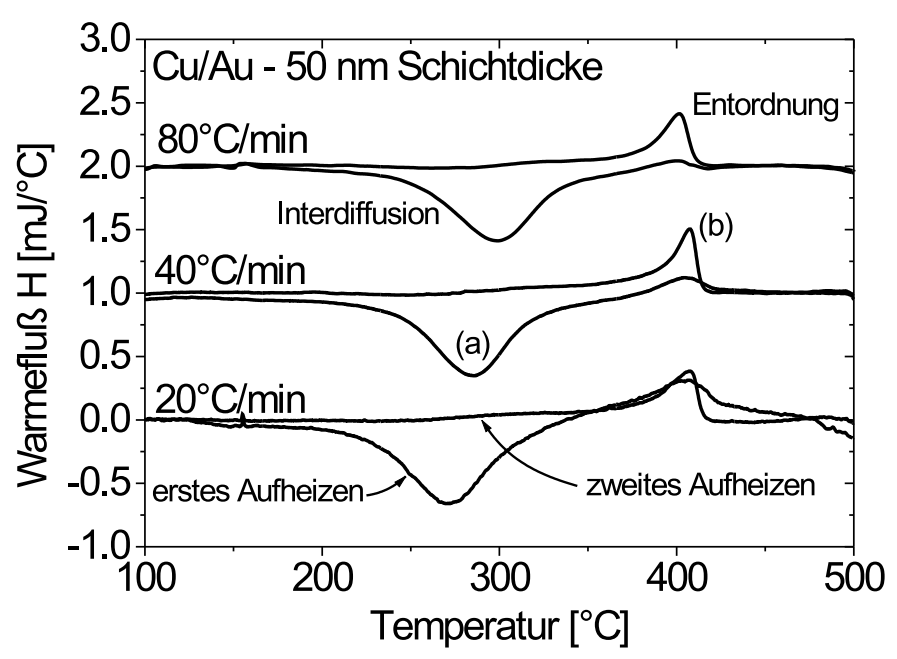

Abb. 6.29. DSC Messung eines $\mathrm{Cu} / \mathrm{Au}$ Multischichtpakets mit einer Einzelschichtdicke von $30 \mathrm{~nm}$ bei Heizraten von 20,40 und $80 \mathrm{~K} / \mathrm{min}$. Die in einem ersten Durchlauf frei werdende Energie des Peaks (a) kommt von der Interdiffusion der Schichten. Der Peak (b) wurde durch den Ordnungsübergang erzeugt. Im zweiten Aufheizdurchgang findet man nur noch den Peak (b) am Ordnungsübergang.

Deshalb erhält man beim zweiten Durchlauf immer den gleichen Entordnungspeak bei gleicher Temperatur.

Aus den Messungen ergibt sich für die Interdiffusion ein Energiegewinn von 40.44 $\mathrm{J} / \mathrm{g}$, die Ordnungseinstellung liefert einen Energiegewinn von $13.64 \mathrm{~J} / \mathrm{g}$. Damit liefert die Interdiffusion den größten Energiebeitrag und ist die treibende Kraft für die Reaktion. Aus der Verschiebung der Peaktemperatur $T_{p}$ kann man nach Kissinger [50,51] die Aktivierungsenergie des atomaren Prozesses bestimmen. Dafür benutzt man die folgende Abhängigkeit:

$$
\frac{d\left(\ln \frac{\Phi}{T_{p}^{2}}\right)}{d\left(\frac{1}{T_{p}}\right)}=\frac{E}{k}
$$

wobei $\Phi=\mathrm{dT} / \mathrm{dt}$ die Heizrate, $T_{p}$ die Temperatur maximaler Wärmetönung für eine Heizrate, E die Aktivierungsenergie des atomaren Prozesses und $\mathrm{k}$ die Boltzmannkonstante bezeichnen. Eine Auftragung von $\ln \left(\Phi / T_{\text {Peak }}^{2}\right)$ über $1 / T_{\text {Peak }}$ ergibt eine Gerade mit der Steigung E/k und damit die Aktivierungsenergie E. Aus der Kissingerauftragung in Abbildung 6.30 für die Schichten mit einer Einzelschichtdicke von $50 \mathrm{~nm}$ ergibt sich eine Aktivierungsenergie von $1.19 \mathrm{eV}$, eine Analyse der Schichten mit einer Einzelschichtdicke von $25 \mathrm{~nm}$ liefert einen Wert von $1.17 \mathrm{eV}$. Im Mittel erhält man somit eine Aktivierungsenergie von $1.18 \mathrm{eV}$.

Diese niedrigen Aktivierungsenergien deuten auf eine Korngrenzdiffusion hin. Im massiven Kupfer und Gold findet man für die Korngrenzdiffusion Aktivierungsenergien zwischen 0.87 und $1.09 \mathrm{eV}[2,12]$, für die Volumendiffusion einen Wert von etwa $2 \mathrm{eV}[29,109]$. Aleshin et al. [1] finden in dünnen Schichten niedrige Aktivierungsenergien von $0.94 \mathrm{eV}$ und $0.98 \mathrm{eV}$ für die Korngrenzdiffusion und 


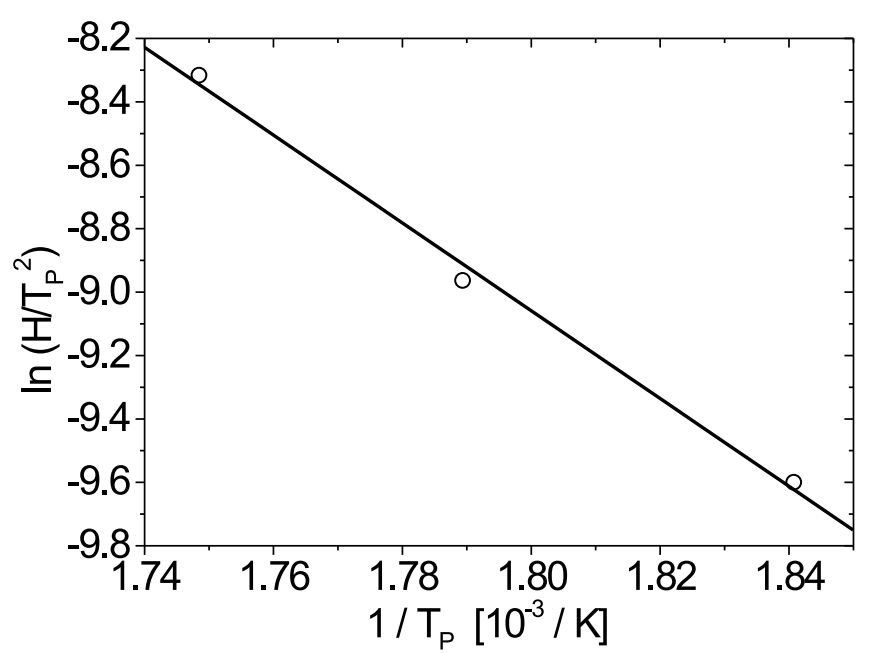

Abb. 6.30. Kissingerauftragung zur Bestimmung der Aktivierungsenergie des Reaktionsprozesses bei der Interdiffusion von $\mathrm{Cu} / \mathrm{Au}$ Schichten. Für die Schichten mit der $50 \mathrm{~nm}$ Einzelschichtdicke ergibt sich eine Aktivierungsenergie von $1.19 \mathrm{eV}$.

$1.10 \mathrm{eV}$ für die Volumendiffusion. Sie schreiben diese niedrige Aktivierungsenergie einer hohen Zahl struktureller Defekte in den untersuchten Schichten zu. Da zu einem relativ frühen Zeitpunkt der Reaktion das Korngefüge komplett neu gebildet wird und so die hohe Defektdichte der Schichten reduziert werden kann, deutet die niedrige Aktivierungsenergie im Lichte der hier vorgestellten mikroskopischen Analysen eher auf einen von Korngrenzdiffusion getragenen Prozess hin.

\subsubsection{Zusammenfassung des Reaktionsverlaufs}

Aus den hier dargestellten Ergebnissen ergibt sich das folgende Bild des Reaktionsverlaufs. Nach der Bildung eines wachstumsfähigen Keims mit einer Großwinkelkorngrenze werden neue Körner an diesem Keim gebildet. Eine Reaktionsfront, an der die neuen Körner gebildet werden, läuft durch das unreagierte Gebiet. Durch die hohe Geschwindigkeit der Reaktionsfront kann nur eine begrenzte Zahl von Atomen der jeweils anderen Komponente herantransportiert und in die neue Kornstruktur eingebaut werden. Damit erhält man ein goldreiches Gefüge an der Position der ehemaligen Goldschicht und ein kupferreiches Gefüge in der ehemaligen Kupferschicht. Mit den Röntgendiffraktometermessungen kann der Gehalt auf etwa 15-20 at\% abgeschätzt werden. Aufgrund der nicht vollständigen Durchmischung wird weiteres Material über die Korngrenzen transportiert. Zur weiteren Durchmischung scheinen zwei Prozesse abzulaufen. Zum einen werden aufgrund der niedrigen Konzentration weiter Keime höherer Konzentration in der neuen Kornstruktur gebildet, wie dies aus den Röntgenbeugungsuntersuchungen hervorgeht. Zum anderen scheint ein Prozeß, der in der Literatur als diffusionsinduzierte Korngrenzwanderung bezeichnet wird, abzulaufen [18, 124]. Dabei werden durch die Korngrenzdiffusion weitere Atome der anderen Komponente in das neu gebildete Gefüge transportiert. Diese dringen in die Körner über 


\section{Interdiffusion}

die normale Volumendiffusion ein. Die Fehlpassung zwischen Kupfer und Gold sorgt dabei für die Induzierung von elastische Spannungen im Material. Befindet sich auf einer Seite der Korngrenze ein Korn mit einem kleineren Spannungszustand, zum Beispiel durch die Relaxation mittels eingebauter Versetzungen oder einer höheren Konzentration, so wird eine Kraft auf die Korngrenze ausgeübt und die Korngrenze verschiebt sich in Richtung des im größeren Spannungszustand befindlichen Korns. Es ist leicht einzusehen, daß die diffusionsinduzierte Korngrenzwanderung mit einem starken Volumentransport gerade in einem System mit hoher Gitterfehlpassung wie Kupfer-Gold verbunden ist wie dies in den hier vorgestellten Experimenten zu beobachten ist.

\subsection{Diskussion und Vergleich mit anderen Experimenten}

Wie gezeigt ist der Ablauf der Interdiffusion in den Systemen Kupfer-Gold und Silber-Gold sehr verschieden. Im epitaktischen Kupfer-Gold Schichtsystem kam durch die große Gitterfehlpassung die Interdiffusion in einem ersten Reaktionsschritt aufgrund eines Leerstellenmangels zum Erliegen. Anschließend wurde in einem zweiten Reaktionsschritt ein neues Korngefüge gebildet, das sich mittels weiterer Keimbildung und diffusionsinduzierter Korngrenzenwanderung der Gleichgewichtskonzentration nähert.

Bei den epitaktischen Silber-Gold Schichten sind wegen der geringen Gitterfehlpassung nur sehr wenige Misfitversetzungen eingebaut. Die intrinsischen Diffusionskoeffizienten der beiden Elemente unterscheiden sich nur um den Faktor $D_{A g} / D_{A u} \approx 2.5$ [57]. Damit halten sich die elastischen Spannungen, die durch die unterschiedlichen Diffusionsgeschwindigkeiten und den dadurch bedingten Leerstellenstrom ausgelöst werden, in Grenzen. Sie wirken sich erst bei viel breiteren Diffusionsprofilen aus und führen zu einer Verschiebung der Schweißebene (Kirkendall-Effekt) und, falls dies aufgrund der Probengeometrie möglich ist, zu plastischer Relaxation an der Oberfläche. In der Literatur wurde daher der Einfluß des Leerstellenstroms bei hohen Temperaturen und einem Diffusionsprofil von mindestens einigen Mikrometern Breite untersucht.

Ein weiteres System, daß sich zur Untersuchung der Auswirkung von elastischen Spannungen auf die Diffusion eignet, ist Silber-Palladium. Das System ist über den gesamten Konzentrationsbereich mischbar, es sind keine intermetallischen Phasen bekannt [67]. Der Gitterparameter von Silber beträgt 0.40857 nm, der von Palladium $0.3890 \mathrm{~nm}$ [80]. Daraus ergibt sich eine moderate Gitterfehlpassung von $4.9 \%$. Die Diffusion wird merkbar durch stark unterschiedliche Diffusionsgeschwindigkeiten der beiden Elemente beeinflußt. Die partiellen Diffusionskoeffizienten unterscheiden sich um mehr als drei Dekaden $\left(D_{A g} / D_{P d} \geq 10^{3}\right)$, 


\section{Interdiffusion}

wodurch ein signifikanter Leerstellenstrom vom Palladium zum Silber entsteht. Im Unterschied zum System Kupfer-Gold fließt hier jedoch der Leerstellenstrom in Richtung der größeren Atomsorte. Im Fall einer epitaktischen Grenzfläche ändert sich damit die vom Leerstellenstrom auf die Versetzung ausgeübte Kraft. Sie treibt in diesem Fall die Versetzungen zu der ursprünglichen Grenzfläche zurück und behindert dadurch die Diffusion. In einer umfangreichen Untersuchung zur Interdiffusion in epitaktischen Silber-Palladium Schichten wurde genau dieses Verhalten gefunden $[52,53,54,55]$. Das Versetzungsnetzwerk, das zur Anpassung des Gitterparameters an der Grenzfläche eingebaut wird, verweilt trotz Interdiffusion in der Nähe der ursprünglichen Grenzfläche. Im Verlauf der Interdiffusion werden die Folgen der sich aufbauenden elastischen Spannungen sichtbar. Wie auch in dieser Arbeit wurde während der Interdiffusion eine diffusionsinduzierte Korngrenzwanderung beobachtet. Auch wurde ein heterogener Verlauf der Reaktion gefunden. Während in einigen Regionen die Reaktion schon lange begonnen hat, befinden sich andere Stellen quasi noch im Ausgangszustand. Neben diesen auch in dieser Arbeit beobachteten Prozessen wurde zusätzlich eine diffusionsinduzierte Rekristallisation beobachtet. Auch dieser Prozeß wird durch von der Diffusion verursachte Spannungen angetrieben. In Experimenten mit dünnen Palladium/Silber Schichten wurde eine Auflösung der Schichtstruktur zugunsten einer Kornstruktur mit silber- und palladiumreichen Körnern beobachtet [42,107,122]. Ein ähnliches Verhalten wird auch bei Kobalt/Silber Schichten gefunden [30].

Damit ergibt sich das folgende Gesamtbild: Wie in diesem Kapitel dargelegt können elastische Spannungen während der Interdiffusion einen großen Einfluß auf den Reaktionsverlauf haben. Speziell wenn es sich um epitaktische Schichtstrukturen handelt, kann es zu sehr komplexen Prozessen während der Interdiffusion kommen, da in diesem Fall nur die in die Grenzfläche eingebauten Versetzungen ein Leerstellenungleichgewicht ausgleichen können. Als ein wichtiger Parameter, der im Fall epitaktischer Schichten die strukturelle Entwicklung beeinflußt, stellt sich die Relation zwischen den Gitterparametern und den Diffusionsgeschwindigkeiten heraus. Ist, wie im Fall von Silber-Palladium, die schnellere auch die größere Komponente, so werden die Versetzungen an die ursprüngliche Grenzfläche zurückgetrieben. Im umgekehrten Fall, wie im System Kupfer-Gold, verteilen sich die Defekte über die gesamte Diffusionszone. Dieses Verhalten stimmt auch mit den qualitativen Überlegungen von Hirth [97] überein, die bei der Modellierung der Diffusion in epitaktischen Schichten unter dem Einfluß elastischer Spannungen gewonnen wurden. Auch zeigt sich, daß unter dem Einfluß elastischer Spannungen die Schichtstruktur aufgebrochen und eine neue Kornstruktur gebildet werden kann. Die Kinetik verursacht dabei Mischkonzentrationen, für die es nach dem Phasendiagramm zu keiner erhöhten Stabilität kommen sollte. In diesem Fall ist die Beschreibung mittels eines eindimensionalen Diffusionsmodells nicht mehr korrekt. Allgemein kann festgehalten werden, daß die Mikrostruktur der Schichten den Verlauf der Interdiffusion wesentlich beeinflußt. 


\section{Interreaktion in Kupfer/Gold}

Im folgenden Kapitel werden die gewonnen Erkenntnisse über die Interdiffusion in Kupfer/Gold Schichtsystemen auf die Phasenbildung angewendet. Dazu wurden Auslagerungstemperaturen unterhalb von $390{ }^{\circ} \mathrm{C}$, der Ordnungstemperatur von $\mathrm{Cu}_{3} \mathrm{Au}$ gewählt. Damit konnten die Phasen $\mathrm{CuAu}$ und $\mathrm{Cu}_{3} \mathrm{Au}$ gebildet werden. Die niedrigste untersuchte Auslagerungstemperatur betrug $230{ }^{\circ} \mathrm{C}$, bei der auch eine goldreiche Ordnungsphase auftreten könnte. Da die verschiedenen Reaktionsstadien sehr stark denen der Interdiffusion in Kupfer/Gold Schichtsystemen ähneln, wurde auf eine erneute, umfangreiche Darstellung verzichtet. Bei entsprechende Ähnlichkeiten wird auf die schon früher gezeigten Ergebnisse verwiesen.

\subsection{Experimentelle Ergebnisse}

Für eine makroskopische Charakterisierung wurden SNMS Messungen an einem bei $350{ }^{\circ} \mathrm{C}$ für verschiedene Zeiten ausgelagerten $\mathrm{Au} / \mathrm{Cu} / \mathrm{Au} / \mathrm{Cu} / \mathrm{Au}$ Schichtsystem auf einem Kupfereinkristall durchgeführt. Die Einzelschichtdicke betrug $60 \mathrm{~nm}$. Die SNMS Messungen, die in Abbildung 7.1 dargestellt sind, zeigen ein ähnlichen Verlauf, wie er schon bei der Untersuchung der Interdiffusion beobachtet wurde. In der ersten Phase der Reaktion findet man jedoch keinen sichtbaren Anstieg der Konzentration in der Goldschicht wie bei der Auslagerung bei $430{ }^{\circ} \mathrm{C}$. Trotzdem zeigt die elektronenmikroskopische Untersuchung der ausgelagerten Schichten bis zur tiefsten untersuchten Auslagerungstemperatur von $230{ }^{\circ} \mathrm{C}$ die gleiche Defektstruktur, die in der Frühphase der Interdiffusion gefunden wurde. Im Fall der bei $230{ }^{\circ} \mathrm{C}$ ausgelagerten Probe beträgt die Breite der Defektstruktur etwa 10 bis $15 \mathrm{~nm}$, was der Breite der Defektstruktur bei $430^{\circ} \mathrm{C}$

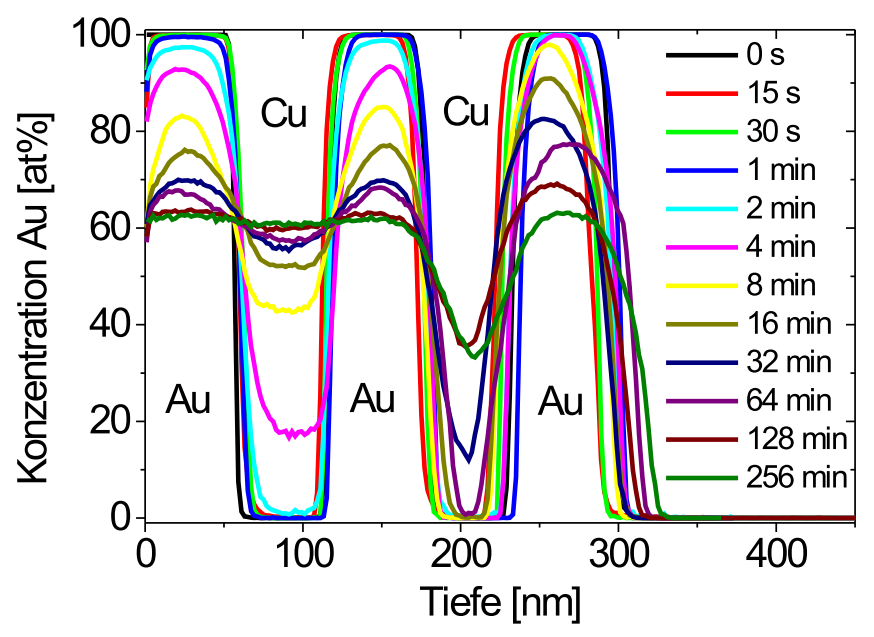

Abb. 7.1. Laser-SNMS Messung eines für verschiedene Zeiten bei $350{ }^{\circ} \mathrm{C}$ ausgelagerten $\mathrm{Au} / \mathrm{Cu} / \mathrm{Au} / \mathrm{Cu} / \mathrm{Au}$ Schichtpakets auf einem Kupfereinkristall. Nach einer Inkubationszeit von mehr als einer Minute beginnt die Reaktion in den obersten drei Schichten. Erst sehr viel später folgen auch die fünfte und dann die vierte Schicht. 


\section{Interreaktion in Kupfer/Gold}

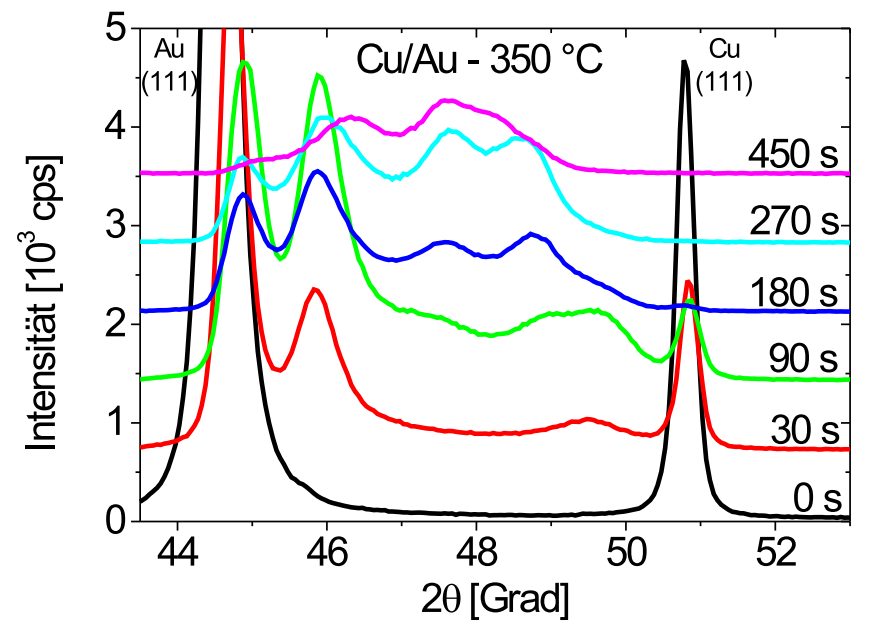

Abb. 7.2. Röntgenbeugungsuntersuchung einer bei $350{ }^{\circ} \mathrm{C}$ für verschiedene Zeiten ausgelagerten Kupfer/Gold-Schichtprobe mit einer Einzelschichtdicke von $60 \mathrm{~nm}$. Nach einer Zeit von 30 Sekunden treten erste neue Reflexe im Diffraktogramm auf. Nach der längsten untersuchten Auslagerungszeit von 450 Sekunden sind die beiden Elemente weitgehend vermischt.

entspricht. Die plattenförmigen Defekte verlaufen auch hier wieder unter einem flachen Winkel zur Grenzfläche. Aufgrund der gleichen Ausrichtung der Defekte und in der Probe vorhandenen Zwillinge kann direkt aus den Hellfeldaufnahmen auf die gleiche Defektstruktur auf den gemeinsamen (111) Ebenen der beiden Gitter geschlossen werden.

Nach einer Inkubationszeit von mehr als 1 Minute verändert sich bei der in Abbildung 7.1 dargestellten SNMS Messung die Konzentration in den drei obersten Schichten. Ein Vergleich mit elektronenmikroskopischen Untersuchungen zeigt, daß auch hier das Gefüge, wie bei der Untersuchung der Interdiffusion, in den Schichten neu gebildet wird. Eine qualitative Betrachtung der Grenzfläche ergibt jedoch einen viel heterogeneren Verlauf der Reaktion. Speziell bei der niedrigsten Auslagerungstemperatur von $230{ }^{\circ} \mathrm{C}$ findet man entweder eine über das Schichtpaket hinweg neugebildete Kornstruktur oder die von der Interdiffusion bekannte Defektstruktur an der ursprünglichen Grenzfläche vor. Wohlgemerkt findet man die beiden Zustände innerhalb einer Probe an weit auseinanderliegenden Stellen vor. Die Keimbildung ist bei den niedrigeren Temperaturen zunächst der Reaktionsschritt, der die Kinetik bestimmt. Da die Proben eine semikohärente Grenzfläche zwischen Kupfer und Gold aufweisen, kann man auf den gleichen Keimbildungsmechanismus wie bei den Interdiffusionsexperimenten schließen. Als Keimbildungszentren kommen somit ausgezeichnete Stellen wie zum Beispiel Tripelpunkte zwischen einer Grenzfläche und einer Kleinwinkelkorngrenze in der Schicht in Frage. Auch im Fall des Kupfersubstrats wächst die neue Kornstruktur langsam in das Substrat hinein.

Auch die Röntgenbeugungsuntersuchung, wie sie in Abbildung 7.2 dargestellt ist, zeigt große Ähnlichkeit mit der Interdiffusionsuntersuchung in Kupfer-Gold bei $430{ }^{\circ} \mathrm{C}$ aus Abbildung 6.9. Nach einer Auslagerung für 30 Sekunden bei $350{ }^{\circ} \mathrm{C}$ findet man im Röntgendiffraktogramm eine Kupferanreicherung der Goldschicht 


\section{Interreaktion in Kupfer/Gold}

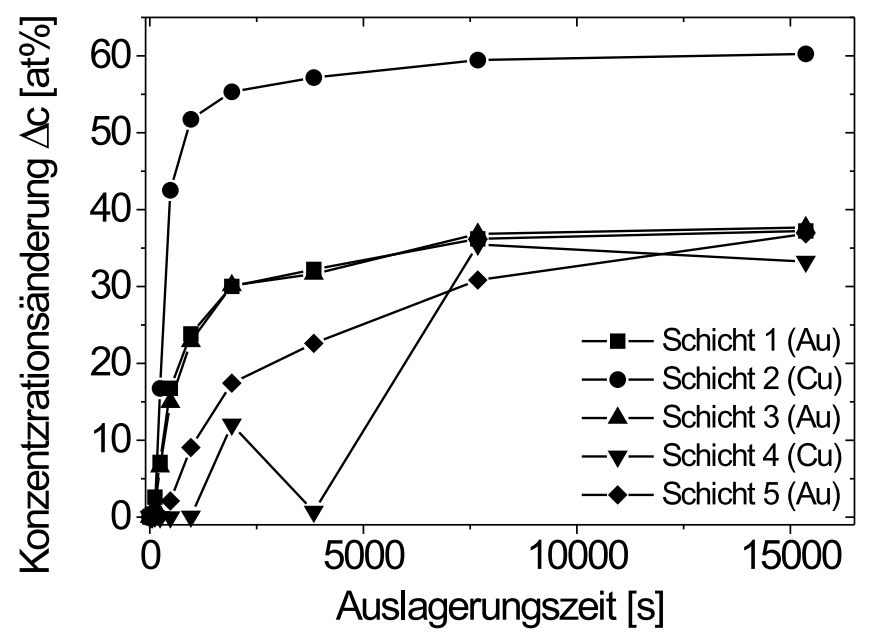

Abb. 7.3. Änderung der Peakkonzentration in den verschiedenen Schichten für die verschiedenen Auslagerungszeiten. Die 4. und 5. Schicht weichen deutlich vom Verhalten der darüber liegenden Schichten ab.

und zwei neue Maxima mit einer kupfer- und einer goldreichen Zusammensetzung. Die neuen Maxima können nach den Ergebnissen aus der Interdiffusion einem kupfer- und einem goldreichen Mischkristall zugeordnet werden. Im Verlauf der Auslagerung treten weitere Maxima auf. Jedoch kann kein Maximum des Röntgendiffraktogramms eindeutig einer Ordnungsphase zugeordnet werden. An der Position der korrespondierenden Ordnungsreflexe findet man in keinem Fall eine erhöhte Intensität. Damit wird während der Auslagerung noch keine langreichweitige Ordnung ausgebildet. Eine Nahordnung kann in der verwendeten Konfiguration von der Röntgenbeugung jedoch nicht erfaßt werden. Bei der Vielzahl der auftretenden Reflexe scheinen die Ordnungsstrukturen noch keine ausgezeichneten, im Vergleich zum Mischkristall stabilen Phasen zu bilden.

Bei den SNMS Messungen fällt die abweichende Kinetik der untersten Schichten auf. Ähnliches wurde auch schon bei der Untersuchung der Interdiffusion in dieser Arbeit gefunden. In Abbildung 7.3 wurde dazu die Anreicherung der Schichten mit der jeweils anderen Komponente aufgetragen. Während bei den obersten drei Schichten die Reaktion schnell beginnt und die Konzentration einen Gleichgewichtswert erreicht, beginnt die Reaktion in der untersten Kupfer- und Goldschicht erst viel später. Besonders fällt das Verhalten der vierten Kupferschicht auf, da sich die Anreicherung auch nach der längsten untersuchten Auslagerungszeit von 256 Minuten wesentlich von der zweiten Kupferschicht unterscheidet. Die Reaktion ist selbst auf der $78 \times 78 \mu \mathrm{m}^{2}$ großen Analysefläche der SNMS sehr inhomogen. Die Keimbildung ist damit, wie schon bei der Interdiffusion dargelegt, deutlich von den mechanischen und kinetischen Eigenschaften der Umgebung abhängig. Speziell die Keimbildung in der harten Kupferschicht wird mit zunehmender Schichtdicke schwieriger, da die bei der Keimbildung auftretenden Spannungen dort nicht mehr so gut über die Oberfläche relaxiert werden können. Bei den niedrigen Temperaturen beeinflußt die Keimbildung an der Grenzfläche 


\section{Interreaktion in Kupfer/Gold}

in den epitaktischen Schichtsystemen wesentlich den Reaktionsverlauf und führt zu dem gefundenen sehr inhomogenen Reaktionsverlauf.

\subsection{Ordnungseinstellung}

Wie aus den in Abbildung 7.2 dargestellten Röntgenbeugungsuntersuchungen hervorgeht, werden im Verlauf der Schichtreaktion verschiedene Körner mit Mischkonzentrationen, die nicht der Konzentration der Ordnungsphasen entsprechen, gebildet. Betrachtet man dagegen die in Abbildung 7.4 dargestellte Röntgenbeugungsuntersuchung einer anderen Probe mit einer Einzelschichtdicke von $30 \mathrm{~nm}$, die bei niedrigen Temperaturen ausgelagert wurde, so findet man aufgrund der veränderten Kinetik eine andere Verteilung der gebildeten Mischkonzentrationen. In diesem Fall würde eine direkte Interpretation der Meßdaten aus Röntgendiffraktometermessungen auf eine Bildung der $\mathrm{Cu}_{3} \mathrm{Au}$ und der $\mathrm{CuAu}$ Phase führen. Jedoch wurden auch in diesem Fall keine Überstrukturreflexe und die damit verbundene langreichweitige Ordnung gefunden. Man kann auch hinsichtlich der bei ähnlich niedriger Auslagerungstemperatur $\left(230{ }^{\circ} \mathrm{C}\right)$ durchgeführten Charakterisierung davon ausgehen, daß das Gefüge innerhalb der Schichten neu gebildet wurde. Nur wird dabei aufgrund der kinetischen Bedingungen ungefähr die Zusammensetzung der Ordnungsphase gebildet. Vergleicht man die Mischungsenthalpien mit dem Enthalpiegewinn durch die Ordnungseinstellung bei $320 \mathrm{~K}$, so erhält man im günstigsten Fall ein Viertel der Reaktionsenthalpie für die Bildung der stöchiometrischen Ordnungsphase [106]. Aus den durchgeführten DSC Messungen, die im letzten Kapitel vorgestellt wurden, ergab sich eine im Vergleich zur Einstellung der Ordnungsphase dreimal höhere Reaktionsenthalpie. Jedoch war

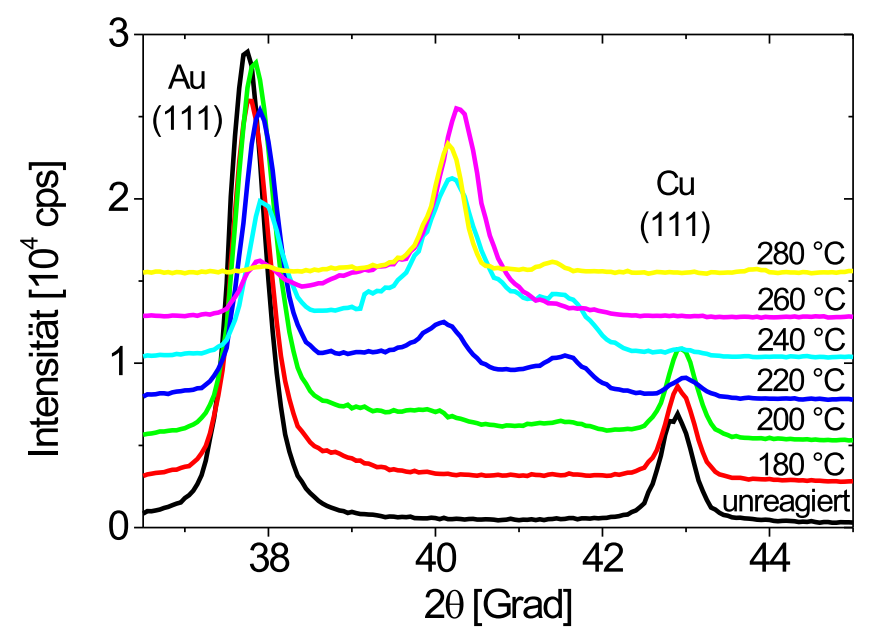

Abb. 7.4. Röntgendiffraktometermessung einer ausgelagerten Kupfer/Gold Schichtprobe mit einer Einzelschichtdicke von $30 \mathrm{~nm}$. Die Schicht wurde nacheinander bei den angegebenen Temperaturen für jeweils eine halbe Stunde ausgelagert. In keinem Auslagerungszustand sind Überstrukturreflexe sichtbar. Im Gegensatz zu den anderen Messungen wurde eine Kupferröntgenröhre verwendet. 


\section{Interreaktion in Kupfer/Gold}

Tab. 7.1. Zusammenstellung der Mischungsenthalpien aus den reinen Elementen und Ordnungsenthalpien bei einer Temperatur von $320 \mathrm{~K}$ aus [106].

\begin{tabular}{|c|c|}
\hline Prozess & Enthalpie $[\mathrm{J} / \mathrm{mol}]$ \\
\hline \hline Mischung $\mathrm{Cu}_{50} \mathrm{Au}_{50}$ & -6.98 \\
\hline Ordnung $\mathrm{CuAu}$ & -1.89 \\
\hline Mischung $\mathrm{Cu}_{75} \mathrm{Au}_{25}$ & -5.75 \\
\hline Ordnung $\mathrm{Cu}_{3} \mathrm{Au}$ & -1.32 \\
\hline
\end{tabular}

die Reaktion beim Erreichen der maximalen Temperatur beim ersten Aufheizen der Probe noch nicht abgeschlossen, so daß nicht die gesamte Reaktionsenthalpie gemessen werden konnte und so das kleinere Verhältnis der Enthalpien zu erklären ist. Bedenkt man, daß die gebildeten Körner von der stöchiometrischen Zusammensetzung der Ordnungsphasen abweichen und mit steigender Temperatur das Verhältnis noch ungünstiger wird, stammt fast der gesamte Enthalpiegewinn von der Durchmischung. Hier wird deutlich, daß es sich beim System Kupfer-Gold um ein schwach ordnendes System handelt. Eine Monte-Carlo-Simulation der Durchmischung und Ordnungseinstellung für das System Kupfer-Gold in einem einfachen Lagenmodell ergibt, daß nur eine Nahordnung im Gitter instantan bei geeigneter Konzentration eingestellt wird [93]. Betrachtet man die vergleichsweise geringe Bildungsenthalpie einer nichtstöchiometrischen Ordnungsstruktur, so wird verständlich, daß ein Korn mit einer bestehenden Ordnungsstruktur zugunsten eines anderen, energetisch günstigeren Mischungszustands in einem anderen Korn aufgelöst werden kann. Insgesamt zeigt sich, daß die Ordnungseinstellung nur einen sehr geringen Einfluß auf die Reaktionskinetik hat.

\subsection{Phasenbildungsmodelle}

In der Literatur findet man zwei klassische Modelltypen, die die Phasenauswahl bei der Interreaktion in dünnen Schichten beschreiben. Das Modell von Tu und Gösele [32] und dessen Erweiterungen [46] gehen von einer Lagenstruktur aus, wobei alle konkurrierenden Phasen schon vorhanden sein müssen. Die Keimbildung einer Phase wird nicht beschrieben. Aufgrund der Kinetik, die durch die Diffusion in den verschiedenen Phasen und die Transportbarrieren an den Grenzflächen bestimmt wird, ergibt sich die Wachstumsgeschwindigkeit der einzelnen Phasen. Aus diesen Wachstumsgeschwindigkeiten wird dann eine Phasensequenz und auch die Wachstumskinetik abgeleitet. Die Parameter, die die Grenzflächenbarriere beschreiben, sind jedoch schlecht zugänglich. Eine Beschreibung von realen Strukturen ist so nicht möglich. Da dieses Modell eine planare Schichtstrukur 


\section{Interreaktion in Kupfer/Gold}

bei der Phasenbildung annimmt, kann die Phasenbildung im Kupfer-Gold System damit grundsätzlich nicht beschrieben werden.

Die empirische Regel von Pretorius $[60,85,86]$ bezieht sich dagegen auf thermodynamische Größen. Nach ihr wird die Phase als erstes gebildet, die bei der Zusammensetzung der tiefsten Liquidustemperatur die größte effektive Bildungsenthalpie aufweist. Im Kupfer/Gold System ist dies die CuAu Phase, experimentell wird jedoch die $\mathrm{Cu}_{3} \mathrm{Au}$ Phase gefunden [113]. Pretorius erklärt die Abweichung von der im Experiment gefundenen $\mathrm{Cu}_{3} \mathrm{Au}$ Phase mit dem nur schwach ausgeprägten Minimum in der Liquiduslinie, das nur ungenau zu bestimmen ist, und mit den nur schwach ausgeprägten Ordnungsphasen.

Aus den vorangegangenen Untersuchungen ergibt sich ein anderes Bild für die Phasenbildung im Kupfer-Gold System. Aufgrund der Gitterfehlpassung und den daraus entstehenden Spannungen wird eine neue Kornstruktur gebildet. Eine bevorzugte Bildung von Körnern mit der stöchiometrischen Zusammensetzung der erwarteten Phasen ist nicht zu beobachten. Der Energiegewinn, der allein durch die Mischung der beiden Phasen entsteht, übersteigt den zusätzlich möglichen Energiegewinn durch die Ordnungseinstellung erheblich. Aufgrund der Kinetik werden zuerst Körner mit einer geringen Konzentration der jeweils anderen Komponente gebildet. So findet man bei einer Untersuchung der Phasensequenz die Bildung der kupferreichen $\mathrm{Cu}_{3} \mathrm{Au}$ und der goldreichen $\mathrm{CuAu}_{3}$ Phase vor der $\mathrm{CuAu}$ Phase.

\subsection{Diskussion}

In der Literatur wurde die Phasenbildung in dünnen Kupfer/Gold Schichten schon von mehreren Autoren untersucht und die scheinbar widersprüchlichen Ergebnisse teilweise kontrovers diskutiert. 1972 veröffentlichten Tu und Berry ihre mit der Röntgenbeugung bei einer Temperatur zwischen 160 und $220{ }^{\circ} \mathrm{C}$ gewonnenen Ergebnisse zur Schichtreaktion in Kupfer/Gold Schichten [113]. Nach ihren Untersuchungen wird eine $\mathrm{Cu}_{3} \mathrm{Au}$ Phase gefolgt von einer $\mathrm{CuAu}_{3}$ Phase gebildet. Die Bildung der $\mathrm{CuAu}$ Phase erfolgte sehr viel später. Die Identifikation der Phasen erfolgte über die zwischen den erlaubten Reflexen gebildeten Intensitätsmaxima. Eine Bildung von Überstrukturreflexen wird nicht berichtet. Unter Annahme einer planaren Schichtstruktur finden die Autoren eine lineare Verbreiterung der gebildeten Phase mit der Zeit. Als den die Kinetik bestimmenden Schritt wird der Einbau der Atome in die geordnete Struktur angenommen, woraus sich eine lineares Zeitgesetz ergibt. Borders dagegen findet in seinen RBS Messungen an Kupfer/Gold Schichten eine kontinuierliche Durchmischung an der Grenzfläche ohne einen Hinweis auf die Anwesenheit von Ordnungsphasen [9]. In Röntgenuntersuchungen kann er die Ergebnisse von Tu und Berry bestätigen. Er schlägt zur 


\section{Interreaktion in Kupfer/Gold}

Erklärung der Meßergebnisse ein Wachstum der Ordnungsphase von der Korngrenze in die bestehenden Körner hinein vor. Für die in beiden Untersuchungen gefundene Abnahme der Korngröße konnte jedoch keiner der Autoren eine Erklärung geben.

Die Ergebnisse dieser Arbeit führen zu einem anderen Bild. In einer ersten Phase wird bei einer epitaktischen Schicht die schon bei den Frühstadien der Interdiffusion gefundene Defektstruktur an der Grenzfläche gebildet. Als Folge wird in den Schichten ein neues Gefüge gebildet. Die Konzentration der neu gebildeten Körner hängt dabei von den kinetischen Verhältnissen während der Reaktion ab. Der Gleichgewichtszustand wird über eine Folge von Mischkonzentrationen eingestellt, die in manchen Fällen mit den Konzentrationen der Ordnungsphasen übereinstimmen. Die neu gebildete Kornstruktur besteht nach den elektronenmikroskopischen Untersuchungen aus kleineren Körnern als die ursprüngliche Schichtstruktur. Dies erklärt die bei der Interreaktion von Tu und Berry und Borders gefundene Abnahme der mittleren Konrgröße. Die Phasensequenz ergibt sich vollständig aus der Kinetik während der Interdiffusion.

Bei den genannten Artikeln wird jeweils auch eine Aktivierungsenergie für den Diffusionsprozess angegeben. Tu und Berry finden eine Aktivierungsenergie von $1.65 \mathrm{eV}$, Borders von 1.35 bis $1.5 \mathrm{eV}$. Beide Werte sind unter Annahme einer planaren Schichtstruktur ermittelt worden. Tu und Berry merken an, daß die Schichtdicke linear mit der Zeit wächst und nicht wie für ein diffusionskontollierten Prozess proportional zur Wurzel der Zeit ist. Deshalb wurde ein grenzflächenkontrollierter Prozess eingeführt, der die Aktivierungsenergie und das Wachstum der Schichtdicke besser beschreibt und aus dem der Wert für die Aktivierungsenergie gewonnen wurde. Allen Ansätzen gemeinsam ist die Annahme einer planaren Schichtstruktur, die jedoch nach den vorliegenden Ergebnissen nicht haltbar ist. Da die ermittelten Aktivierungsenergien aufgrund eines fehlerhaften Modells berechnet wurden, haben die Werte keine physikalische Bedeutung. Der im Rahmen dieser Arbeit ermittelte Wert für die Aktivierungsenergie von $1.18 \mathrm{eV}$ entspricht dem die Kinetik begrenzenden Prozess. Wie schon im letzten Kapitel wird dieser Wert mit der Korngrenzdiffusion verknüpft. 


\section{Zusammenfassung}

In dieser Arbeit wurde der Einfluß von Fehlpassungsspannungen auf die Interdiffusion und Interreaktion in Dünnschichtsystemen untersucht. Dazu wurden das System Kupfer-Gold und das System Silber-Gold als Beispiele für Systeme mit großer bzw. mit verschwindender Gitterfehlpassung im direkten Vergleich untersucht. Die im Rahmen dieser Arbeit hergestellten und charakterisierten epitaktischen Schichtsysteme mit einer Einzelschichtdicke um $100 \mathrm{~nm}$ wurden bei Temperaturen zwischen 230 und $430{ }^{\circ} \mathrm{C}$ ausgelagert. Die Interdiffusion wurde an Silber-Gold und an Kupfer-Gold oberhalb der höchsten Ordnungstemperatur von $410{ }^{\circ} \mathrm{C}$ untersucht. Die Interreaktion wurde im Bereich der Ordnungsphasen des Kupfer-Gold Systems studiert.

Zur Bearbeitung dieser Fragestellung sind chemische Analysetechniken mit hoher Ortsauflösung erforderlich. Dazu wurde die elektronenmikroskopische Z-Kontrastabbildung verwendet. Es wurde eine Beschreibung des Bildkontrasts abgeleitet und die Bedingungen, innerhalb derer eine quantitative Auswertung der Bildintensitäten möglich ist, bestimmt. Für die Sekundärneutralteilchenmassenspektroskopie mit Lasernachionisierung wurde ein Auswertungsverfahren angegeben, mit der eine quantitative chemische Analyse leicht möglich ist. Zusätzlich wurden auftretende Artefakte der Methoden beleuchtet.

Im Fall der Interdiffusion bei $350^{\circ} \mathrm{C}$ in epitaktischen Silber-Gold Schichten konnte keine Abweichung von einer normalen Korngrenz- und Volumendiffusion festgestellt werden.

Bei der Interdiffusion im Kupfer-Gold System mit epitaktischen Schichten findet man bei Interdiffusionsexperimenten oberhalb der höchsten Ordnungstemperatur von $430{ }^{\circ} \mathrm{C}$ einen zweistufigen Prozeß. In der ersten Phase werden die Defekte, die die Gitterfehlpassung zwischen den Schichten ausgleichen, durch die Volumendiffusion über die Diffusionszone verteilt. Während der Diffusion kommt es zu einer Verarmung an Leerstellen in der Diffusionszone, wodurch die Interdiffusion zum Stillstand kommt. In einer zweiten Phase werden neue Keime von Körnern neuer Orientierung an ausgezeichneten Punkten wie zum Beispiel an Tripelpunkten zwischen der Grenzfläche und einer durch die Schicht verlaufenden Kleinwinkelkorngrenze gebildet. Über die neu gebildeten Korngrenzen ist nun wieder ein schneller Transport und eine vereinfachte Bildung weiterer Körner möglich. Die Großwinkelkorngrenzen ermöglichen zudem eine schnellere Einstellung des Leerstellengleichgewichts. Im Vergleich zur hohen Reaktionsgeschwindigkeit kann über die Korngrenze nur eine geringe Zahl der jeweils anderen Komponente antransportiert werden. Dadurch weist die zuerst gebildete Kornstruktur nur eine geringe Konzentration von 15 bis 20 at\% der jeweils anderen Komponente auf. Im folgenden schreitet die Durchmischung mit der Bildung von höher angereicherten 


\section{Zusammenfassung}

Körnern und diffusionsinduzierter Korngrenzwanderung bis zur Einstellung einer Gleichgewichtskonzentration voran.

Die Reaktion in Kupfer/Gold Schichten unterhalb der Ordnungstemperatur ähnelt der der Interdiffusion oberhalb der Ordnungstemperatur sehr stark. Es treten die gleichen Reaktionsprozesse auf. Die möglichen Ordnungsphasen habe dabei aufgrund der im Vergleich zur Mischungsenthalpie geringen Ordnungsenthalpie keinen nachweisbaren Einfluß auf die Phasenbildung. Das Phasenbildungsmodell von Tu und Goesele [32] kann in diesem System aufgrund der falschen Voraussetzung planaren Schichtwachstums nicht angewendet werden. Auch die empirische Regel von Pretorius $[60,85,86]$ muß versagen, weil die Phasenbildung in Kupfer/Gold Schichtsystemen vollständig von der Kinetik der Interdiffusion bestimmt wird.

Der Spannungszustand in den Schichten beeinflußt die Gefügeneubildung stark. So wurde ein schnellerer Verlauf der Neubildung des Gefüges in oberflächennahen Bereichen, in denen Relaxationen über die Oberfläche möglich sind, gefunden.

Im Rahmen dieser Arbeit war eine quantitative Beschreibung der ersten Diffusionsphase aufgrund der Komplexität des Problems nicht möglich. Eine genauere chemische Analyse der Konzentrationsverteilung um die Defektstruktur, zum Beispiel mit Hilfe der dreidimensionalen Atomsonde, würde dieses Vorhaben erleichtern. Eine Beschreibung mit Hilfe von Computersimulationen aufgrund der dargestellten Ergebnisse ist jedoch in Vorbereitung [40]. 


\section{A. SNMS-Eichungen zum System Silber-Gold}

Wie auch für das System Cu-Au wurden TRIM-Simulationen des Abtragsverhaltens von Ag-Au unter Beschuß mit 700 eV Argon- und 25 keV Galliumionen durchgeführt. Die Ergebnisse sind in den folgenden Auftragungen zusammengefaßt. Für Ag-Au findet man ein Verhältnis zwischen der maximalen Silber- und Goldintensität von $1.91 \mathrm{zu} 1$.
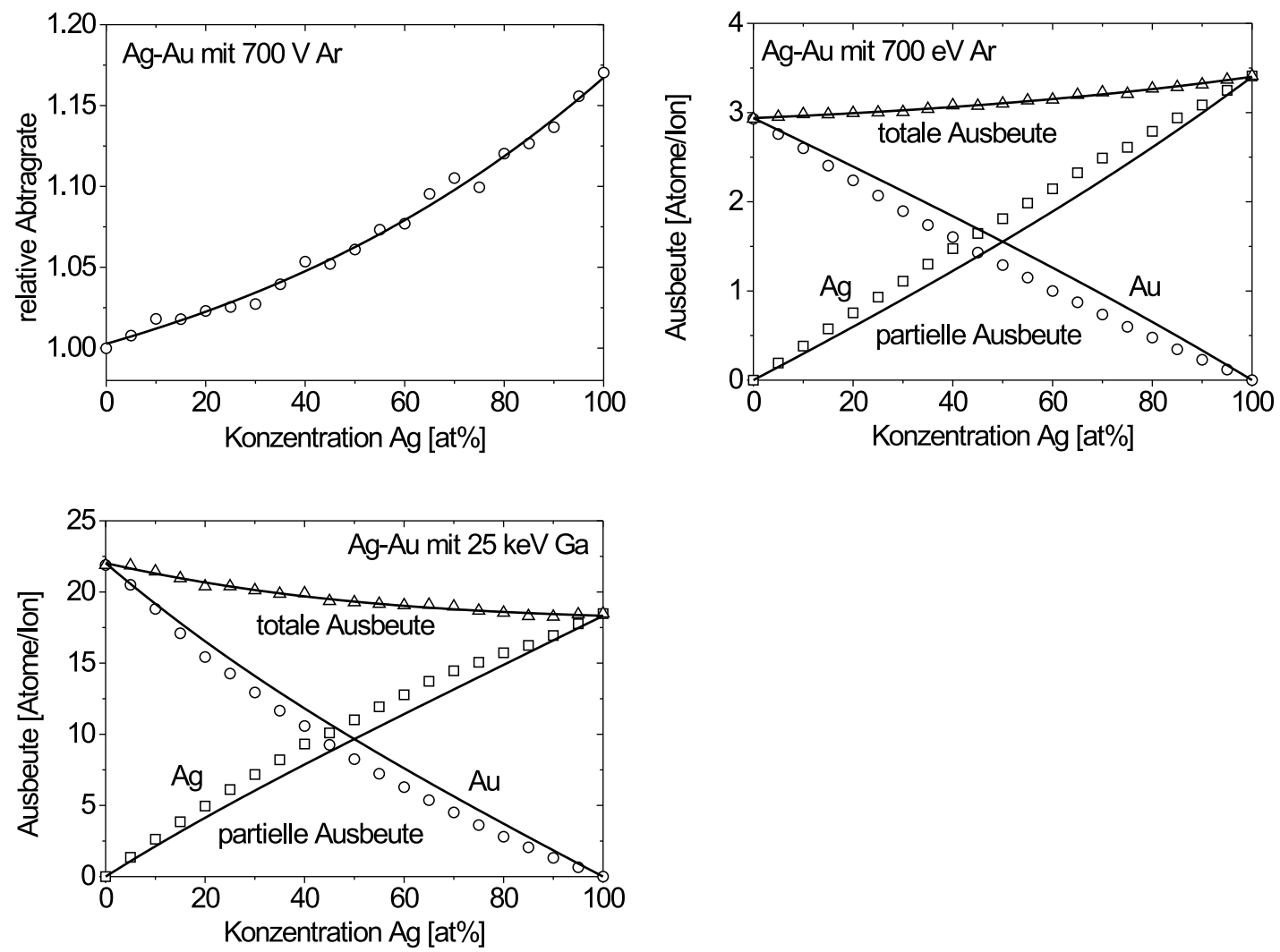

Abb. A.1. TRIM-Simulation im System Ag-Au mit $700 \mathrm{eV}$ Argon- und $25 \mathrm{keV}$ Galliumionen. 


\section{Literaturverzeichnis}

[1] A. N. Aleshin, V. K. Egorov, B. S. Bokstein und P. V. Kurkin, Thin solid films 223 (1993) 51.

[2] A. E. Austin, N. A. Richard und Van E. Wood, J. Appl. Phys. 37 (1966) 3651.

[3] M. Avrami, J. Chem. Phys. 7 (1939) 1103, 8 (1940) 212, 9 (1941) 177.

[4] C. A. B. Ball und J. H. van der Merwe, Phys. Status Solidi 38 (1970) 335.

[5] C. A. B. Ball, Phys. Status Solidi 42 (1970) 357.

[6] B. W. Batterman, J. Appl. Phys. 28 (1957) 556.

[7] G. Betz, Surface Sci. 92 (1980) 283.

[8] W. Bollmann, Crystal Defects and Crystalline Interfaces, Springer, New York (1970).

[9] J. A. Borders, Thin solid films 19 (1973) 359.

[10] R. Busch und V. Ruth, Acta metall. mater. 39 (1991) 1535.

[11] R. W. Cahn und P. Haasen (ed.), Physical Metallurgy, Amsterdam, Elsevier Science, 4. überarbeitete und erweiterte Auflage (1996).

[12] A. Chatterjee und D. J. Fabian, Acta Metall. 17 (1969) 1141.

[13] J. W. Christian, The theory of transformations in metals and alloys Pergamon Press, Oxford, 1. Auflage (1965).

[14] H. E. Cook und J. E. Hilliard, J. Appl. Phys. 40 (1969) 2191.

[15] J. M. Cowley, Phys. Rev. 77 (1950) 669.

[16] A. V. Crewe und J. Wall, Science 168 (1970) 1338.

[17] M. J. Dallwitz, Acta metall. 20 (1972) 1229.

[18] R. D. Doherty, Encyclopedia of Mater. Sci. and Eng., Supllementary Vol. 3, Ed. R. W. Cahn, Pergamon, Press, Oxford (1992), p. 1695.

[19] P. A. Doyle und P. S. Turner, Acta Cryst. A 24 (1968) 390.

[20] J. DuMond und J. P. Youtz, J. Appl. Phys. 11 (1940) 357. 


\section{Literaturverzeichnis}

[21] J. W. du Plessis und J. H. van der Merwe, Phil. Mag. 20 (1965) 1265.

[22] J. C. Ewert, F. Hartung und G. Schmitz, Appl. Phys. Lett. 71 (1997) 1311.

[23] W. Färber, G. Betz und P. Braun, Nucl. Instr. Methods 132 (1976) 351.

[24] J. C. Fisher, J. Appl. Phys. 22 (1951) 74.

[25] N. H. Fletcher, J. Appl. Phys. 35 (1964) 234.

[26] N. H. Fletcher, Phil. Mag. 16 (1966) 159.

[27] F. C. Frank und J. H. van der Merwe, Proc. R. Soc. London, Ser. A. 198 (1949) 205 und 216.

[28] J. Friedel, Phil. Mag. 46 (1955) 514.

[29] S. Fujikawa, M. Werner, H. Mehrer und A. Seeger, Mater. Sci. Forum 15-18 (1987) 431.

[30] E. E. Follerton, I. K. Schuller, R. Bhadra, M. Grimsditch und S. M. Hues, Mater. Sci. Eng. A 126 (1990) 197.

[31] G. Gladyszewski, Thin solid films 204 (1991) 473.

[32] K. Goesele und K. N. Tu, J. Appl. Phys. 53 (1982) 3252.

[33] S. Goudsmit und J. L. Saunderson, Phys. Rev. 57 (1940) 24.

[34] A. L. Greer, Defect and Diffusion Forum 129-130 (1996) 163.

[35] P. Haasen, Physikalische Metallkunde, Springer-Verlag, Berlin, 3. Auflage (1994).

[36] P. M. Hall, J. M. Morabito, N. T. Panousis, Thin solid films 41 (1977) 341.

[37] P. Hartel, H. Rose und C. Dinges, Ultramicroscopy 63 (1996) 63.

[38] F. Hartung, Untersuchung der Interreaktion von Cu-Au mit Hilfe der Z-Kontrast Elektronenmikroskopie, Diplomarbeit, Universität Göttingen (1996).

[39] F. Hartung, J. C. Ewert und G. Schmitz, Scripta Mat. 39 (1998) 79.

[40] F. Hartung und G. Schmitz, in Vorbereitung.

[41] J. P. Hirth und J. Lothe, Theory of dislocation, McGraw-Hill, New York, S. 458.

[42] M. Jalochowski und P. Mikolajczak, J. Phys. F: Met. Phys. 13 (1983) 1973. 


\section{Literaturverzeichnis}

[43] W. Jesser und D. Kuhlmann-Wilsdorf, Phys. Status Solidi 19 (1967) 95.

[44] W. A. Johnson, Trans. ASME 135 (1939) 1.

[45] W. A. Johnson, Trans. AIME 147 (1942) 331.

[46] W. C. Johnson und G. Martin, J. Appl. Phys. 68 (1990) 1252.

[47] W. Jost und K. Hauffe, Diffusion - Methoden der Messung und Auswertung, Dr. Dietrich Steinkopf Verlag, Darmstadt, 2. Auflage (1972).

[48] G. Kampwerth, M. Terhorst, E. Niehuis und A. Benninghoven, Proceedings of the Eighth International Conference on Secondary Ion Mass Spectroscopy (SIMS VII), ed. by A. Benninghoven, K. T. F. Janssen, J. Tümpner und H. W. Werner, John Wiley \& Sons, Chichester, 1992.

[49] I. Kaur und W. Gust, Fundamentals of grain und interphase boundary diffusion, Ziegler Press, Stuttgart, 2. überarbeitete Auflage (1989).

[50] H. E. Kissinger, Journal of Research of the National Bureau of Standsards 57 (1956) 217.

[51] H. E. Kissinger, Analyt. Chem. 29 (1957) 1702.

[52] V. M. Kosevich, A. N. Gladkikh, M. V. Karpovsky und V. N. Klimenko, Interface Science 2 (1994) 247.

[53] V. M. Kosevich, A. N. Gladkikh, M. V. Karpovsky und V. N. Klimenko, Interface Science $\mathbf{2}$ (1994) 261.

[54] V. M. Kosevich, A. N. Gladkikh, M. V. Karpovsky und V. N. Klimenko, Interface Science $\mathbf{2}$ (1994) 271.

[55] V. M. Kosevich, V. N. Klimenko, A. N. Gladkikh und M. V. Karpovsky, Interface Science $\mathbf{2}$ (1995) 151.

[56] J. L. Labar und J. Morgiel, Micron 29 (1998) 425.

[57] Landolt-Börnstein, Group 3, Volume 26, Diffusion in Solid Metals and Alloys, Springer-Verlag, Berlin (1990).

[58] Larché und Cahn, Acta. metall. 30 (1982) 1835.

[59] Larché und Cahn, Acta. metall. 33 (1985) 331.

[60] J. Li, J. W. Strane, S. W. Russell, S. Q. Hong, J. W. Mayer, T. K. Marais, C. C. Theron und R. Pretorius, J. Appl. Phys. 72 (1992) 2810. 


\section{Literaturverzeichnis}

[61] Z. C. Liau, J. W. Mayer, W. L. Brown und J. M. Poate, J. Appl. Phys. 49 (1978) 5295.

[62] Q. Ma und R. W. Balluffi, Acta metall. mater. 41 (1993) 133.

[63] Q. Ma und R. W. Balluffi, Acta metall. mater. 41 (1993) 143.

[64] J. E. Macur und R. W. Vook, Thin solid films 66 (1980) 311.

[65] J. E. Macur und R. W. Vook, Thin solid films 66 (1980) 371.

[66] A. B. Martin, R. D. Johnson und F. Asaro, J. Appl. Phys. 25 (1954) 364.

[67] T. B. Massalski (ed.), Binary alloy phase diagrams, Metals Park, ASME (1987).

[68] J. W. Matthews, Phil. Mag. 13 (1966) 1207.

[69] J. W. Matthews, IBM Res. Rep. R. C. 4266 (No. 19084) (1973).

[70] K. Meinel, M. Klaua und H. Bethge, Thin solid films 34 (1976) 157.

[71] E. S. K. Menon, P. Huang, M. Kraitchman, J. J. Hoyt, P. Chow und D. de Fontaine, J. Appl. Phys. 1993 (73) 142.

[72] S. C. Moss, J. Appl. Phys. 35 (1964) 3547.

[73] W. D. Nix, Met. Trans. A 20A (1989) 2217.

[74] N. Norman und B. E. Warren, J. Appl. Phys. 22 (1951) 483.

[75] S. Ogawa und D. Watanabe, J. Appl. Phys. 22 (1951) 1502.

[76] R. L. Orr, J. Luciat-Labry und R. Hultgren, Acta Metall. 8 (1960) 431.

[77] A. Ourmazd, F. H. Baumann, M. Bode und Y. Kim, Ultramicroscopy 34 (1990) 237.

[78] V. Ozolinš, C. Wolverton und A. Zunger, Phys. Rev. B 57 (1998) 4816.

[79] V. Ozolinš, C. Wolverton und A. Zunger, Phys. Rev. B 57 (1998) 6427.

[80] W. B. Pearson, A Handbook of Lattice Spacings and Structures of Metals and Alloys, Volume 1 (1957) und 2 (1967), London.

[81] S. J. Pennycook und D. E. Jesson, Phys. Rev. Lett. 64, (1990) 938.

[82] D. D. Perovic, C. J. Rossouw und A. Howie, Ultramicroscopy 52 (1993) 353. 


\section{Literaturverzeichnis}

[83] P. Pirouz, F. Ernst, Y. Ikuhara, Sol. State Phenomena 59-60 (1998) 51.

[84] J. M. Poate, W.L. Brown, R. Homer, W.M. Augustyniak, J. W. Mayer, K. N. Tu und W. F. van der Weg, Nucl. Instrum. Methods 132 (1976) 345.

[85] R. Pretorius, A. M. Vredenberg und F. W. Saris, J. Appl. Phys. 70 (1991) 3636.

[86] R. Pretorius, T. K. Marais, A. E. Muller und D. Knoesen, Mat. Res. Soc. Symp. Proc. 238 (1992) 485.

[87] G. Radi, Acta Cryst. 26 (1969) 41.

[88] R. W. Roberts, Acta Metall. 2 (1954) 597.

[89] A. Romano, J. Vanhellemont, H. Bender und J.R. Morante, Ultramicroscopy 31 (1989) 183.

[90] G. Schmitz, Hochauflösende Elektronenmikroskopie zur strukturellen und analytischen Untersuchung binärer Aluminiumlegierungen., Dissertation, Universität Göttingen (1994).

[91] G. Schmitz und F. Haider, Scripta Mat. 37 (1997) 1951.

[92] G. Schmitz, J. C. Ewert und F. Hartung, Ultramicroscopy 77 (1999) 49.

[93] G. Schmitz, persönliche Mitteilung (2000).

[94] A. Schnieders, R. Möllers, M. Terhorst, H.-G. Cramer, E. Niehuis und A. Benninghoven, J. Vac. Sci. Technol. B 14 (4) 2712.

[95] B. Schönfelder, J. Traube und G. Kastorz, Phys. Rev. B 45 (1992) 613.

[96] P. Schwander, C. Kisielowski, M. Seibt, F. H. Baumann, Y. Kim und A. Ourmazd, Phys. Rev. Lett. 71 (1993) 4150.

[97] K. Shinohara und J. P. Hirth, Phil. Mag. 27 (1973) 883.

[98] G. Simmons und H. Wang, Single Crystal elastic constant and calculated aggregate properties: A Handbook, The M. I. T. Press, Cambridge, Massachusetts und London, 2. Auflage (1971).

[99] F. Soria und J. L. Sacedón, Thin solid films 60 (1979) 113.

[100] P. A. Stadelmann, Ultramicroscopy 21 (1987) 131.

[101] A. Stefan, Sitzungsbericht Wiener Akad. Wissensch. II 68 (1873) 385.

[102] G. B. Stephenson, Acta. metall. 36 (1988) 2663. 


\section{Literaturverzeichnis}

[103] D. Stenkamp und W. Jäger, Ultramicroscopy 50 (1993) 321.

[104] D. W. Stevens und G. W. Powell, Met. Trans. A 8A (1977) 1531.

[105] B. Straumal, E. Rabkin, W. Gust und B. Predel, Acta metall. mater. 43 (1995) 1817.

[106] B. Sundmann, S. G. Fries und W. A. Oates, Z. Mteallkd. 90 (1999) 4.

[107] K. Temst, M. J. Van Bael, C. Van Haesendonck, Y. Bruynseraede, D. G. de Groot, K. Koemann und R. Griessen, Thin solid films 342 (1999) 174.

[108] M. Terhorst, Laserionisierung und Flugzeitmassenspektroskopie zerstäubter Neutralteilchen: Grundlagen, Geräteentwicklung und Anwendung in der Oberflächen- und Mikrobereichsanalytik, Dissertation, Universität Münster (1994)

[109] C. T. Tomizuka, Bull. Amer. Phys. Soc. 2 (1957) 123.

[110] H. G. Tompkins, J. Vac. Sci. Technol. 16 (1979) 778.

[111] M. M. J. Treacy und J. M. Gibson, Ultramicroscopy 52 (1993) 31.

[112] M. M. J. Treacy und J. M. Gibson, Ultramicroscopy 54 (1994) 93.

[113] K. N. Tu und B. S. Berry, J. Appl. Phys. 43 (1972) 3283.

[114] J. H. van der Merwe, J. Appl. Phys. 34 (1963) 117 und 123.

[115] J. H. van der Merwe, Treatise on Materials Science and Technology, Vol. 2, H. Hermann (ed.), New York, Academic Press (1973).

[116] R. W. Vook und C. T. Horng, Phil. Mag. 33 (1976) 843.

[117] Z. L. Wang, Ultramicroscopy 53 (1994) 73.

[118] M. Watanabe, Z. Horita und M. Nemeto, Ultramicroscopy 65 (1996) 187.

[119] G. Wentzel, Z. Phys. 40 (1927) 590.

[120] C. Wolverton, V. Ozolinš und A. Zunger, Phys. Rev. B 57 (1998) 4332.

[121] S. K. Wonnell, J. M. Delaye, M. Bibolé und Y. Limoge, J. Appl. Phys. 72 (1992) 5195.

[122] T. Yamaguci, H. Mizubayashi, Y. Yoshihara, W. Song, A. Yamaguchi und R. Yamamoto, J. Magn. Mater. 156 (1996) 279.

[123] F.-L. Yang, A- L- Greer und R. E. Somekh, Thin solid films 275 (1996) 258. 


\section{Literaturverzeichnis}

[124] D. N. Yoon, Ann. Rev. Mater. Sci. 19 (1989) 43.

[125] J. Ziegler, J. Biersack und V. Littmark, The stopping and range of ions in solids. Pergamon, New York (1985). 


\section{Dank}

Nun ist es an der Zeit, den vielen Menschen zu danken, die mir die Zeit in Göttingen zu einer sehr angenehmen gemacht haben.

An erster Stelle möchte ich Dr. Guido Schmitz danken. Die vielen Diskussionen mit ihm und Anregungen von ihm haben meiner Arbeit immer wieder neue Impulse und mir einen tieferen Einblick in die physikalischen Zusammenhänge gegeben. Auch in den letzten Monaten ist es ihm trotz seines Auslandsaufenthalts in Los Angeles gelungen, durch intensive Nutzung der elektronischen Medien den Fortgang meiner Arbeit zu unterstützen.

Herrn Prof. Dr. R. Kirchheim danke ich für sein stetiges Interesse und die Unterstützung meiner Arbeit. Seine Tür stand immer offen, um das ein oder andere Problem zu besprechen.

Ein besonderer Dank gilt Dr. Jörg Ewert. Die gute Zusammenarbeit und die vielen Diskussionen haben sehr viel Spaß gemacht. Auch Dr. Astrid Pundt möchte ich herzlich danken. Die vielen nicht nur wissenschaftlichen Gespräche werden mir in Zukunft sicher fehlen.

Herrn Dr. P. J. Wilbrandt bin ich besonders für den unermüdlichen Einsatz bei der Durchführung der SNMS Messungen dankbar. Ihm, Herrn M. Hahn und Frau Ph. Quan danke ich für den Einsatz in allen Dingen, die die Elektronenmikroskopie betrafen. Ein großer Dank geht an Herrn Dr. M. Seibt und Herrn Dr. E. Spiecker für die allzeitige Unterstützung bei meinen Arbeiten am CM 200. Jürgen Dzick und Peter Troche danke ich für die Herstellung meiner ersten Schichtpakete. Herrn J. Herbst danke ich für die unbürokratische und schnelle Hilfe bei der Herstellung der ablösbaren Schichtpakete. Den Mitgliedern der Werkstätten und den Laboranten danke ich für ihre Hilfe bei der Lösung der vielen handwerklichen Probleme. Auch allen anderen Personen, die mir mit experimentellen Hilfestellungen und Ratschlägen das Leben erleichtert haben, sei gedankt.

Ein spezieller Dank geht an Dr. Bert Böddecker, Dr. Jan Bohlen, Boye Färber, Tobias Jeske, Philipp Kesten, Dr. Ruth Lüke, Michael Maxelon, Torsten Nägel, Ole Hölck, Dr. Dirk Paulmann, Peter Scholz und die vielen anderen, die für ein angenehmes Klima innerhalb wie auch außerhalb des Instituts gesorgt haben. Speziell die ständigen Einrichtungen wie die morgentliche Kaffeerunde mit den Anekdoten von Herrn Dr. H. G. Brion und Herrn Dr. F. D. Wöhler habe ich sehr zu schätzten gelernt.

Ganz besonders bedanke ich mich bei meiner Freundin Iris. Ich bin gespannt, was die Zukunft uns als nächstes bringt. 


\title{
Lebenslauf
}

\section{Persönliche Daten}

$\begin{array}{ll}\text { Name: } & \text { Frank Hartung } \\ \text { Geburtsort: } & \text { Fulda } \\ \text { Geburtsdatum: } & \text { 06.11.1969 } \\ \text { Nationalität: } & \text { deutsch }\end{array}$

\section{Ausbildung}

\author{
Schulbildung \\ 1976-1980 Grundschule in Zierenberg \\ 1980-1986 Gesamtschule in Zierenberg \\ 1986-1989 Oberstufengymnasium Kassel-Oberzwehren \\ Ausbildung zum mathematisch-technischen Assistenten \\ 05/1989 Allgemeine Hochschulreife \\ Abschluß als staatlich geprüfter \\ mathematisch-technischer Assistent \\ Studium \\ 10/89 Diplom-Physik Studium an der Georg- \\ August-Universität zu Göttingen \\ 07/91 Vordiplomprüfung Physik \\ 12/94 bis 11/96 Diplomarbeit am Institut für \\ Metallphysik mit dem Thema \\ "Untersuchung der Interreaktion \\ von $\mathrm{Cu}-\mathrm{Au}$ mit Hilfe der \\ Z-Kontrast Elekronenmikroskopie" \\ 11/96 Diplomprüfung im Fach Physik \\ seit 11/96 Wissenschaftlicher Mitarbeiter an der \\ Georg-August-Universität zu Göttingen \\ am Institut für Materialphysik \\ (vormals Inst. für Metallphysik) in der \\ Arbeitsgruppe von Prof. Dr. R. Kirchheim.
}

